\title{
Impact of Architectural Asymmetry on Frank-Kasper Phase Formation in Block Polymer Melts
}

\author{
Alice B. Chang, ${ }^{* \dagger}$ Frank S. Bates ${ }^{* \dagger}$ \\ ${ }^{\dagger}$ Department of Chemical Engineering and Materials Science, University of Minnesota, Minneapolis, MN 55455, United \\ States
}

SUPPORTING INFORMATION

Table of Contents

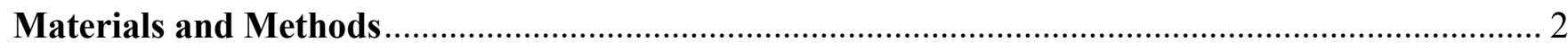

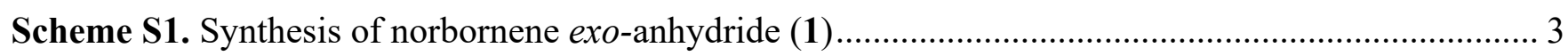

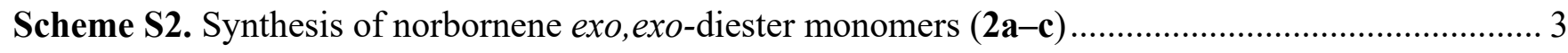

Scheme S3. Synthesis of norbornene exo- $n$-decylimide monomer (3) .............................................. 4

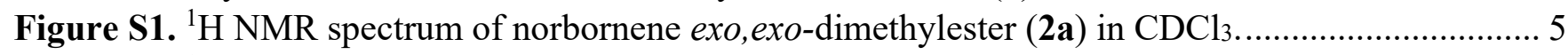

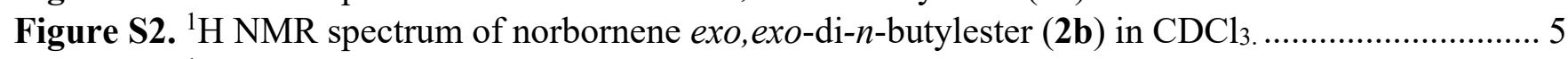

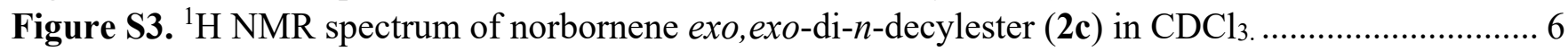

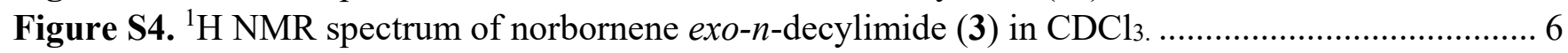

Figure S5. Representative synthesis of diblock polymers by ROMP .............................................. 7

Figure S6. Schematic of synthesis of block polymers using stock solutions.................................... 8

Figure S7. Superimposed ${ }^{1} \mathrm{H}$ NMR spectra comparing N2M/N2B and PN2M/PN2B ........................ 9

Figure S8. Superimposed ${ }^{1} \mathrm{H}$ NMR spectra comparing N2M/N1D and PN2M/PN1D ...................... 10

Figure S9. Superimposed ${ }^{1} \mathrm{H}$ NMR spectra comparing N2M/N2D and PN2M/PN2D ...................... 11

Figure S10. Representative SEC traces for ROMP block polymer synthesis.................................. 12

Figure S11. Comparison of SEC traces before and after SAXS heating experiments......................... 12

Figure S12. Comparison of ${ }^{1} \mathrm{H}$ NMR data before and after SAXS heating experiments ...................... 13

Table S1. Molecular characterization data for PN2M- $b$-PN2B block polymers. ............................... 14

Table S2. Molecular characterization data for PN2M- $b$-PN1D block polymers ................................ 15

Table S3. Molecular characterization data for PN2M- $b$-PN2D block polymers ................................ 16

Figure S13. DSC data for a PN2M- $b$-PN2D block polymer......................................................... 17

Table S4. Morphologies assigned for each pre-annealed $\mathrm{PN} 2 \mathrm{~B}\left(f_{\mathrm{PN} 2 \mathrm{M}}, N_{\mathrm{bb}}\right)$ block polymer $\ldots \ldots \ldots \ldots \ldots \ldots . . . . . . .18$

Table S5. Morphologies assigned for each pre-annealed PN1D $\left(f_{\mathrm{PN} 2 \mathrm{M}}, N_{\mathrm{bb}}\right)$ block polymer. ................. 19

Table S6. Morphologies assigned for each pre-annealed PN2D $\left(f_{\mathrm{PN} 2 \mathrm{M}}, N_{\mathrm{bb}}\right)$ block polymer................. 20

Table S7. Morphologies assigned for each unannealed PN2D $\left(f_{\mathrm{PN} 2 \mathrm{M}}, N_{\mathrm{bb}}\right)$ block polymer..................... 20

Figure S14. 1D averaged SAXS patterns for pre-annealed PN2M-b-PN2B block polymers................. 22

Figure S15. 1D averaged SAXS patterns for pre-annealed PN2M-b-PN1D block polymers ............... 26

Figure S16. 1D averaged SAXS patterns for pre-annealed PN2M-b-PN2D block polymers. ............... 29

Figure S17. 1D averaged SAXS patterns for unannealed PN2M-b-PN2B block polymers .................. 32 
Figure S18. 1D averaged SAXS pattern for C11. PN2D $(0.22,52)$ indexed to the FK $\sigma$ phase ............. 41

Figure S19. 1D averaged SAXS pattern for C14. PN2D $(0.26,46)$ indexed to the FK A15 phase ......... 42

Figure S20. 1D averaged MAXS/WAXS data for PN2M- $b$-PN2B ............................................. 43

Figure S21. 1D averaged MAXS/WAXS data for PN2M- $b$-PN1D ................................................. 44

Figure S22. Schematic illustration of side chain lengths estimated from backbone distances .............. 45

Estimation of statistical segment lengths by oscillatory shear rheology ................................... 45

Figure S23. van Gurp-Palmen plots for the homopolymers PN2M, PN2B, PN1D, and PN2D ........... 47

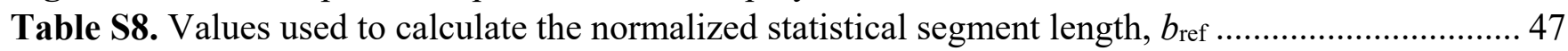

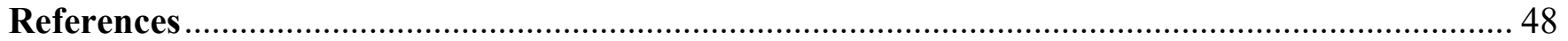

\section{Materials and Methods}

\section{Materials}

All chemicals were used as received unless otherwise noted. The second-generation Grubbs catalyst, $\left[\left(\mathrm{H}_{2} \mathrm{IMes}\right)\left(\mathrm{PCy}_{3}\right) \mathrm{Cl}_{2} \mathrm{Ru}=\mathrm{CHPh}\right]$ was purchased from Millipore Sigma, and the third-generation Grubbs catalyst (G3) was prepared according to a reported procedure. ${ }^{1}$ Norbornene endo-anhydride was purchased from TCI Chemicals and converted to the exo isomer (1). Benzene, methanol, $n$-butanol, $n$ decanol, trimethylamine, toluene, ethyl acetate, and hexanes were purchased from Millipore Sigma. Dichloromethane for ring-opening metathesis polymerization reactions was obtained from a solvent purification system and used in a glovebox. Deuterated chloroform for nuclear magnetic resonance (NMR) measurements was obtained from Cambridge Isotopes. Hermetic aluminum pans for differential scanning calorimetry and small-angle X-ray scattering measurements were purchased from DSC Consumables, Inc.

\section{Instrumentation}

${ }^{1} \mathrm{H}$ NMR

${ }^{1} \mathrm{H}$ NMR spectra were recorded on a $400 \mathrm{MHz}$ or $500 \mathrm{MHz}$ Bruker instrument. Deuterated chloroform was used as the solvent, and chemical shifts are reported in parts per million using residual protonated solvent as an internal standard $\left(\mathrm{CHCl}_{3}, 7.26 \mathrm{ppm}\right)$.

\section{Size-Exclusion Chromatography (SEC)}

SEC data were obtained using two Agilent PLgel MIXED-B $300 \times 7.5 \mathrm{~mm}$ columns with $10 \mu \mathrm{m}$ beads, connected to an Agilent 1260 Series pump, a Wyatt 18-angle DAWN HELEOS light scattering detector, and Wyatt Optilab differential refractive index detector. Online determination of $\mathrm{d} n / \mathrm{d} c$ assumed 100\% mass elution under the peak of interest. The mobile phase was tetrahydrofuran (THF).

\section{Small-Angle X-Ray Scattering (SAXS)}

SAXS data were collected at Beamline 12-ID-B at Argonne National Laboratory's Advanced Photon Source. The samples were studied using $13.3 \mathrm{keV}(0.932 \AA)$ X-rays and a sample-to-detector distance of $3.6 \mathrm{~m}$. The sample-to-detector distance was calibrated using a silver behenate standard. The beam was collimated using two sets of slits and a pinhole was used to removed parasitic scattering. The beam width was approximately 200-300 $\mu \mathrm{m}$ horizontally and 50 $\mu \mathrm{m}$ vertically. All samples were sealed under argon in hermetic aluminum DSC pans, and exposure times between 0.1 and $1 \mathrm{~s}$ were used for all measurements in order to minimize sample decomposition. The raw SAXS data was reduced and indexed using MATLAB software written by Dr. Byeongdu Lee (Argonne National Laboratory). 
Oscillatory Shear Rheology

Rheology data were collected on a Rheometric Scientific ARES rheometer using either $8 \mathrm{~mm}$ parallel plates (poly(norbornene exo,exo-dimethylester), PN2M) or $25 \mathrm{~mm}$ parallel plates (poly(norbornene exo,exo-di- $n$-butylester), PN2B; poly(norbornene exo-n-decylimide), PN1D; poly(norbornene exo,exo-di$n$-decylester), PN2D). Strain sweeps were performed in order to identify the linear viscoelastic regime. Applied strains were between 1 and $10 \%$ for all samples. Frequency sweeps were performed in the linear viscoelastic regime.

Differential Scanning Calorimetry (DSC)

DSC measurements were performed on a TA Q1000 under a dry nitrogen atmosphere. . The samples were heated to $150{ }^{\circ} \mathrm{C}$ at $10^{\circ} \mathrm{C} / \mathrm{min}$ to erase the thermal history, then cooled to $-100^{\circ} \mathrm{C}$ at $10^{\circ} \mathrm{C} / \mathrm{min}$. Data are reported here correspond to the second heating curve at $10{ }^{\circ} \mathrm{C} / \mathrm{min}$.

\section{Monomer Synthesis}

Scheme S1. Synthesis of norbornene exo-anhydride (1)

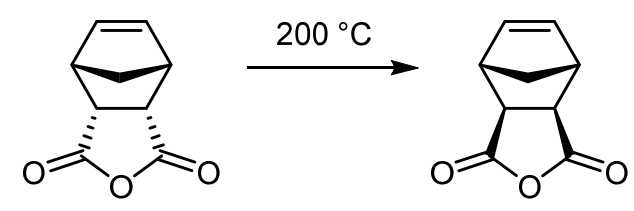

(1)

Norbornene exo-anhydride (1) was prepared according to a reported procedure. ${ }^{2} 200 \mathrm{~g}$ of commercially available norbornene endo-anhydride was added to a $500 \mathrm{~mL}$ round bottom flask equipped with a stir bar. The flask was fitted with a reflux condenser and heated to $200{ }^{\circ} \mathrm{C}$ for $12 \mathrm{hr}$ using a heating mantle. After $12 \mathrm{hr}$, the flask was removed from the mantle and allowed to slowly cool. When the contents of the flask reached approximately $70{ }^{\circ} \mathrm{C}, 50 \mathrm{~mL}$ benzene were added. The mixture was heated to reflux, and additional benzene was added to completely dissolve the contents of the flask. The mixture was allowed to slowly cool to room temperature, then the dark yellow mother liquor was decanted from the crystals. The contents of the flask were recrystallized seven times from benzene to afford pure exo-isomer 1 .

${ }^{1} \mathrm{H}$ NMR $\left(\mathrm{CDCl}_{3}\right): \delta 6.33(t, 2 \mathrm{H}), 3.45(s, 2 \mathrm{H}), 3.00(s, 2 \mathrm{H}), 1.65(m, 1 \mathrm{H}), 1.45(m, 1 \mathrm{H})$.

Scheme S2. Synthesis of norbornene exo,exo-diester monomers (2a-c)

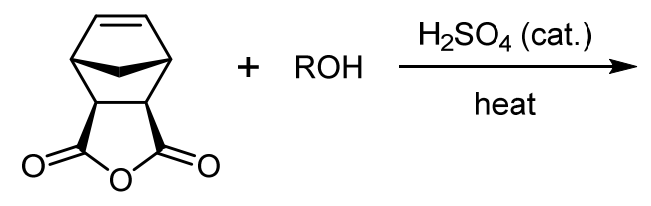

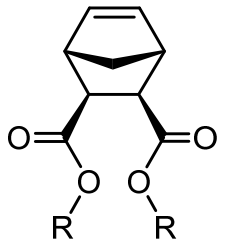

(2a-c)
$\mathrm{R}=\mathrm{Me}(\mathbf{2} \mathbf{a})$

$n-\mathrm{Bu}(\mathbf{2 b})$

$n$-Dec (2c)

Norbornene exo,exo-diester monomers were synthesized according to a reported procedure. ${ }^{3} \mathrm{In}$ a $100 \mathrm{~mL}$ round bottom flask equipped with a stir bar, norbornene exo-anhydride (1), 5 drops of concentrated $\mathrm{H}_{2} \mathrm{SO}_{4}$, and excess alcohol $\mathrm{ROH}$ were mixed (>10 equiv). The flask was sparged with nitrogen, then placed in an 
oil bath and heated for $12 \mathrm{hr}$. (The temperatures varied for different alcohols: $\mathbf{2 a}, 65{ }^{\circ} \mathrm{C} ; \mathbf{2 b}, 75{ }^{\circ} \mathrm{C} ; \mathbf{2 c}$, $90{ }^{\circ} \mathrm{C}$.) After $12 \mathrm{hr}$, the contents of each flask were concentrated in vacuo then dissolved in $\mathrm{CH}_{3} \mathrm{Cl}_{2}$. The solution was washed successively with saturated aqueous $\mathrm{NaHCO}_{3}$ and brine. The organic layer was dried over MgSo4, filtered, and concentrated in vacuo. The resulting colorless oil was purified: pure 2 a was obtained as white crystals by dropwise precipitation into stirring cold hexanes $\left(-78^{\circ} \mathrm{C}\right)$, and pure $\mathbf{2 b}$ and 2c were obtained as colorless oils by column chromatography with 1:4 ethyl acetate:hexanes. ${ }^{1} \mathrm{H}$ NMR spectra in $\mathrm{CDCl}_{3}$ are provided below (Figures $\mathrm{S} 1-3$ ).

- 2a: ${ }^{1} \mathrm{H}$ NMR $\left(\mathrm{CDCl}_{3}\right): \delta 6.21(t, 2 \mathrm{H}), 3.65(s, 6 \mathrm{H}), 3.09(m, 2 \mathrm{H}), 2.62(d, 2 \mathrm{H}), 2.10-2.12(m, 1 \mathrm{H})$, $1.49-1.51(m, 1 \mathrm{H})$.

- 2b: ${ }^{1} \mathrm{H}$ NMR $\left(\mathrm{CDCl}_{3}\right): \delta 6.20(t, 2 \mathrm{H}), 3.94-4.12(m, 4 \mathrm{H}), 3.07(m, 2 \mathrm{H}), 2.60(d, 2 \mathrm{H}), 2.12-2.14$ $(m, 1 \mathrm{H}), 1.54-1.63(m, 4 \mathrm{H}) 1.45-1.51(m, 1 \mathrm{H}), 1.31-1.43(m, 4 \mathrm{H}), 0.93(t, 6 \mathrm{H})$.

- 2c: ${ }^{1} \mathrm{H} \mathrm{NMR}\left(\mathrm{CDCl}_{3}\right): \delta 6.20(t, 2 \mathrm{H}), 3.92-4.11(m, 4 \mathrm{H}), 3.08(m, 2 \mathrm{H}), 2.60(d, 2 \mathrm{H}), 2.08-2.18(m$, $1 \mathrm{H}), 1.68-1.52(m, 4 \mathrm{H}) 1.45-1.51(m, 1 \mathrm{H}), 1.17-1.40(m, 30 \mathrm{H}), 0.88(t, 6 \mathrm{H})$.

Scheme S3. Synthesis of norbornene exo- $n$-decylimide monomer (3)
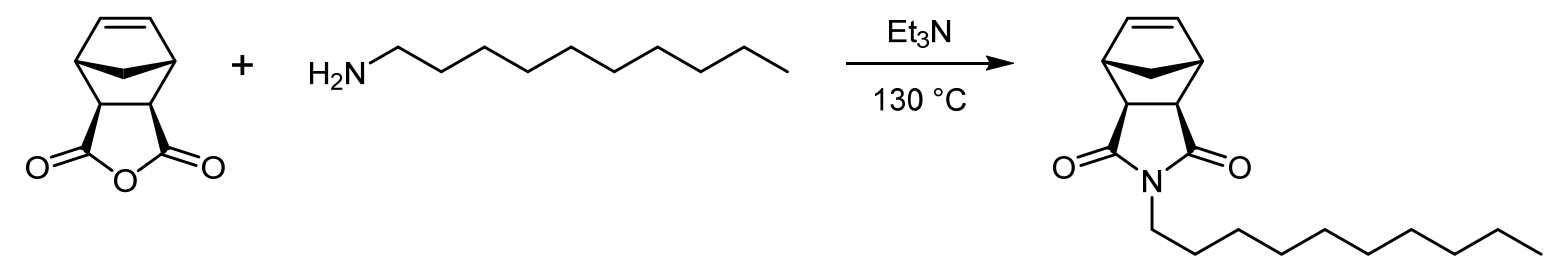

(3)

Norbornene exo-anhydride (1) (1.00 equiv., $5.00 \mathrm{~g}, 30.5 \mathrm{mmol}), n$-decylamine (1.05 equiv., $6.40 \mathrm{~mL}, 32.0$ mmol), triethylamine $(0.10$ equiv, $0.43 \mathrm{~mL}, 3.1 \mathrm{mmol})$, and toluene $(35 \mathrm{~mL})$ were added to a $100 \mathrm{~mL}$ round bottom flask equipped with a stir bar and a Dean-Stark trap. The mixture was heated to reflux for 12 hours. After 12 hours, the orange solution was cooled to room temperature, and toluene was removed in vacuo. The contents of the flask were dissolved in $\mathrm{CH}_{2} \mathrm{Cl}_{2}$, then washed successively with $0.1 \mathrm{~N}$ aqueous $\mathrm{HCl}$ and brine. The organic layer was dried over $\mathrm{MgSO}_{4}$, filtered, and concentrated in vacuo. The resulting slightly yellow oil was purified by column chromatography with 1:4 ethyl acetate:hexanes, affording pure 3 as a colorless oil. The ${ }^{1} \mathrm{H}$ NMR spectrum in $\mathrm{CDCl}_{3}$ is provided below (Figure S4).

${ }^{1} \mathrm{H}$ NMR $\left(\mathrm{CDCl}_{3}\right): \delta 6.28(t, 2 \mathrm{H}), 3.40-3.49(m, 2 \mathrm{H}), 3.27(m, 2 \mathrm{H}), 2.66(d, 2 \mathrm{H}), 1.46-1.58(m, 3 \mathrm{H}), 1.17-$ $1.33(m, 15 \mathrm{H}), 0.87(t, 3 \mathrm{H})$. 


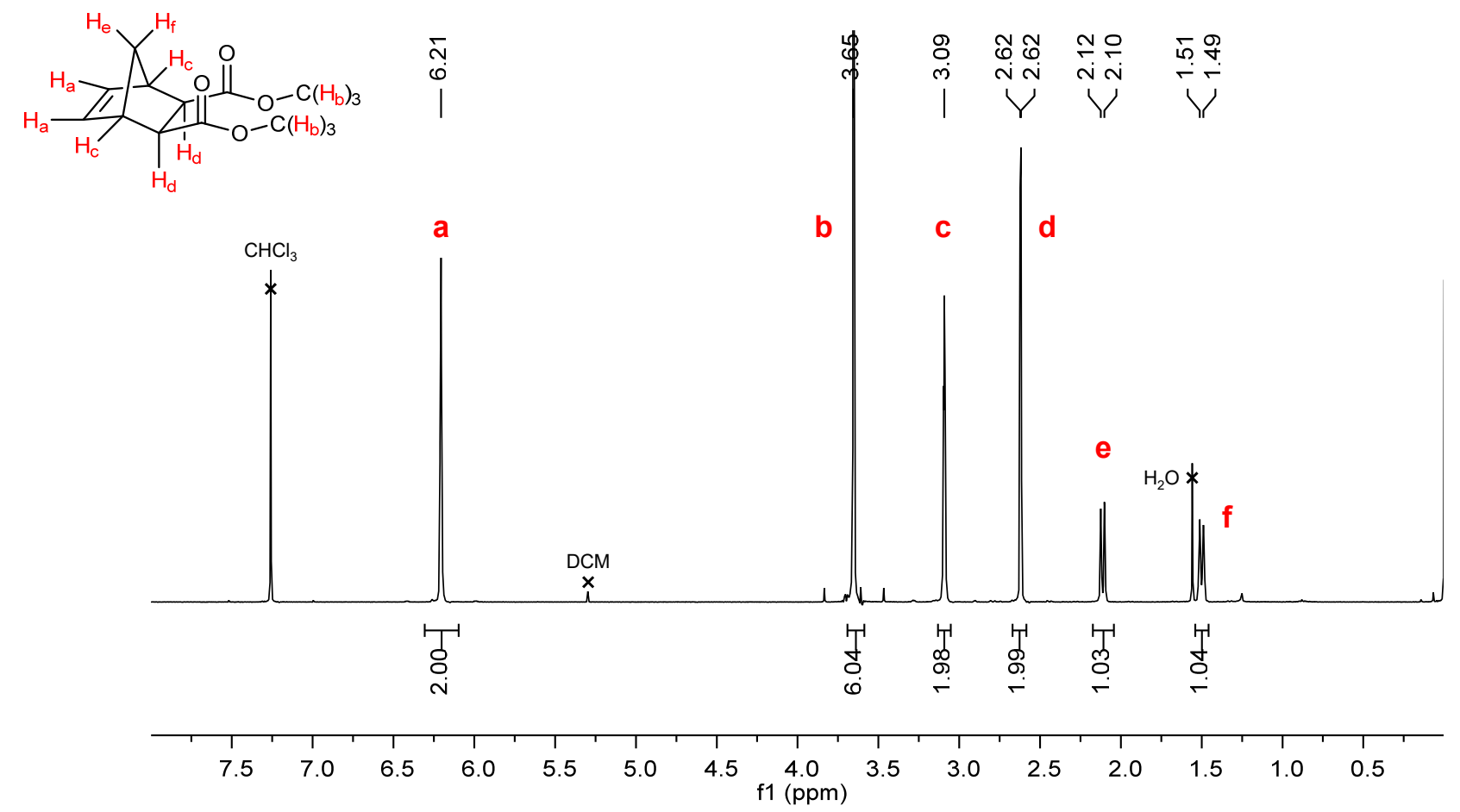

Figure S1. ${ }^{1} \mathrm{H}$ NMR spectrum of norbornene exo,exo-dimethylester (2a) in $\mathrm{CDCl}_{3}$.

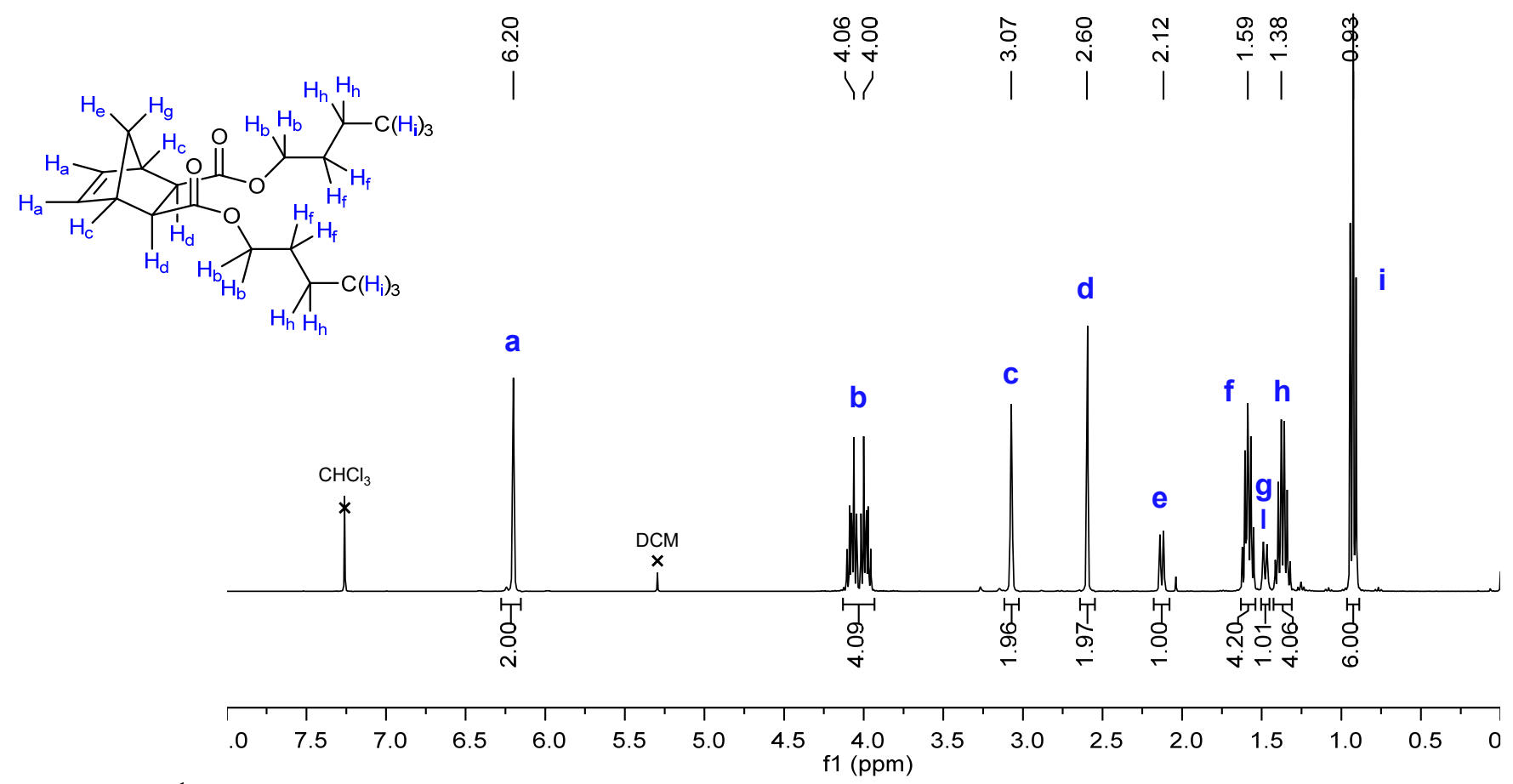

Figure S2. ${ }^{1} \mathrm{H}$ NMR spectrum of norbornene exo,exo-di- $n$-butylester (2b) in $\mathrm{CDCl}_{3}$. 


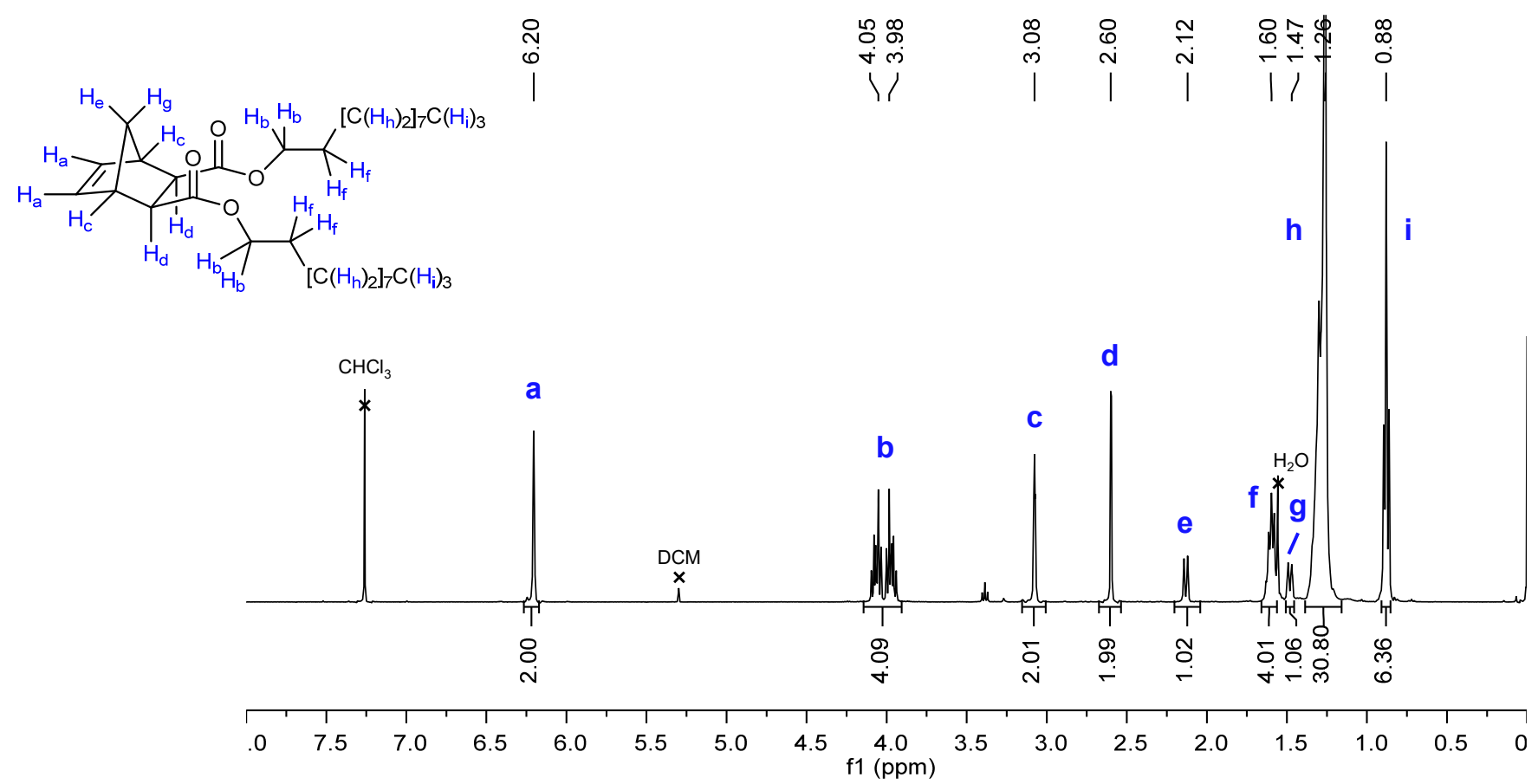

Figure S3. ${ }^{1} \mathrm{H}$ NMR spectrum of norbornene exo,exo-di- $n$-decylester $(\mathbf{2 c})$ in $\mathrm{CDCl}_{3}$.

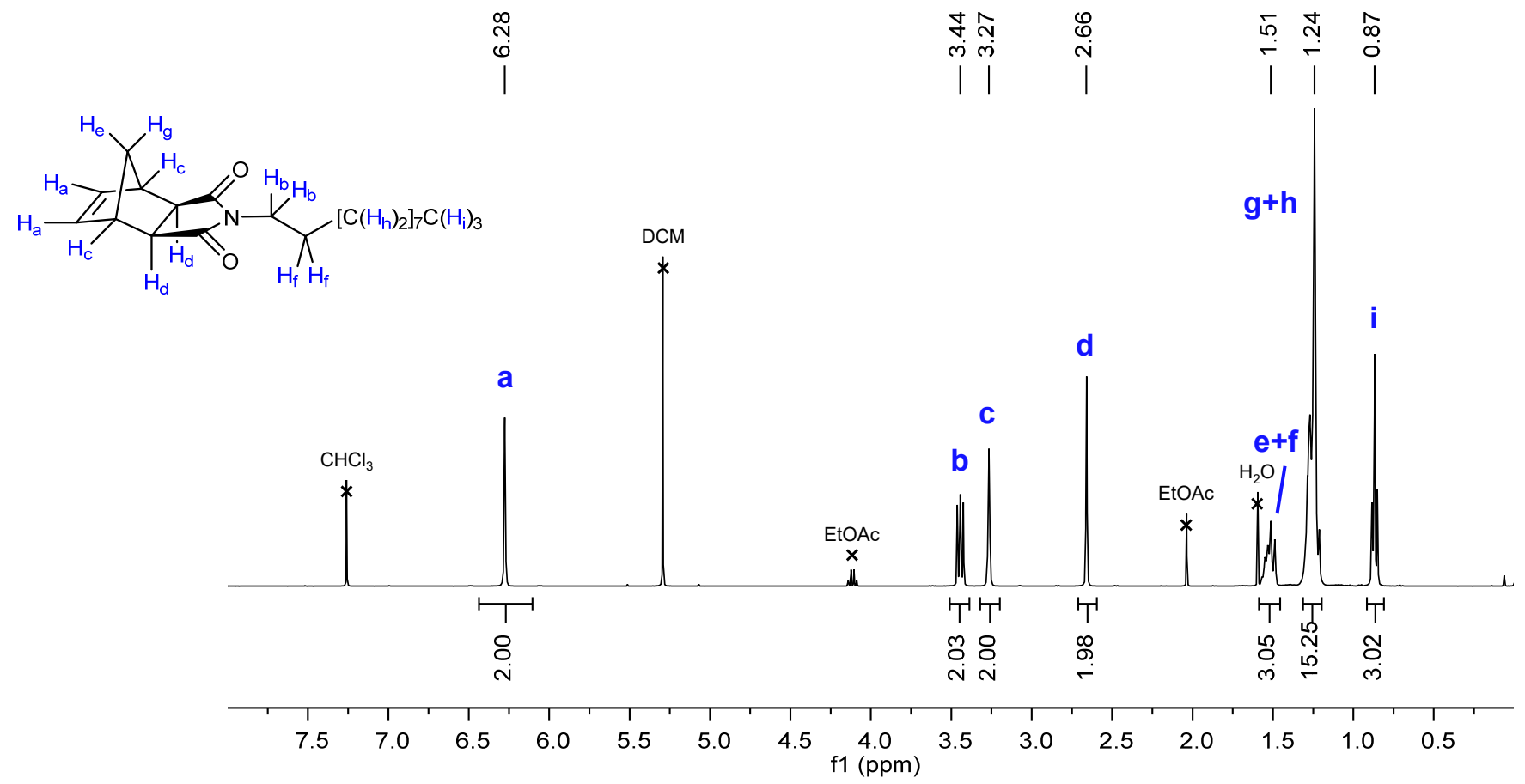

Figure S4. ${ }^{1} \mathrm{H}$ NMR spectrum of norbornene exo- $n$-decylimide (3) in $\mathrm{CDCl}_{3}$. 


\section{Block Polymer Synthesis}

Diblock polymers were synthesized by the sequential ring-opening metathesis polymerization (ROMP) of norbornenyl monomers. In a glovebox under inert argon atmosphere, stock solutions of each monomer was prepared in dichloromethane $(0.12 \mathrm{M})$. A stock solution of the third-generation Grubbs metathesis catalyst, $\left[\left(\mathrm{H}_{2} \mathrm{IMes}\right)(\mathrm{pyr})_{2}(\mathrm{Cl})_{2} \mathrm{Ru}=\mathrm{CHPh}\right](\mathbf{G 3})$, was also prepared in dichloromethane $(0.09 \mathrm{M})$. Stock solutions were used in order to facilitate fine variations in block lengths. The degree of polymerization of each block was determined by the number of molar equivalents of each monomer relative to 1 equivalent of the $\mathbf{G 3}$ catalyst. The volume fraction of was determined by the molar equivalents of the first monomer relative to the second monomer.

Figure S5 provides a representative example for the synthesis of poly(norbornene exo,exo-dimethylester)$b$-poly(norbornene exo,exo-di- $n$-decylester) (PN2M- $b$-PN2D). $x$ equivalents of $\mathrm{N} 2 \mathrm{M}$ relative to 1 equivalent of $\mathbf{G 3}$ were first polymerized. Upon completion (15 minutes), $y$ equivalents of N2D relative to 1 equivalent of $\mathbf{G} \mathbf{3}$ were added. The second block was allowed to react for 30 minutes. After 30 minutes, the reaction was quenched by adding excess ethyl vinyl ether and excess silica-bound metal scavenger (SiliaMetS, dimercaptotriazine [DMT]).

After stirring over SiliaMetS for 1 hour, the solution was filtered and the block polymer was precipitated into stirring cold methanol $\left(-78^{\circ} \mathrm{C}\right)$. The suspension was centrifuged at $5000 \mathrm{rpm}$ for $30 \mathrm{~s}$, then the supernatant was decanted. The polymer was dried under high vacuum to remove methanol and dichloromethane, then dissolved in benzene containing $0.1 \mathrm{wt} \%$ butylated hydroxytoluene (BHT) as a stabilizer. The solution was freeze-dried from benzene in order to afford the block polymer as either a fluffy white solid (high $f_{\mathrm{PN} 2 \mathrm{M}}$ ) or viscous light brown oil (low $\left.f_{\mathrm{PN} 2 \mathrm{M}}\right)$.

Volume fractions were verified by ${ }^{1} \mathrm{H}$ NMR as illustrated in Figures S7-S9. Unique peaks for PN2M compared to the second block (PN2B, PN1D, or PN2D) were identified by comparing (A) the corresponding monomers and (B) the PN2M homopolymer and diblock copolymer.

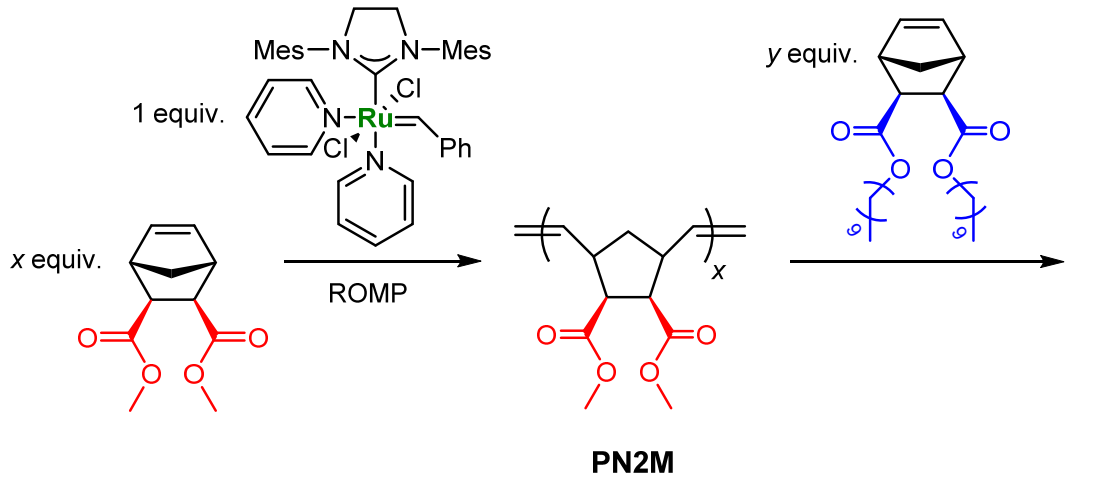

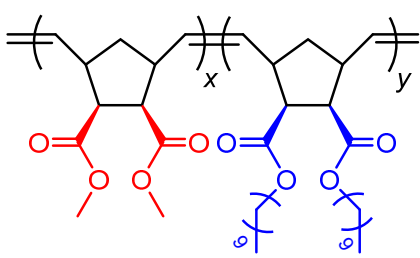

PN2M-b-PN2D

Figure S5. Representative synthesis of diblock polymers by ring-opening metathesis polymerization of norbornene exo,exo-dimethylester (N2M, red) and norbornene exo, exo-di- $n$-decylester (N2D, blue.) 
Figure S6 demonstrates how series of PN2M- $b$-PN2D block polymers were synthesized using stock solutions. Changing the volumes of added stock solutions allowed precise variations in block length and therefore composition (i.e., P $_{\mathrm{PN} 2 \mathrm{M}}$ ).

stock solutions
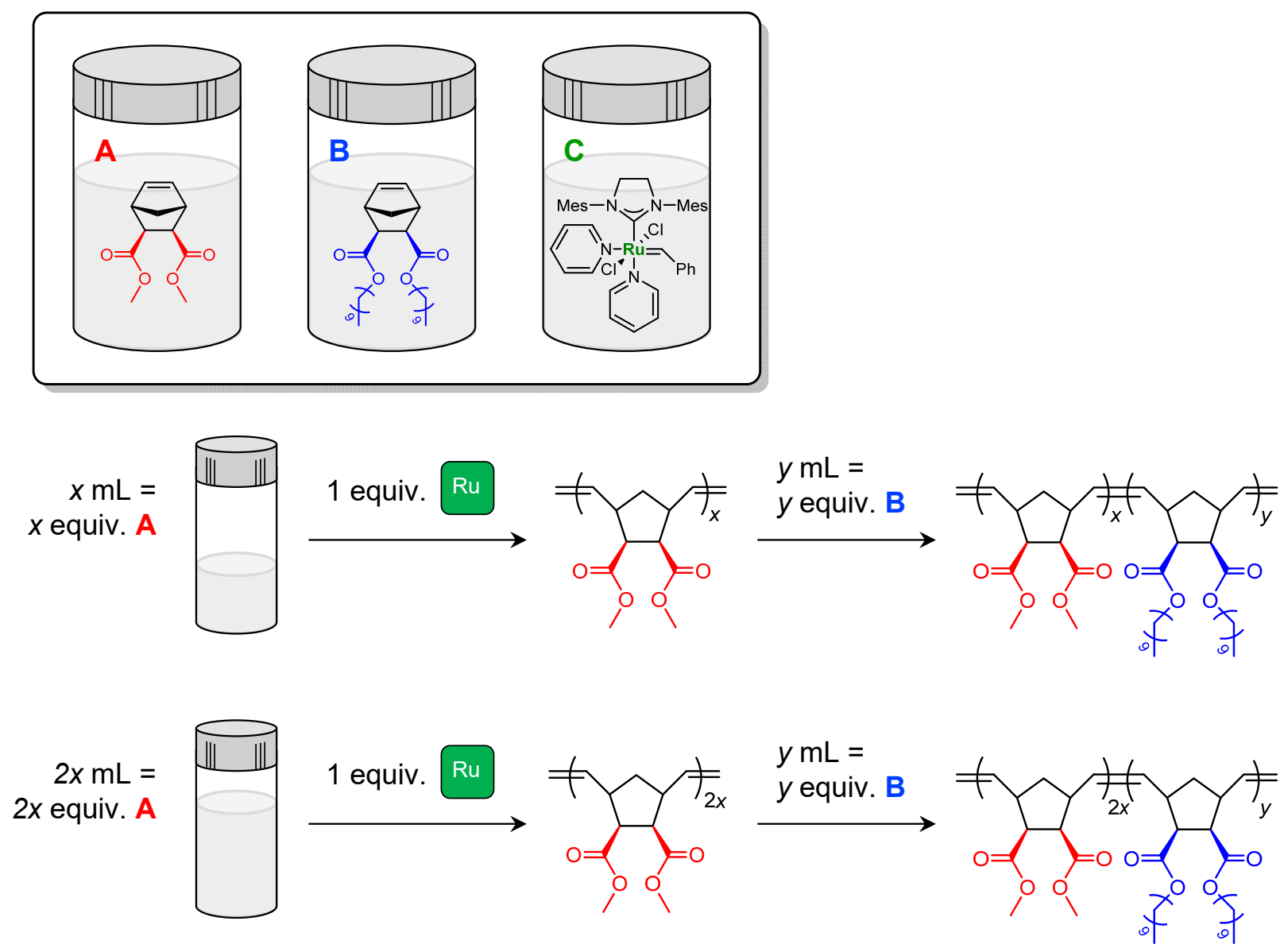

Figure S6. Schematic of synthesis of PN2M- $b$-PN2D block polymers using stock solutions. 

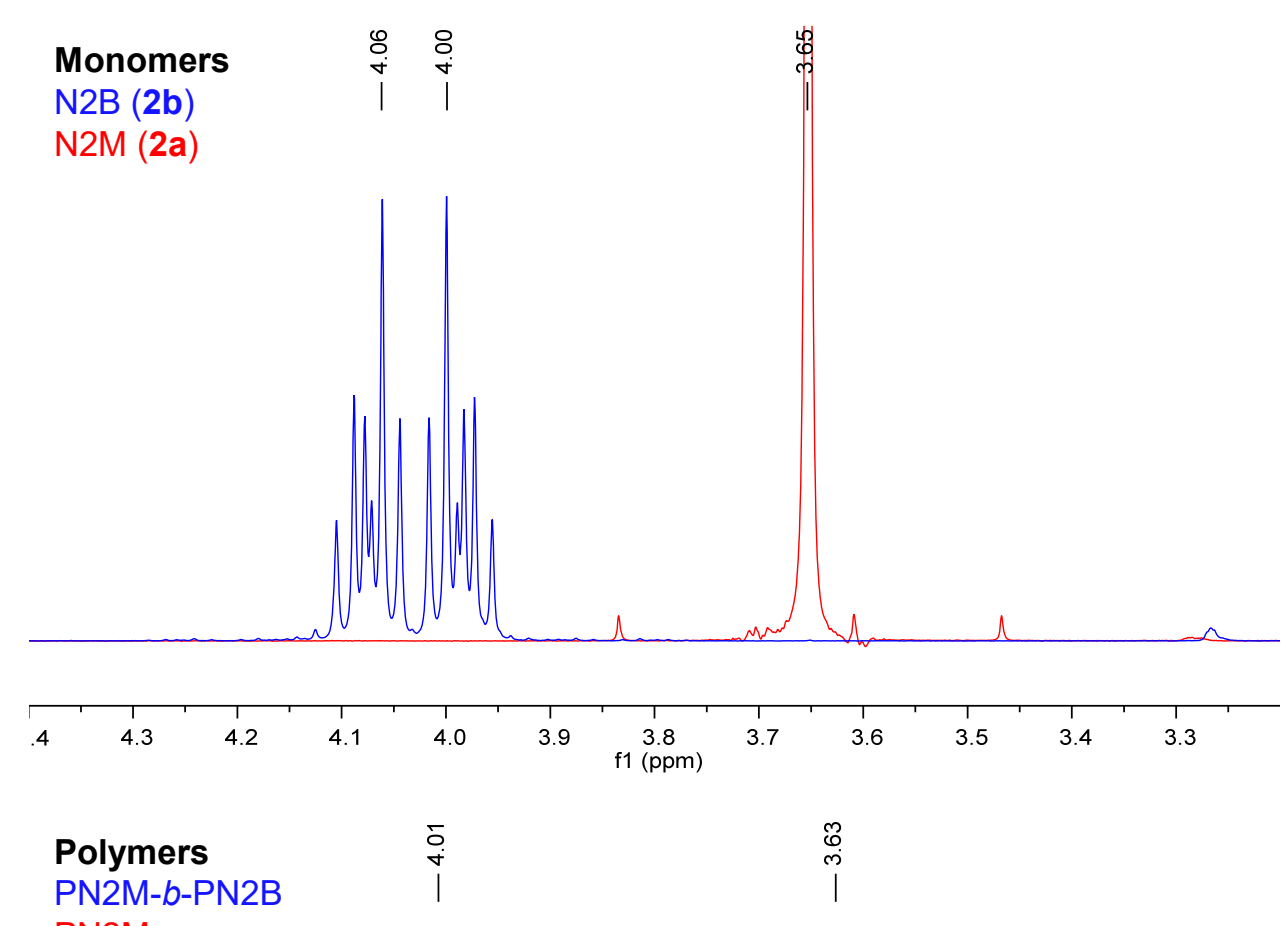

PN2M

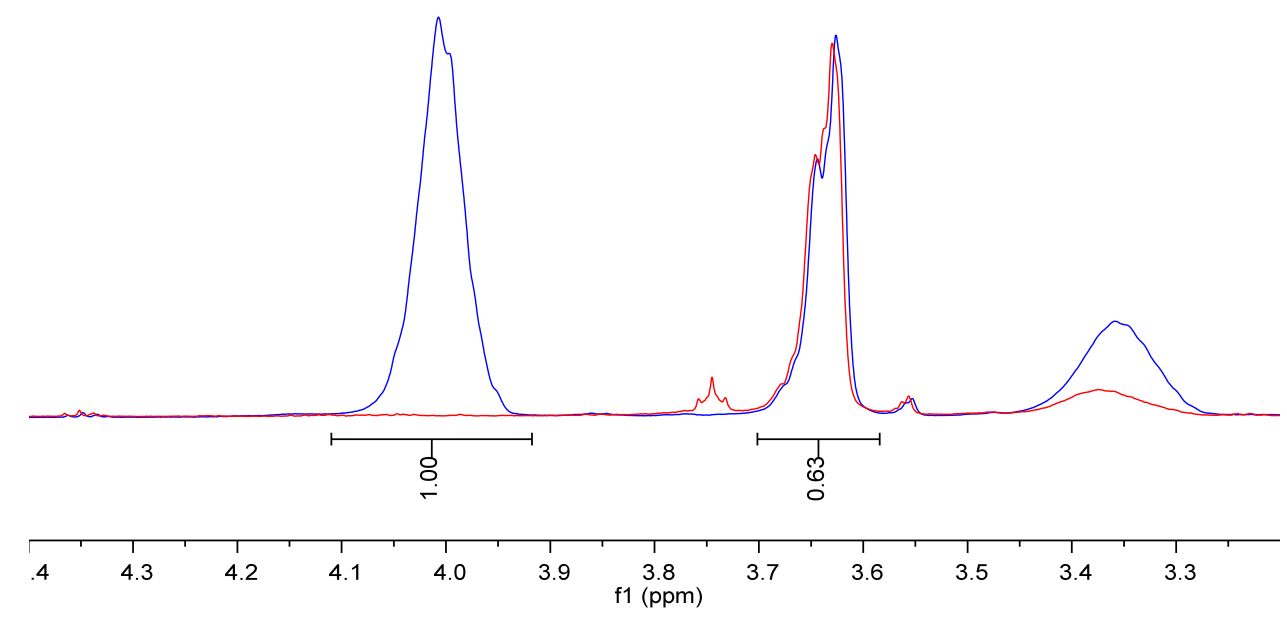

Figure S7. Superimposed ${ }^{1} \mathrm{H}$ NMR spectra (top) comparing norbornene exo,exo-dimethylester (N2M, red) and norbornene exo,exo-di- $n$-butylester $(\mathrm{N} 2 \mathrm{~B}$, blue) and (bottom) comparing the PN2M homopolymer (red) and PN2M- $b$-PN2B block polymer (blue). 

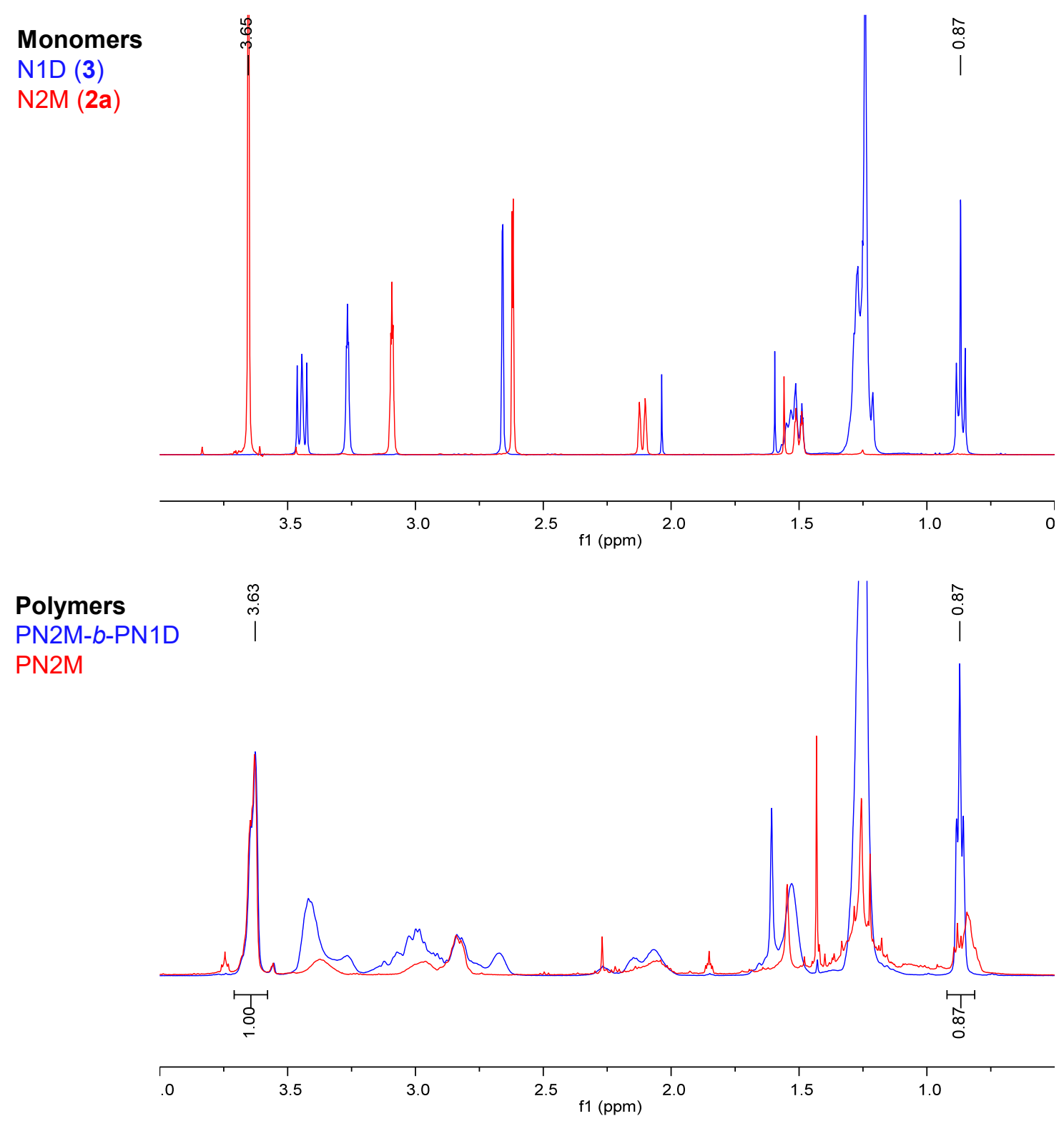

Figure S8. Superimposed ${ }^{1} \mathrm{H}$ NMR spectra (top) comparing norbornene exo, exo-dimethylester (N2M, red) and norbornene exo-n-decylimide (N1D, blue) and (bottom) comparing the PN2M homopolymer (red) and PN2M- $b$-PN1D block polymer (blue). 

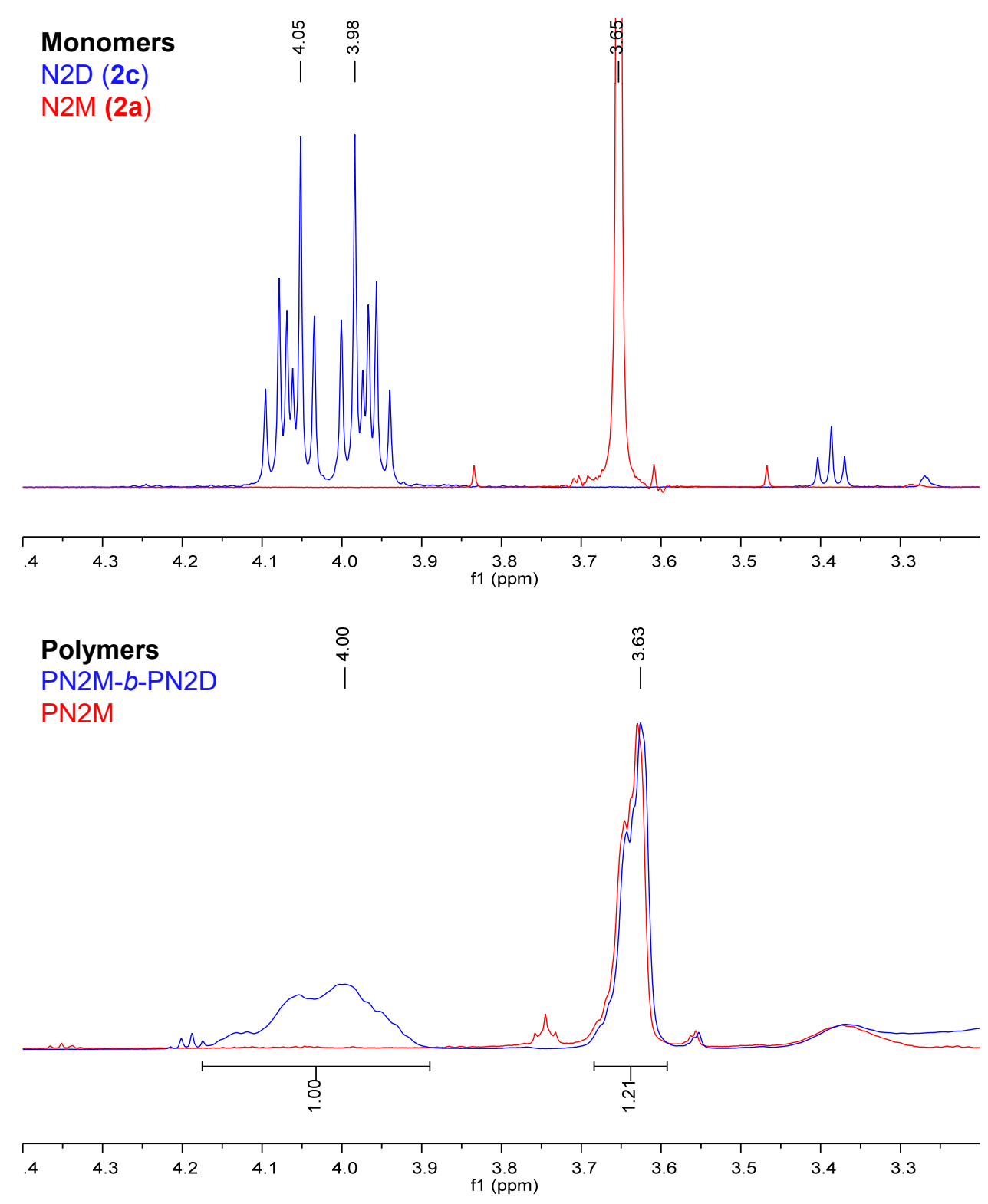

Figure S9. Superimposed ${ }^{1} \mathrm{H}$ NMR spectra (top) comparing norbornene exo,exo-dimethylester (N2M, red) and norbornene exo,exo-di- $n$-decylester (N2D, blue) and (bottom) comparing the PN2M homopolymer (red) and PN2M- $b$-PN2D block polymer (blue). 


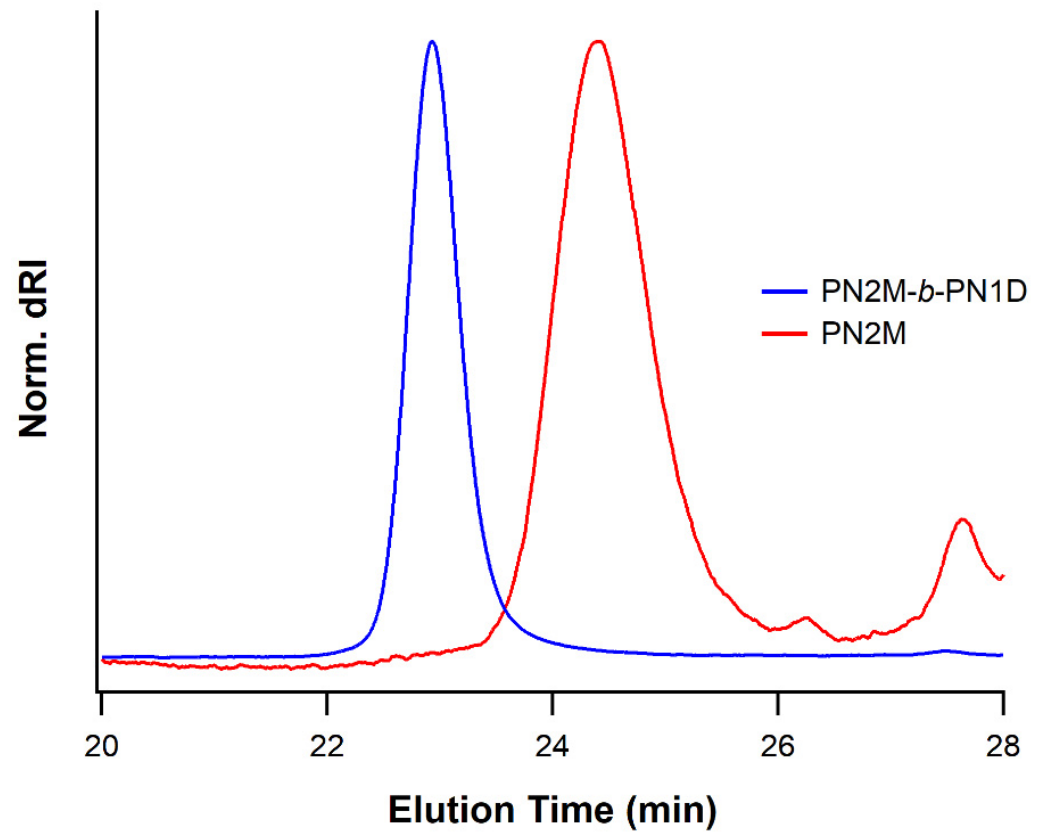

Figure S10. Representative size-exclusion chromatography (SEC) traces for block polymer B16. PN1D $(0.24,57)$, showing complete conversion of the first block (PN2M) upon addition of the second monomer, N1D.

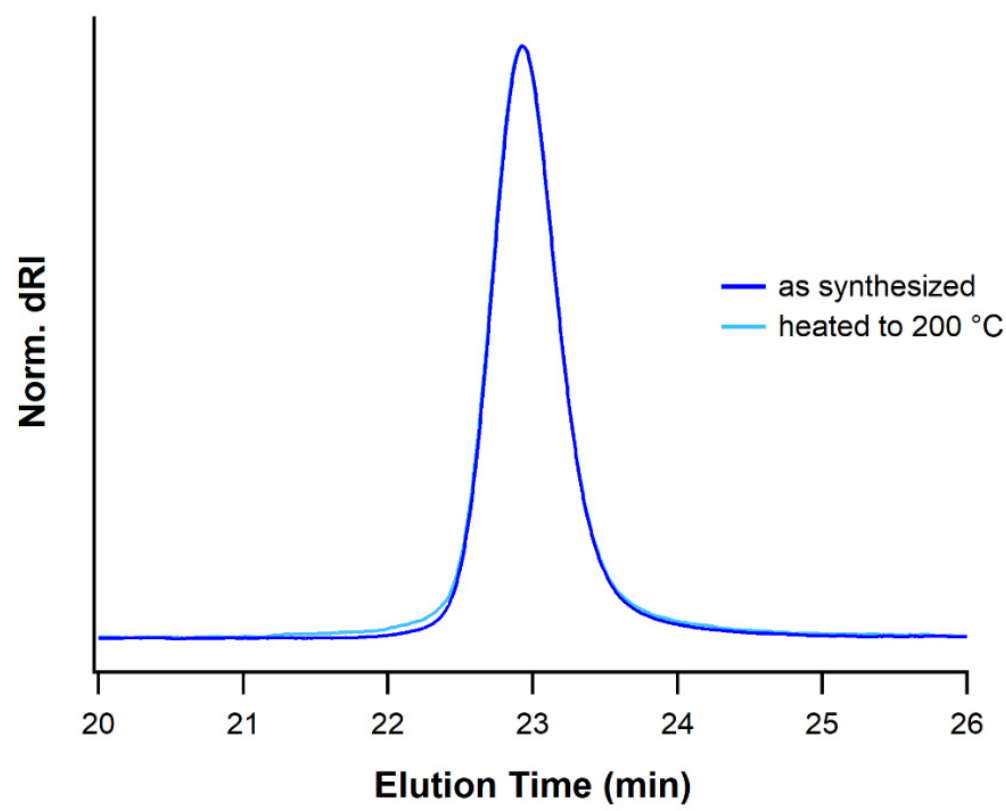

Figure S11. Comparison of size-exclusion chromatography (SEC) traces for block polymer B16. $\operatorname{PN} 1 \mathrm{D}(0.24,57)$, as-synthesized (dark blue) and after heating to $200{ }^{\circ} \mathrm{C}$ for X-ray scattering experiments (light blue). No evidence of decomposition is observed. 


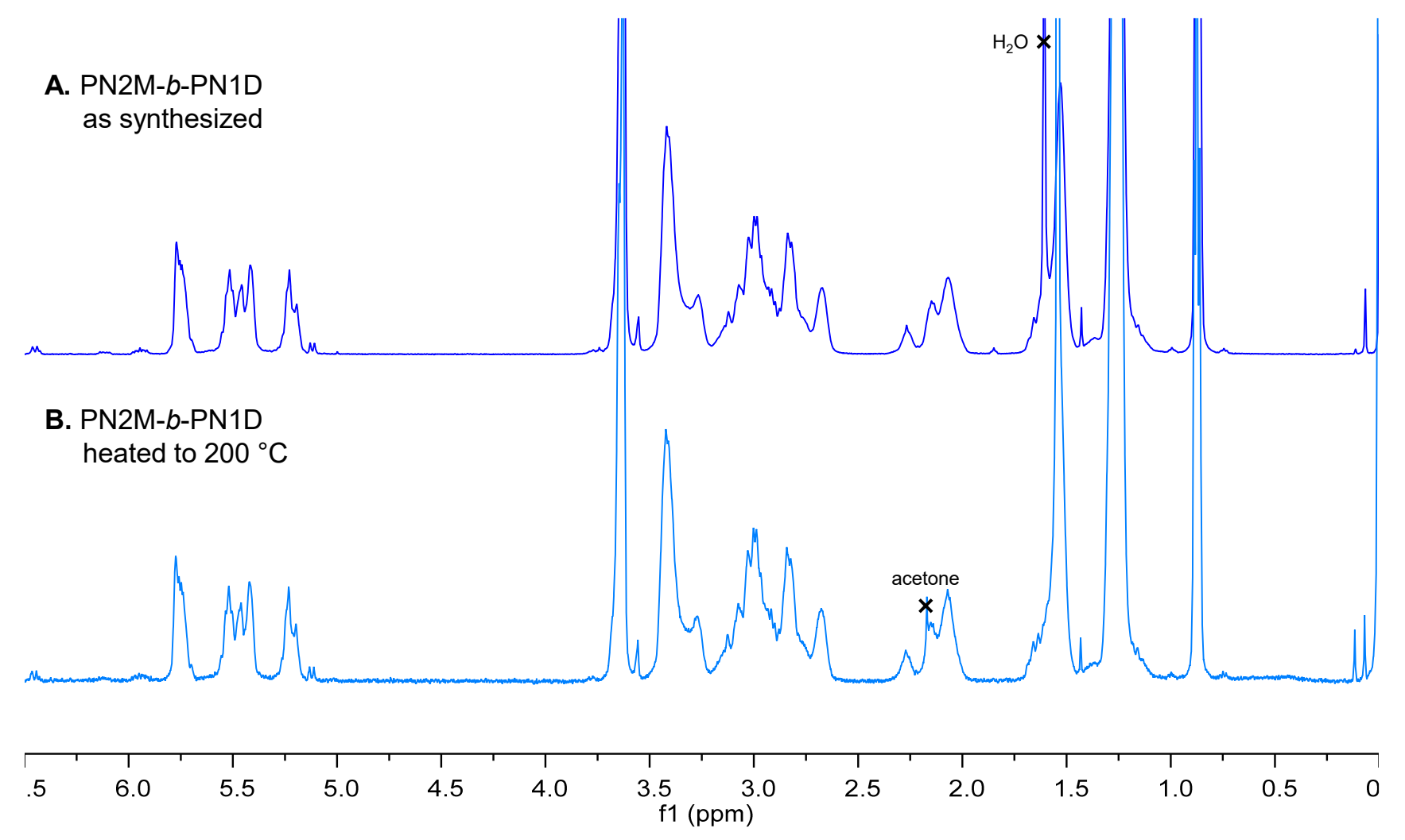

Figure S12. Comparison of ${ }^{1} \mathrm{H}$ NMR data for block polymer B16. PN1D(0.24,57) as-synthesized (top, dark blue) and after heating to $200{ }^{\circ} \mathrm{C}$ for X-ray scattering experiments (bottom, light blue). No evidence of decomposition is observed. 
Table S1. Molecular characterization data for poly(norbornene exo,exo-dimethylester)-bpoly(norbornene exo,exo-di-n-butylester) (PN2B) block polymers. Samples are labeled according to $\mathrm{PN} 2 \mathrm{~B}\left(f_{\mathrm{PN} 2 \mathrm{M}}, N_{\mathrm{bb}}\right)$, where $f_{\mathrm{PN} 2 \mathrm{M}}$ is the volume fraction of the poly(norbornene exo,exo-dimethylester) block and $N \mathrm{bb}$ is the total backbone degree of polymerization.

\begin{tabular}{|c|c|c|c|c|}
\hline ID & $f_{\mathrm{PN}_{2 \mathrm{M}}}{ }^{\mathrm{a}}$ & $N_{\mathrm{bb}}^{\mathrm{b}}$ & $M_{\mathrm{n}}(\mathrm{kDa})^{\mathrm{b}}$ & $\boldsymbol{D}^{\mathbf{b}}$ \\
\hline A1. $\mathrm{PN} 2 \mathrm{~B}(0.15,184)$ & 0.15 & 184 & 50.8 & 1.01 \\
\hline A2. $\operatorname{PN} 2 B(0.17,184)$ & 0.17 & 184 & 50.0 & 1.00 \\
\hline A3. PN2B $(0.18,159)$ & 0.18 & 159 & 43.4 & 1.04 \\
\hline A4. $\mathrm{PN} 2 \mathrm{~B}(0.18,142)$ & 0.18 & 142 & 38.5 & 1.03 \\
\hline A5. $\mathrm{PN} 2 \mathrm{~B}(0.18,129)$ & 0.18 & 129 & 35.1 & 1.04 \\
\hline A6. $\mathrm{PN} 2 \mathrm{~B}(0.21,186)$ & 0.21 & 186 & 50.0 & 1.01 \\
\hline A7. $\mathrm{PN} 2 \mathrm{~B}(0.21,156)$ & 0.21 & 156 & 41.8 & 1.04 \\
\hline A8. $\operatorname{PN} 2 B(0.21,147)$ & 0.21 & 147 & 39.6 & 1.03 \\
\hline A9. $\mathrm{PN} 2 \mathrm{~B}(0.22,124)$ & 0.22 & 124 & 33.1 & 1.04 \\
\hline A10. $\mathrm{PN} 2 \mathrm{~B}(0.22,114)$ & 0.22 & 114 & 30.5 & 1.03 \\
\hline A11. $\mathrm{PN} 2 \mathrm{~B}(0.23,184)$ & 0.23 & 184 & 48.9 & 1.01 \\
\hline A12. $\mathrm{PN} 2 \mathrm{~B}(0.23,134)$ & 0.23 & 134 & 35.7 & 1.04 \\
\hline A13. $\mathrm{PN} 2 \mathrm{~B}(0.23,121)$ & 0.23 & 121 & 32.3 & 1.03 \\
\hline A14. $\mathrm{PN} 2 \mathrm{~B}(0.23,97)$ & 0.23 & 97 & 25.9 & 1.04 \\
\hline A15. $\mathrm{PN} 2 \mathrm{~B}(0.25,131)$ & 0.25 & 131 & 34.6 & 1.02 \\
\hline A16. $\mathrm{PN} 2 \mathrm{~B}(0.26,204)$ & 0.26 & 204 & 53.8 & 1.01 \\
\hline A17. $\mathrm{PN} 2 \mathrm{~B}(0.27,133)$ & 0.27 & 133 & 34.9 & 1.02 \\
\hline A18. $\mathrm{PN} 2 \mathrm{~B}(0.28,135)$ & 0.28 & 135 & 35.2 & 1.01 \\
\hline A19. $\operatorname{PN} 2 B(0.29,183)$ & 0.29 & 183 & 47.6 & 1.01 \\
\hline A20. $\mathrm{PN} 2 \mathrm{~B}(0.18,70)$ & 0.18 & 70 & 19.0 & 1.02 \\
\hline A21. PN2B $(0.18,91)$ & 0.18 & 91 & 24.8 & 1.00 \\
\hline A22. $\mathrm{PN} 2 \mathrm{~B}(0.20,68)$ & 0.20 & 68 & 18.4 & 1.00 \\
\hline A23. $\mathrm{PN} 2 \mathrm{~B}(0.21,91)$ & 0.21 & 91 & 24.5 & 1.02 \\
\hline
\end{tabular}

${ }^{\text {a }}$ Determined from ${ }^{1} \mathrm{H}$ NMR as described in the Methods section.

${ }^{b}$ Determined from SEC as described in the Methods section using $\mathrm{d} n / \mathrm{d} c=0.0820 \mathrm{~mL} / \mathrm{g}$. 
Table S2. Molecular characterization data for poly(norbornene exo,exo-dimethylester)- $b$ poly(norbornene exo- $n$-decylester) (PN1D) block polymers. Samples are labeled according to $\mathrm{PN} 1 \mathrm{D}\left(f_{\mathrm{PN} 2 \mathrm{M}}, N_{\mathrm{bb}}\right)$, where $f_{\mathrm{PN} 2 \mathrm{M}}$ is the volume fraction of the poly(norbornene exo, exo-dimethylester) block and $N_{\mathrm{bb}}$ is the total backbone degree of polymerization.

\begin{tabular}{|c|c|c|c|c|}
\hline ID & $f_{\mathrm{PN} 2 \mathrm{M}^{\mathrm{a}}}$ & $N_{\mathbf{b b}} b^{b}$ & $M_{\mathrm{n}}(\mathrm{kDa})^{\mathrm{b}}$ & $\boldsymbol{D}^{\mathrm{b}}$ \\
\hline B1. PN1D $(0.17,97)$ & 0.17 & 97 & 26.8 & 1.01 \\
\hline B2. PN1D $(0.18,130)$ & 0.18 & 130 & 36.1 & 1.02 \\
\hline B3. PN1D $(0.21,129)$ & 0.21 & 129 & 35.1 & 1.01 \\
\hline B4. $\mathrm{PN} 1 \mathrm{D}(0.21,103)$ & 0.21 & 103 & 28.2 & 1.08 \\
\hline B5. PN1D $(0.21,89)$ & 0.21 & 89 & 24.3 & 1.01 \\
\hline B6. $\mathrm{PN} 1 \mathrm{D}(0.21,78)$ & 0.21 & 78 & 21.1 & 1.00 \\
\hline B7. PN1D $(0.22,101)$ & 0.22 & 101 & 27.4 & 1.01 \\
\hline B8. PN1D $(0.22,91)$ & 0.22 & 91 & 24.6 & 1.01 \\
\hline B9. $\mathrm{PN} 1 \mathrm{D}(0.22,77)$ & 0.22 & 77 & 20.9 & 1.01 \\
\hline B10. $\operatorname{PN} 1 \mathrm{D}(0.23,126)$ & 0.23 & 126 & 34.0 & 1.02 \\
\hline B11. PN1D $(0.25,129)$ & 0.25 & 129 & 34.5 & 1.03 \\
\hline B12. $\mathrm{PN} 1 \mathrm{D}(0.26,95)$ & 0.26 & 95 & 25.2 & 1.10 \\
\hline B13. PN1D $(0.26,90)$ & 0.26 & 90 & 24.0 & 1.01 \\
\hline B14. PN1D $(0.26,78)$ & 0.26 & 78 & 20.9 & 1.04 \\
\hline B15. PN1D $(0.24,44)$ & 0.24 & 44 & 11.9 & 1.01 \\
\hline B16. PN1D $(0.24,57)$ & 0.24 & 57 & 15.5 & 1.07 \\
\hline B17. PN1D $(0.27,47)$ & 0.27 & 47 & 12.5 & 1.04 \\
\hline B18. PN1D $(0.29,57)$ & 0.29 & 57 & 15.0 & 1.00 \\
\hline
\end{tabular}

${ }^{\text {a }}$ Determined from ${ }^{1} \mathrm{H}$ NMR as described in the Methods section.

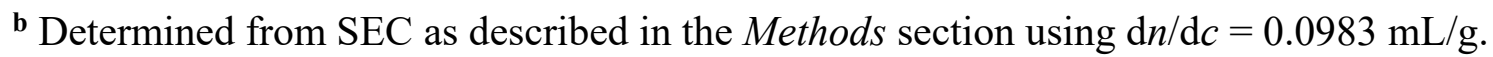


Table S3. Molecular characterization data for poly(norbornene exo,exo-dimethylester)-bpoly(norbornene exo,exo-di-n-decylester) (PN2D) block polymers. Samples are labeled according to $\mathrm{PN} 2 \mathrm{D}\left(f_{\mathrm{PN} 2 \mathrm{M}}, N_{\mathrm{bb}}\right)$, where $f_{\mathrm{PN} 2 \mathrm{M}}$ is the volume fraction of the poly(norbornene exo,exo-dimethylester) block and $N_{\mathrm{bb}}$ is the total backbone degree of polymerization.

\begin{tabular}{|c|c|c|c|c|}
\hline ID & $f_{\mathrm{PN} 2 \mathrm{M}^{\mathrm{a}}}$ & $N_{\mathrm{bb}} \mathrm{b}^{\mathrm{b}}$ & $M_{\mathrm{n}}(\mathbf{k D a})^{\mathrm{b}}$ & $\boldsymbol{D}^{\mathbf{b}}$ \\
\hline C1. PN2D $(0.15,114)$ & 0.15 & 114 & 42.7 & 1.01 \\
\hline C2. PN2D $(0.15,102)$ & 0.15 & 102 & 38.3 & 1.01 \\
\hline C3. $\mathrm{PN} 2 \mathrm{D}(0.17,86)$ & 0.17 & 86 & 31.9 & 1.01 \\
\hline C4. PN2D $(0.19,76)$ & 0.19 & 76 & 27.3 & 1.00 \\
\hline C5. $\mathrm{PN} 2 \mathrm{D}(0.20,86)$ & 0.20 & 86 & 30.6 & 1.01 \\
\hline C6. $\mathrm{PN} 2 \mathrm{D}(0.20,78)$ & 0.20 & 78 & 27.6 & 1.01 \\
\hline C7. PN2D $(0.21,64)$ & 0.21 & 64 & 22.5 & 1.02 \\
\hline C8. $\operatorname{PN} 2 \mathrm{D}(0.17,78)$ & 0.17 & 78 & 28.9 & 1.05 \\
\hline C9. $\mathrm{PN} 2 \mathrm{D}(0.17,53)$ & 0.17 & 53 & 19.5 & 1.00 \\
\hline C10. PN2D $(0.20,54)$ & 0.20 & 54 & 19.3 & 1.01 \\
\hline C11. PN2D $(0.22,52)$ & 0.22 & 52 & 18.1 & 1.01 \\
\hline C12. PN2D $(0.24,47)$ & 0.24 & 47 & 16.2 & 1.01 \\
\hline C13. PN2D $(0.25,57)$ & 0.25 & 57 & 19.4 & 1.01 \\
\hline C14. PN2D $(0.26,46)$ & 0.26 & 46 & 15.4 & 1.02 \\
\hline C15. PN2D $(0.27,41)$ & 0.27 & 41 & 13.7 & 1.02 \\
\hline C16. PN2D $(0.30,43)$ & 0.30 & 43 & 13.8 & 1.01 \\
\hline C17. PN2D $(0.29,58)$ & 0.29 & 58 & 18.8 & 1.10 \\
\hline C18. PN2D $(0.29,48)$ & 0.29 & 48 & 15.7 & 1.01 \\
\hline
\end{tabular}

${ }^{\text {a }}$ Determined from ${ }^{1} \mathrm{H}$ NMR as described in the Methods section.

${ }^{b}$ Determined from SEC as described in the Methods section using $\mathrm{d} n / \mathrm{d} c=0.0790 \mathrm{~mL} / \mathrm{g}$. 


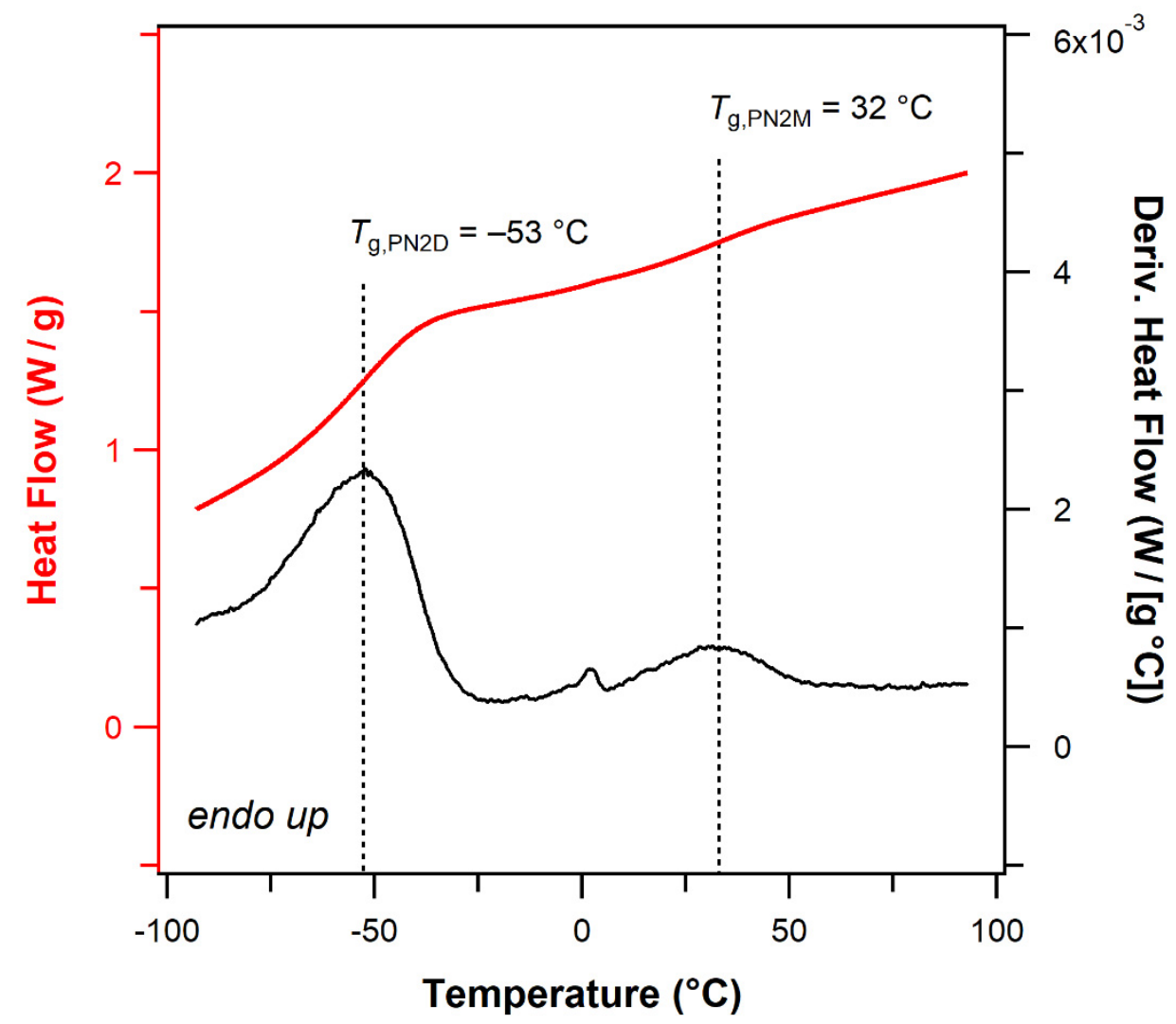

Figure S13. Differential scanning calorimetry (DSC) data for C11. PN2D(0.22,52). Data were collected on the second heating ramp, from $-100{ }^{\circ} \mathrm{C}$ to $100{ }^{\circ} \mathrm{C}$ at $10{ }^{\circ} \mathrm{C} / \mathrm{min}$. The glass transition temperatures $\left(T_{\mathrm{g}}\right)$ for the PN2M and PN2D blocks are indicated. 
Table S4. Morphologies assigned for each poly(exo,exo-norbornene dimethylester)-b-poly(norbornene exo,exo-di- $n$-butylester) [PN2B( $\left.\left.f_{\mathrm{PN} 2 \mathrm{M}}, N_{\mathrm{bb}}\right)\right]$ block polymer. Samples were annealed at $140{ }^{\circ} \mathrm{C}$ for $24 \mathrm{~h}$ as described in the Methods section. Synchrotron-source SAXS data were collected on heating between 25 and $200{ }^{\circ} \mathrm{C}$. Samples were heated to each temperature at $10{ }^{\circ} \mathrm{C} / \mathrm{min}$ and annealed for 15 minutes prior to collecting data. The 1D-averaged SAXS data at each temperature is provided in Figure S14.

\begin{tabular}{|c|c|c|c|c|c|}
\hline ID & $25^{\circ} \mathrm{C}$ & $100{ }^{\circ} \mathrm{C}$ & 140 or $150^{\circ} \mathrm{C}$ & 170 or $175^{\circ} \mathrm{C}$ & $200^{\circ} \mathrm{C}$ \\
\hline A1. PN2B $(0.15,184)$ & $\mathrm{BCC}$ & $\mathrm{BCC}$ & $\mathrm{BCC}$ & $\mathrm{BCC}$ & $\mathrm{BCC}$ \\
\hline A2. $\mathrm{PN} 2 \mathrm{~B}(0.17,184)$ & $\mathrm{BCC}$ & $\mathrm{BCC}$ & $\mathrm{BCC}$ & $\mathrm{BCC}$ & $\mathrm{BCC}$ \\
\hline A3. $\mathrm{PN} 2 \mathrm{~B}(0.18,159)$ & $\mathrm{BCC}$ & $\mathrm{BCC}$ & $\mathrm{BCC}$ & $\mathrm{BCC}$ & DIS \\
\hline A4. PN2B $(0.18,142)$ & $\mathrm{BCC}$ & $\mathrm{BCC}$ & $\mathrm{BCC}$ & DIS & DIS \\
\hline A5. PN2B $(0.18,129)$ & $\mathrm{BCC}$ & $\mathrm{BCC}$ & DIS & DIS & DIS \\
\hline A6. PN2B $(0.21,186)$ & $\mathrm{HEX}_{\mathrm{C}}$ & $\mathrm{HEX}_{\mathrm{C}}$ & $\mathrm{HEX}_{\mathrm{C}}$ & $\mathrm{HEX}_{\mathrm{C}}$ & $\mathrm{HEX}_{\mathrm{C}}$ \\
\hline A7. PN2B $(0.21,156)$ & $\mathrm{HEX}_{\mathrm{C}}$ & $\mathrm{HEX}_{\mathrm{C}}$ & $\mathrm{HEX}_{\mathrm{C}}$ & $\mathrm{HEX}_{\mathrm{C}}$ & $\mathrm{HEX}_{\mathrm{C}}$ \\
\hline A8. PN2B $(0.21,147)$ & HEX & $\mathrm{HEX}_{\mathrm{c}}$ & $\mathrm{HEX}_{\mathrm{C}}$ & $\mathrm{HEX}_{\mathrm{C}}$ & DIS \\
\hline A9. PN2B $(0.22,124)$ & HEX & $\mathrm{HEX}_{\mathrm{c}}$ & $\mathrm{HEX}_{\mathrm{c}}$ & DIS & DIS \\
\hline A10. PN2B(0.22,114) & $\mathrm{HEX}_{\mathrm{C}}$ & $\mathrm{HEX}_{\mathrm{C}}$ & DIS & DIS & DIS \\
\hline A11. PN2B(0.23,184) & $\mathrm{HEX}_{\mathrm{c}}$ & $\mathrm{HEX}_{\mathrm{c}}$ & $\mathrm{HEX}_{\mathrm{c}}$ & $\mathrm{HEX}_{\mathrm{c}}$ & $\mathrm{HEX}_{\mathrm{c}}$ \\
\hline A12. PN2B $(0.23,134)$ & $\mathrm{HEX}_{\mathrm{C}}$ & $\mathrm{HEX}_{\mathrm{C}}$ & $\mathrm{HEX}_{\mathrm{C}}$ & $\mathrm{HEX}_{\mathrm{C}}$ & DIS \\
\hline A13. PN2B $(0.23,121)$ & $\mathrm{HEX}_{\mathrm{C}}$ & $\mathrm{HEX}_{\mathrm{C}}$ & $\mathrm{HEX}_{\mathrm{C}}$ & DIS & DIS \\
\hline A14. $\mathrm{PN} 2 \mathrm{~B}(0.23,97)$ & $\mathrm{HEX}_{\mathrm{c}}$ & HEXc & DIS & DIS & DIS \\
\hline A15. PN2B $(0.25,131)$ & $\mathrm{HEX}_{\mathrm{c}}$ & HEXc & $\mathrm{HEX}_{\mathrm{c}}$ & $\mathrm{HEX}_{\mathrm{c}}$ & $\mathrm{HEX}_{\mathrm{c}}$ \\
\hline A16. PN2B(0.26,204) & $\mathrm{HEX}_{\mathrm{c}}$ & HEXc & $\mathrm{HEX}_{\mathrm{c}}$ & $\mathrm{HEX}_{\mathrm{C}}$ & $\mathrm{HEX}_{\mathrm{c}}$ \\
\hline A17. PN2B(0.27,133) & $\mathrm{HEX}_{\mathrm{c}}$ & $\mathrm{HEX}_{\mathrm{C}}$ & $\mathrm{HEX}_{\mathrm{C}}$ & $\mathrm{HEX}_{\mathrm{c}}$ & $\mathrm{HEX}_{\mathrm{c}}$ \\
\hline A18. PN2B $(0.28,135)$ & $\mathrm{HEX}_{\mathrm{c}}$ & $\mathrm{HEX}_{\mathrm{C}}$ & $\mathrm{HEX}_{\mathrm{c}}$ & $\mathrm{HEX}_{\mathrm{C}}$ & $\mathrm{HEX}_{\mathrm{c}}$ \\
\hline A19. PN2B(0.29,183) & $\mathrm{HEX}_{\mathrm{c}}$ & $\mathrm{HEX}_{\mathrm{C}}$ & $\mathrm{HEX}_{\mathrm{c}}$ & $\mathrm{HEX}_{\mathrm{c}}$ & $\mathrm{HEX}_{\mathrm{c}}$ \\
\hline A20. $\operatorname{PN} 2 B(0.18,70)$ & DIS & DIS & DIS & DIS & DIS \\
\hline A21. $\operatorname{PN} 2 B(0.18,91)$ & DIS & DIS & DIS & DIS & DIS \\
\hline A22. $P N 2 B(0.20,68)$ & DIS & DIS & DIS & DIS & DIS \\
\hline A23. $P N 2 B(0.21,91)$ & DIS & DIS & DIS & DIS & DIS \\
\hline
\end{tabular}


Table S5. Morphologies assigned for each poly(exo,exo-norbornene dimethylester)- $b$-poly(norbornene exo- $n$-decylimide) $\left[\mathrm{PN} 1 \mathrm{D}\left(f_{\mathrm{PN} 2 \mathrm{M}}, N_{\mathrm{bb}}\right)\right]$ block polymer. Samples were annealed at $140{ }^{\circ} \mathrm{C}$ for $24 \mathrm{~h}$ as described in the Methods section. Synchrotron-source SAXS data were collected on heating between 25 and $200{ }^{\circ} \mathrm{C}$. Samples were heated to each temperature at $10{ }^{\circ} \mathrm{C} / \mathrm{min}$ and annealed for 15 minutes prior to collecting data. The 1D-averaged SAXS data is provided in Figure S15.

\begin{tabular}{|c|c|c|c|c|c|}
\hline ID & $25^{\circ} \mathrm{C}$ & $100^{\circ} \mathrm{C}$ & $140^{\circ} \mathrm{C}$ & $170^{\circ} \mathrm{C}$ & $200^{\circ} \mathrm{C}$ \\
\hline B1. PN1D $(0.17,97)$ & $\mathrm{BCC}$ & $\mathrm{BCC}$ & $\mathrm{BCC}$ & DIS & DIS \\
\hline B2. $\operatorname{PN} 1 \mathrm{D}(0.18,130)$ & HEXc & $\mathrm{HEX}_{\mathrm{c}}$ & HEXc & $\mathrm{HEX}_{\mathrm{C}}+\mathrm{BCC}$ & $\mathrm{BCC}$ \\
\hline B3. PN1D $(0.21,129)$ & HEXc & $\mathrm{HEX}_{\mathrm{c}}$ & $\mathrm{HEX}_{\mathrm{c}}$ & $\mathrm{HEX}_{\mathrm{C}}$ & $\mathrm{HEX}_{\mathrm{c}}$ \\
\hline B4. $P N 1 D(0.21,103)$ & HEXc & $\mathrm{HEX}_{\mathrm{c}}$ & HEXc & $\mathrm{HEX}_{\mathrm{c}}$ & DIS \\
\hline B5. PN1D $(0.21,89)$ & HEXc & HEXc $_{c}$ & HEXc & DIS & DIS \\
\hline B6. PN1D $(0.21,78)$ & $\mathrm{HEX}_{\mathrm{C}}$ & $\mathrm{HEX}_{\mathrm{c}}$ & DIS & DIS & DIS \\
\hline B7. PN1D $(0.22,101)$ & $\mathrm{HEX}_{\mathrm{C}}$ & $\mathrm{HEX}_{\mathrm{C}}$ & $\mathrm{HEX}_{\mathrm{C}}$ & $\mathrm{HEX}_{\mathrm{C}}$ & $\mathrm{HEX}_{\mathrm{C}}$ \\
\hline B8. $\operatorname{PN1D}(0.22,91)$ & $\mathrm{HEX}_{\mathrm{C}}$ & $\mathrm{HEX}_{\mathrm{C}}$ & $\mathrm{HEX}_{\mathrm{C}}$ & $\mathrm{HEX}_{\mathrm{C}}$ & DIS \\
\hline B9. PN1D $(0.22,77)$ & $\mathrm{HEX}_{\mathrm{C}}$ & $\mathrm{HEX}_{\mathrm{C}}$ & DIS & DIS & DIS \\
\hline B10. PN1D $(0.23,126)$ & $\mathrm{HEX}_{\mathrm{C}}$ & $\mathrm{HEX}_{\mathrm{C}}$ & $\mathrm{HEX}_{\mathrm{C}}$ & $\mathrm{HEX}_{\mathrm{C}}$ & $\mathrm{HEX}_{\mathrm{C}}$ \\
\hline B11. PN1D $(0.25,129)$ & $\mathrm{HEX}_{\mathrm{C}}$ & $\mathrm{HEX}_{\mathrm{c}}$ & $\mathrm{HEX}_{\mathrm{C}}$ & $\mathrm{HEX}_{\mathrm{C}}$ & $\mathrm{HEX}_{\mathrm{C}}$ \\
\hline B12. $P N 1 D(0.26,95)$ & $\mathrm{HEX}_{\mathrm{C}}$ & $\mathrm{HEX}_{\mathrm{C}}$ & $\mathrm{HEX}_{\mathrm{C}}$ & $\mathrm{HEX}_{C}+\mathrm{DIS}$ & $\mathrm{HEX}_{\mathrm{C}}+\mathrm{DIS}$ \\
\hline B13. $P N 1 D(0.26,90)$ & $\mathrm{HEX}_{\mathrm{c}}$ & $\mathrm{HEX}_{\mathrm{c}}$ & HEXc & HEXc & $\mathrm{HEX}_{\mathrm{C}}$ \\
\hline B14. $P N 1 D(0.26,78)$ & $\mathrm{HEX}_{\mathrm{C}}$ & HEXc & HEXc & DIS & DIS \\
\hline B15. PN1D $(0.24,44)$ & DIS & DIS & DIS & DIS & DIS \\
\hline B16. PN1D $(0.24,57)$ & DIS & DIS & DIS & DIS & DIS \\
\hline B17. PN1D $(0.27,47)$ & DIS & DIS & DIS & DIS & DIS \\
\hline B18. PN1D $(0.29,57)$ & DIS & DIS & DIS & DIS & DIS \\
\hline
\end{tabular}


Table S6. Morphologies assigned for each poly(exo,exo-norbornene dimethylester)- $b$-poly(norbornene exo,exo-di- $n$-decylester) $\left[\mathrm{PN} 2 \mathrm{D}\left(f_{\mathrm{PN} 2 \mathrm{M}}, N_{\mathrm{bb}}\right)\right]$ block polymer. Samples were annealed at $140{ }^{\circ} \mathrm{C}$ for $24 \mathrm{~h}$ as described in the Methods section. Synchrotron-source SAXS data were collected on heating between 25 and $200{ }^{\circ} \mathrm{C}$. Samples were heated to each temperature at $10{ }^{\circ} \mathrm{C} / \mathrm{min}$ and annealed for 15 minutes prior to collecting data. The 1D-averaged SAXS data is provided in Figure S16.

\begin{tabular}{|c|c|c|c|c|c|}
\hline ID & $25^{\circ} \mathrm{C}$ & $100^{\circ} \mathrm{C}$ & 140 or $150^{\circ} \mathrm{C}$ & 170 or $175^{\circ} \mathrm{C}$ & $200^{\circ} \mathrm{C}$ \\
\hline C1. PN2D $(0.15,114)$ & LLP & LLP & LLP & LLP & LLP \\
\hline C2. PN2D $(0.15,102)$ & LLP & LLP & LLP & LLP & LLP \\
\hline C3. PN2D $(0.17,86)$ & LLP & LLP & LLP & LLP & LLP \\
\hline C4. PN2D $(0.19,76)$ & LLP & LLP & LLP & LLP & LLP \\
\hline C5. PN2D $(0.20,86)$ & LLP & LLP & LLP & LLP & LLP \\
\hline C6. PN2D $(0.20,78)$ & LLP & LLP & LLP & LLP & LLP \\
\hline C7. PN2D $(0.21,64)$ & LLP & LLP & LLP & $\mathrm{LLP}+\mathrm{BCC}$ & $\mathrm{BCC}$ \\
\hline C8. PN2D $(0.17,78)$ & $\mathrm{BCC}$ & $\mathrm{BCC}$ & $\mathrm{BCC}$ & $\mathrm{BCC}$ & $\mathrm{BCC}$ \\
\hline C9. PN2D $(0.17,53)$ & $\mathrm{BCC}$ & $\mathrm{BCC}$ & $\mathrm{BCC}$ & DIS & DIS \\
\hline C10. PN2D $(0.20,54)$ & DDQC & DDQC & DDQC & DDQC & $\mathrm{BCC}$ \\
\hline C11. PN2D $(0.22,52)$ & DDQC & DDQC & DDQC & DDQC & sigma \\
\hline C12. $\mathrm{PN} 2 \mathrm{D}(0.24,47)$ & DDQC & DDQC & DDQC & DDQC & DIS \\
\hline C13. PN2D $(0.25,57)$ & DDQC & DDQC & DDQC & DDQC & sigma \\
\hline C14. PN2D $(0.26,46)$ & DDQC & DDQC & DDQC & DDQC & A15 \\
\hline C15. PN2D $(0.27,41)$ & $\mathrm{HEX}_{\mathrm{C}}$ & $\operatorname{HEX}_{\mathrm{C}}$ & $\operatorname{HEX}_{\mathrm{C}}$ & DIS & DIS \\
\hline C16. PN2D $(0.30,43)$ & $\operatorname{HEX}_{\mathrm{C}}$ & $\operatorname{HEX}_{\mathrm{C}}$ & $\mathrm{HEX}_{\mathrm{C}}$ & $\operatorname{HEX}_{\mathrm{C}}$ & DIS \\
\hline C17. PN2D $(0.29,58)$ & $\mathrm{A} 15+\mathrm{HEX}_{\mathrm{C}}$ & $\mathrm{A} 15+\mathrm{HEX}_{\mathrm{C}}$ & $\mathrm{A} 15+\mathrm{HEX}_{\mathrm{C}}$ & $\operatorname{HEX}_{\mathrm{C}}$ & $\operatorname{HEX}_{\mathrm{C}}$ \\
\hline C18. PN2D $(0.29,48)$ & $\mathrm{A} 15+\mathrm{HEX}_{\mathrm{C}}$ & $\mathrm{A} 15+\mathrm{HEX}_{\mathrm{C}}$ & $\mathrm{A} 15+\mathrm{HEX}_{\mathrm{C}}$ & $\mathrm{HEX}_{\mathrm{C}}$ & $\mathrm{HEX}_{\mathrm{C}}$ \\
\hline
\end{tabular}




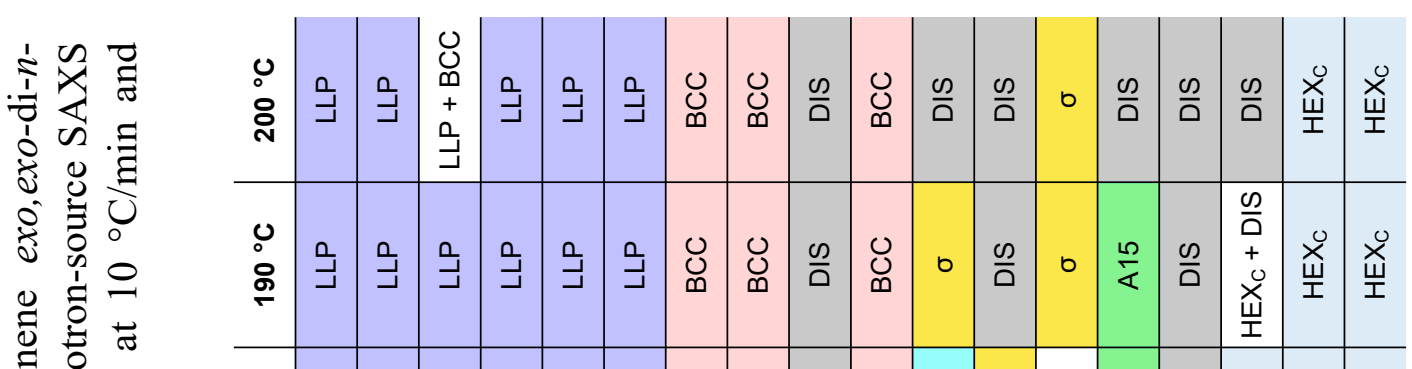

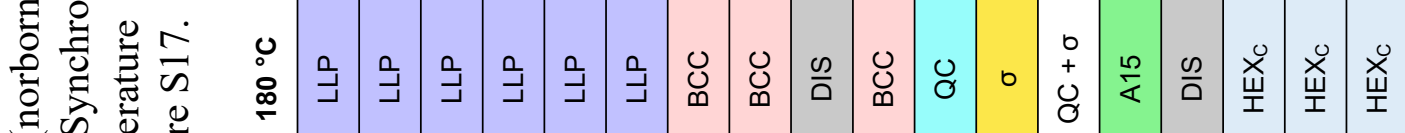

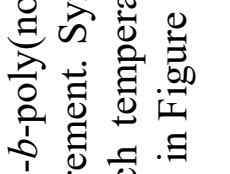

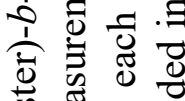

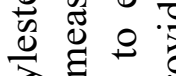

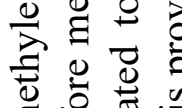

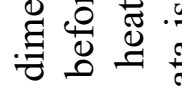

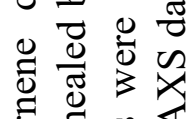

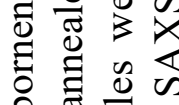

要

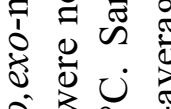

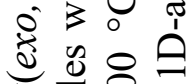

농 웡

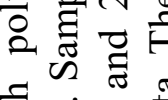

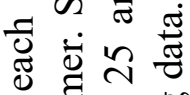

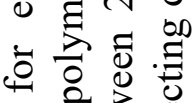

उ

.5.5.

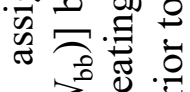

क ₹

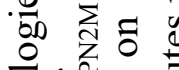

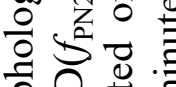

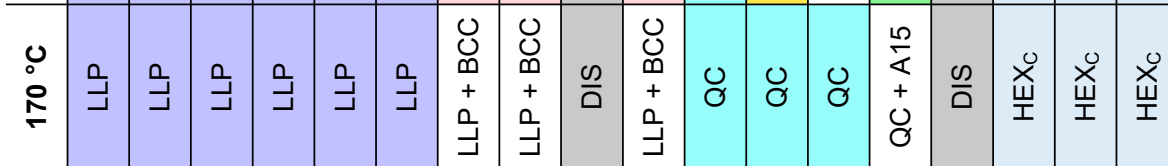

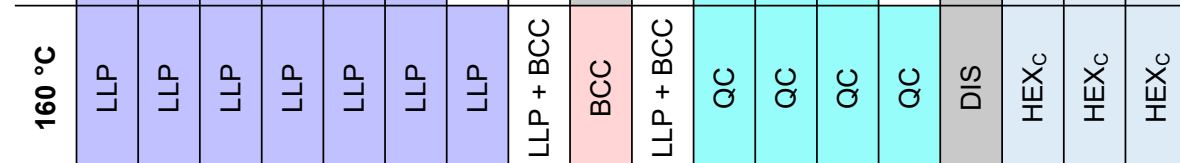

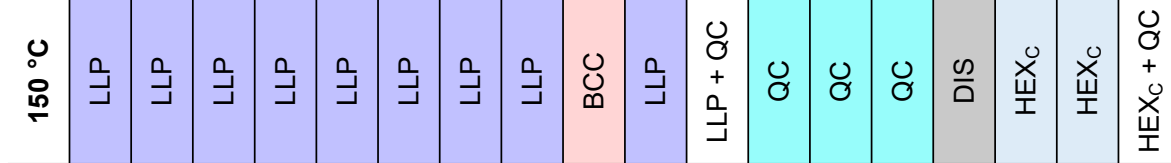

\begin{tabular}{l}
\hline 0 \\
0
\end{tabular}

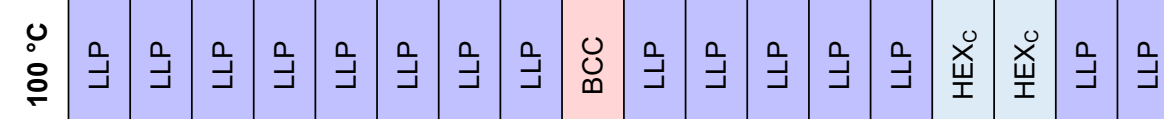

룽 ते

$\sum^{2}=$

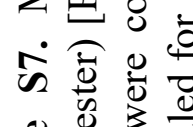

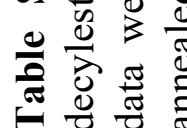

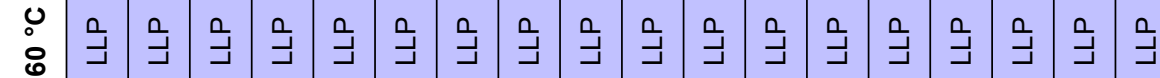

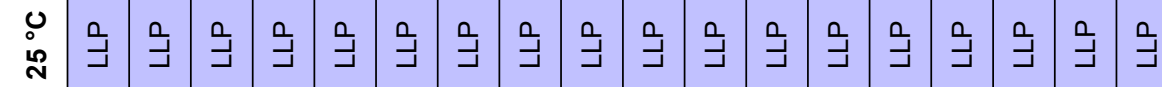

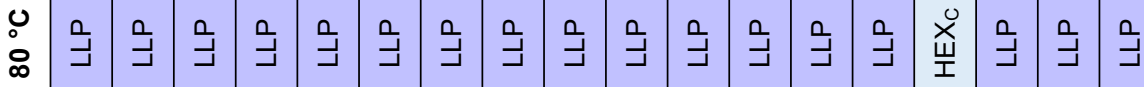

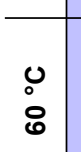

모 모 몸

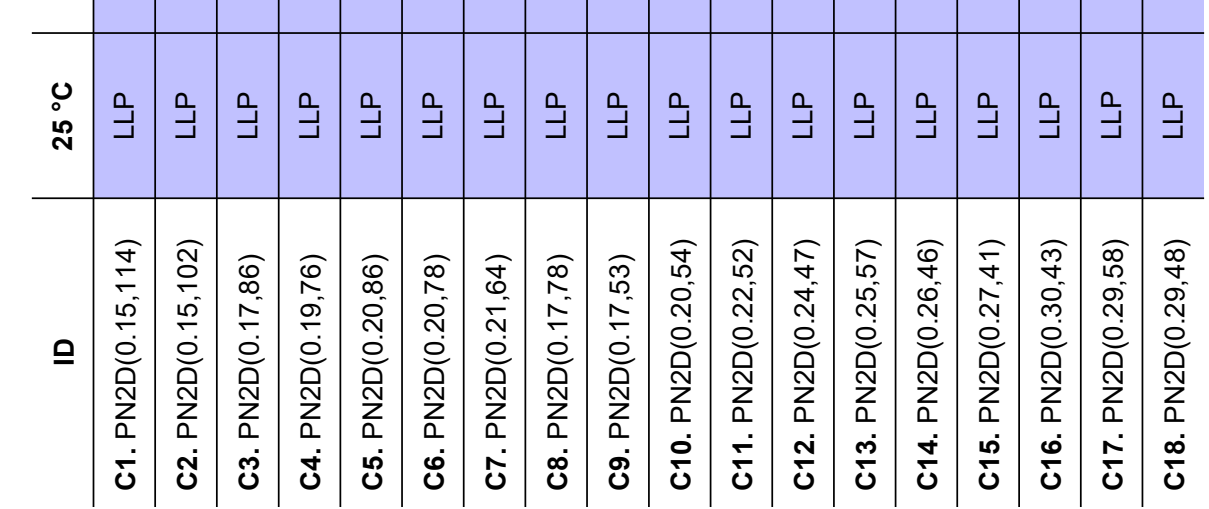


Figure S14 (Page 1/4). 1D azimuthally averaged SAXS patterns for poly(exo,exo-norbornene dimethylester)- $b$-poly(norbornene exo,exo-di- $n$-butylester) (PN2B) block polymers. Samples were annealed as described in the Methods section. Synchrotron-source SAXS data were collected on heating between 25 and $200{ }^{\circ} \mathrm{C}$. Samples were heated to each temperature at $10{ }^{\circ} \mathrm{C} / \mathrm{min}$ and annealed for 15 minutes prior to collecting data.
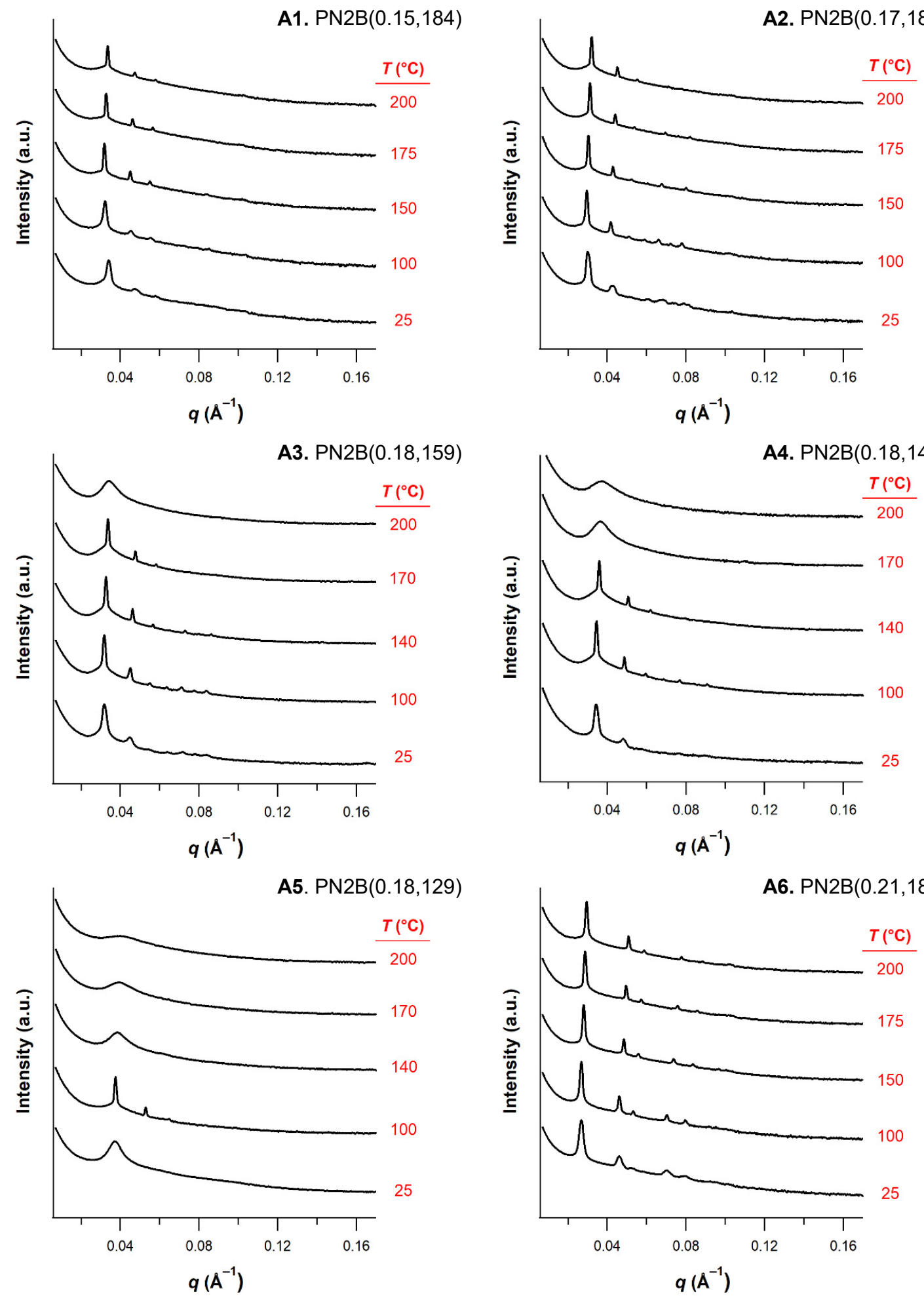
Figure S14 (Page 2/4). 1D azimuthally averaged SAXS patterns for poly(exo,exo-norbornene dimethylester)- $b$-poly(norbornene exo,exo-di- $n$-butylester) (PN2B) block polymers. Samples were annealed as described in the Methods section. Synchrotron-source SAXS data were collected on heating between 25 and $200{ }^{\circ} \mathrm{C}$. Samples were heated to each temperature at $10{ }^{\circ} \mathrm{C} / \mathrm{min}$ and annealed for 15 minutes prior to collecting data.
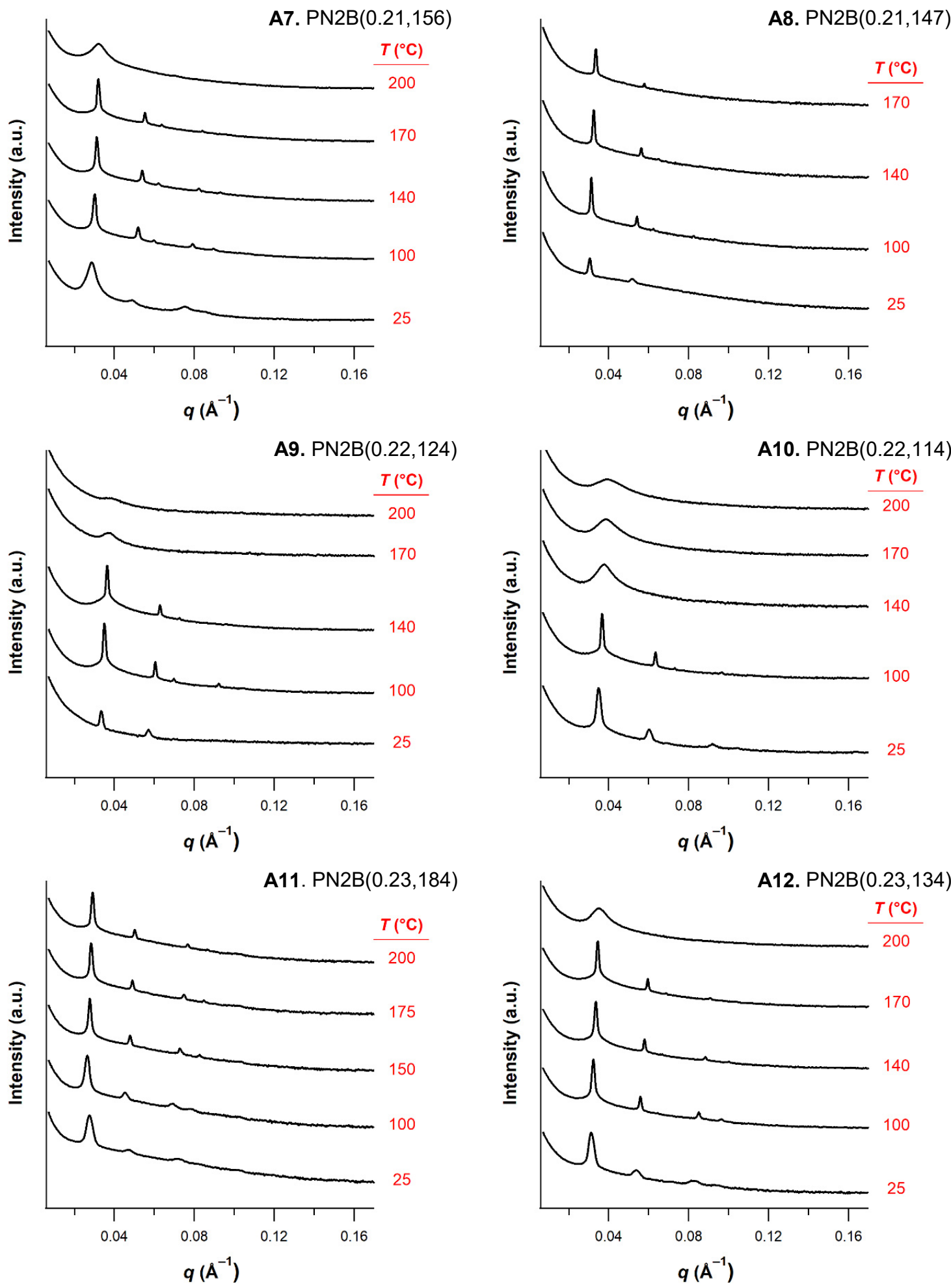
Figure S14 (Page 3/4). 1D azimuthally averaged SAXS patterns for poly(exo,exo-norbornene dimethylester)-b-poly(norbornene exo,exo-di- $n$-butylester) (PN2B) block polymers. Samples were annealed as described in the Methods section. Synchrotron-source SAXS data were collected on heating between 25 and $200{ }^{\circ} \mathrm{C}$. Samples were heated to each temperature at $10^{\circ} \mathrm{C} / \mathrm{min}$ and annealed for 15 minutes prior to collecting data.
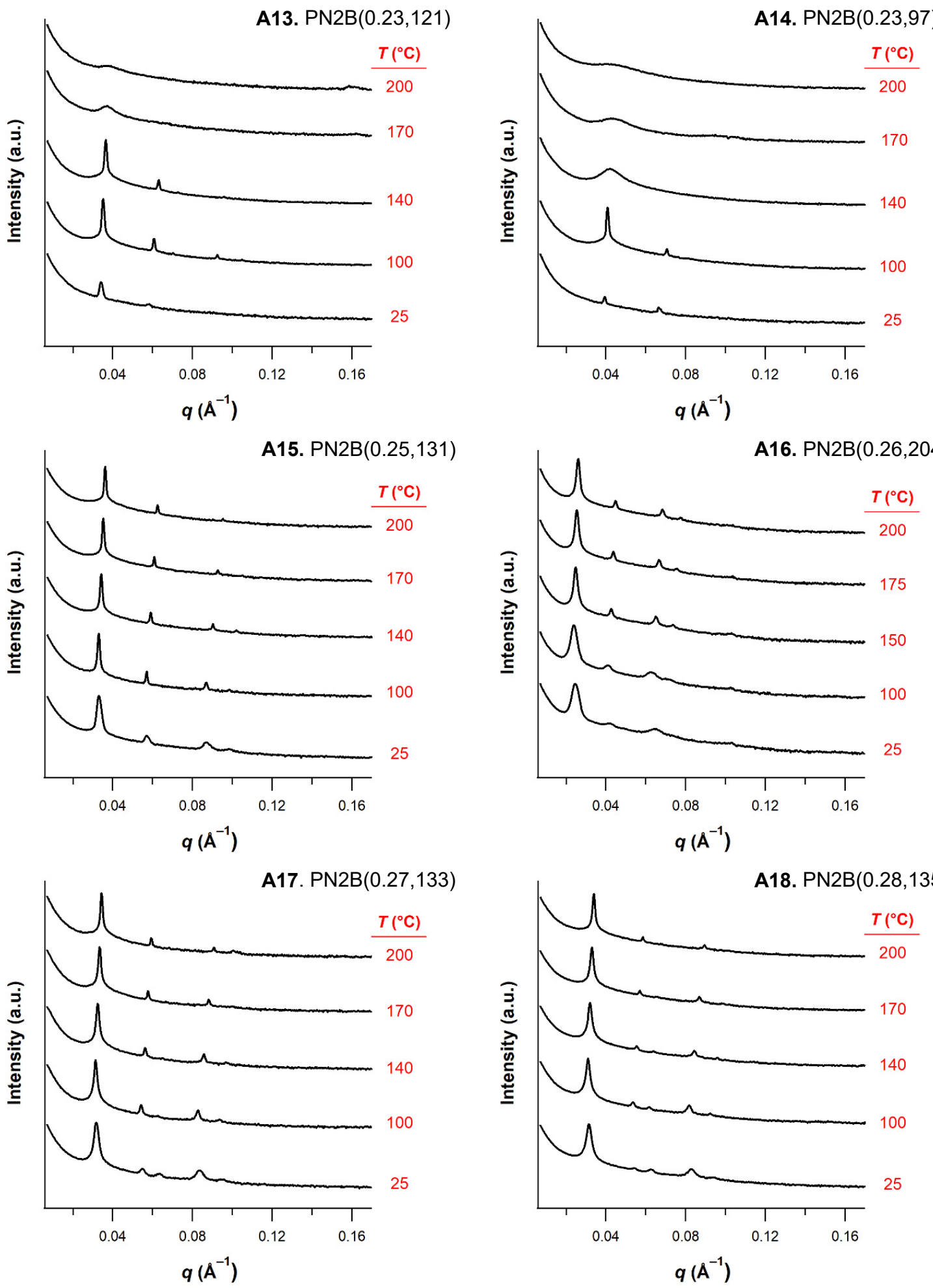
Figure S14 (Page 4/4). 1D azimuthally averaged SAXS patterns for poly(exo,exo-norbornene dimethylester)- $b$-poly(norbornene exo,exo-di- $n$-butylester) (PN2B) block polymers. Samples were annealed as described in the Methods section. Synchrotron-source SAXS data were collected on heating between 25 and $200{ }^{\circ} \mathrm{C}$. Samples were heated to each temperature at $10{ }^{\circ} \mathrm{C} / \mathrm{min}$ and annealed for 15 minutes prior to collecting data. Note: $\mathbf{A 2 0}-\mathbf{A 2 3}$ are disordered at all temperatures and are omitted here.

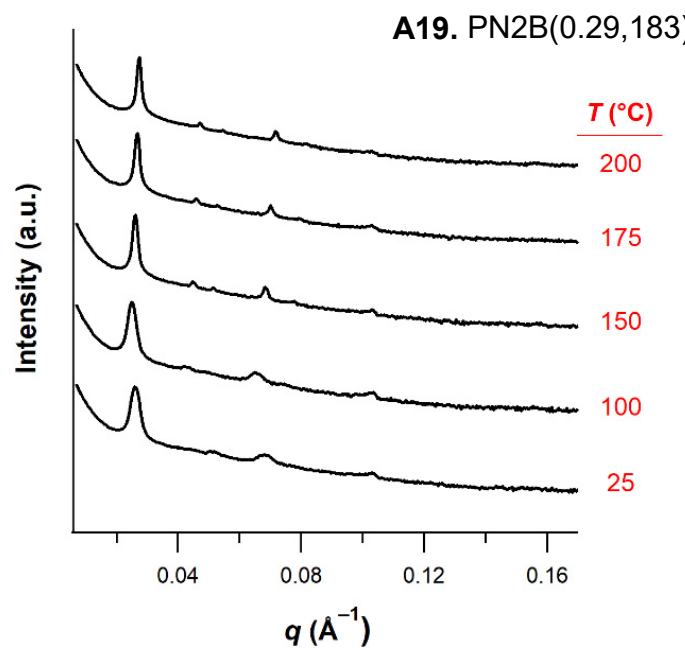


Figure S15 (Page 1/3). 1D azimuthally averaged SAXS patterns for poly(exo,exo-norbornene dimethylester)- $b$-poly(norbornene exo-n-decylimide) (PN1D) block polymers. Samples were annealed as described in the Methods section. Synchrotron-source SAXS data were collected on heating between 25 and $200{ }^{\circ} \mathrm{C}$. Samples were heated to each temperature at $10{ }^{\circ} \mathrm{C} / \mathrm{min}$ and annealed for 15 minutes prior to collecting data.
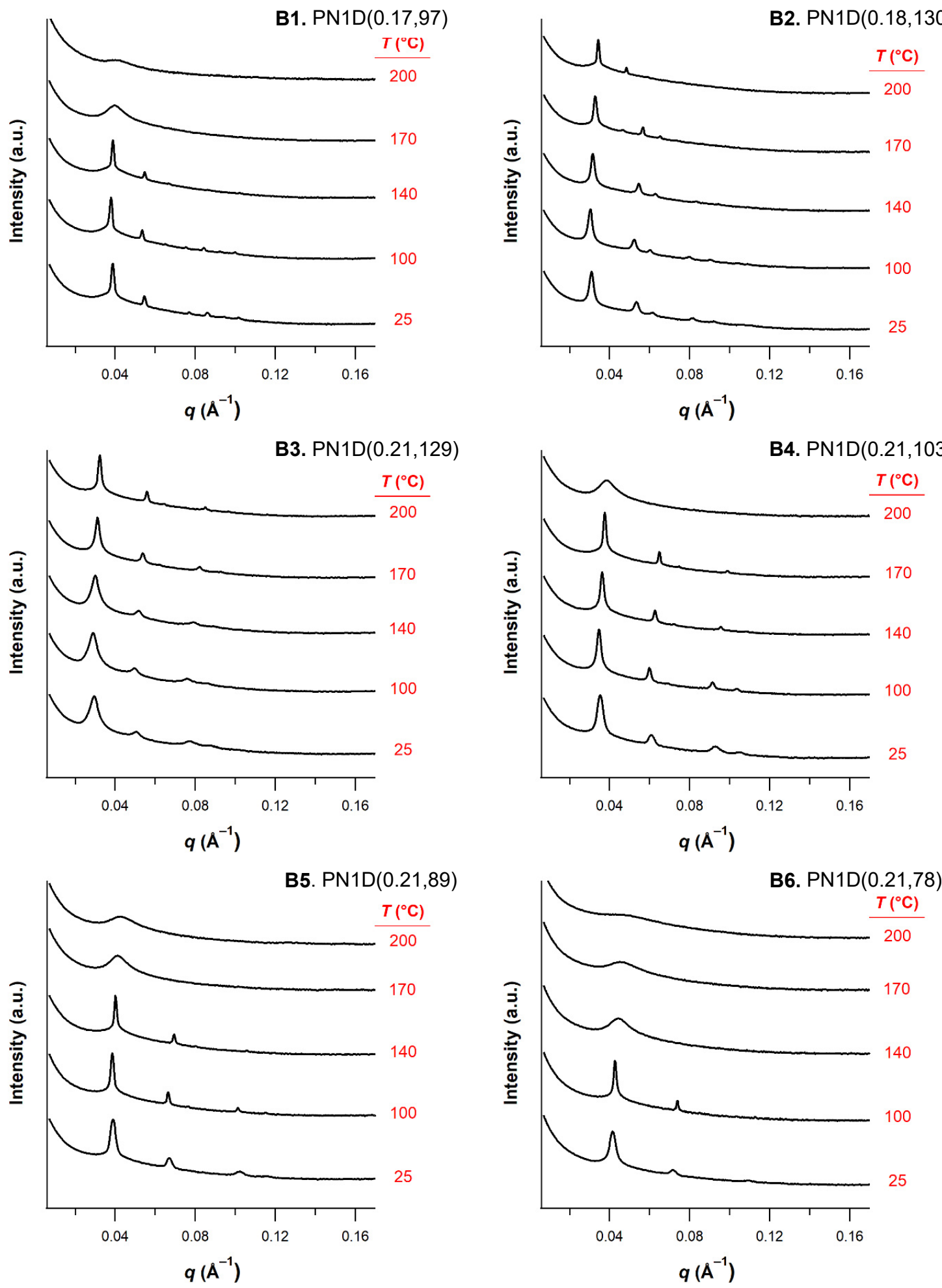
Figure S15 (Page 2/3). 1D azimuthally averaged SAXS patterns for poly(exo,exo-norbornene dimethylester)- $b$-poly(norbornene exo-n-decylimide) (PN1D) block polymers. Samples were annealed as described in the Methods section. Synchrotron-source SAXS data were collected on heating between 25 and $200{ }^{\circ} \mathrm{C}$. Samples were heated to each temperature at $10{ }^{\circ} \mathrm{C} / \mathrm{min}$ and annealed for 15 minutes prior to collecting data.
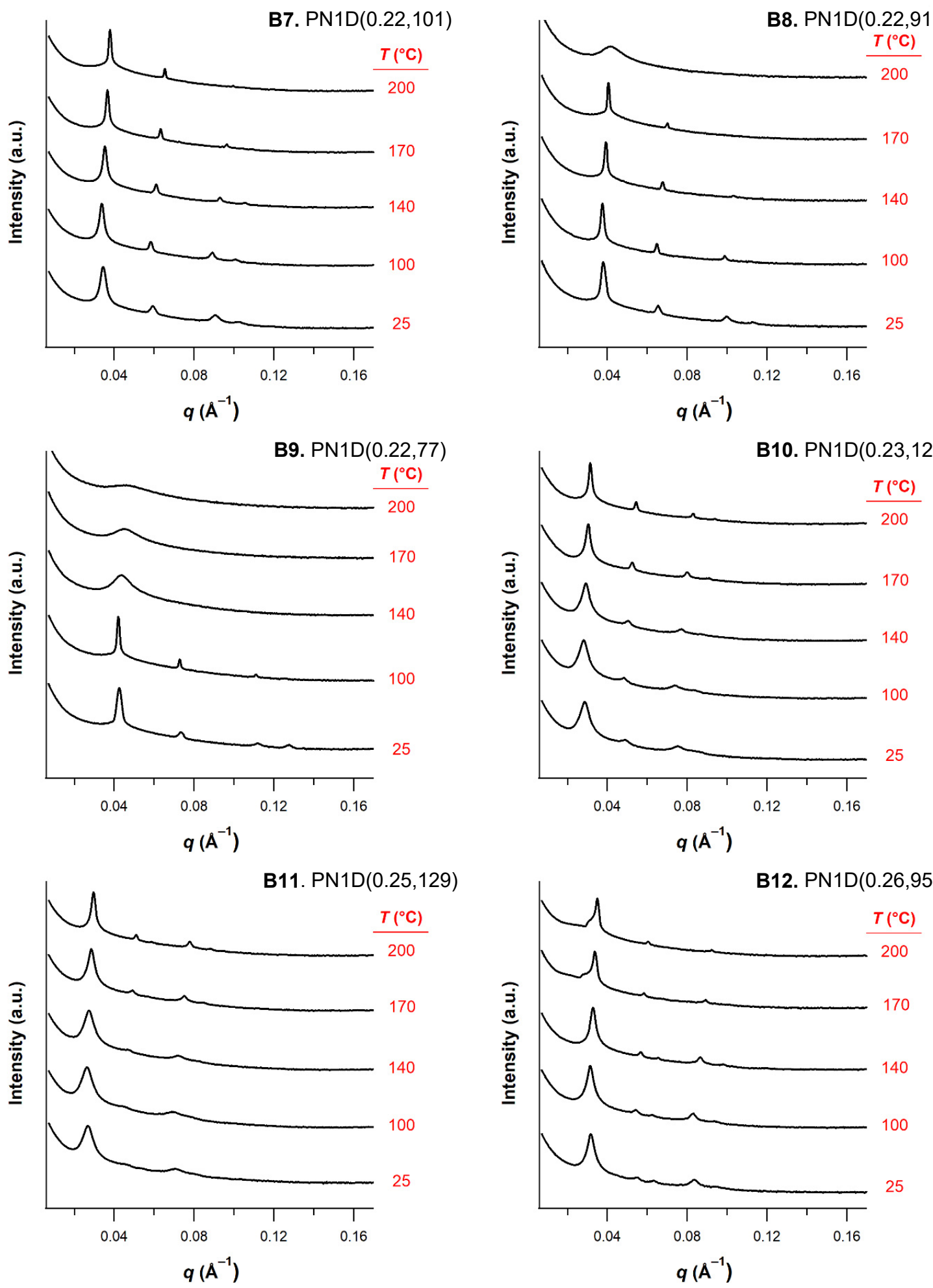
Figure S15 (Page 3/3). 1D azimuthally averaged SAXS patterns for poly(exo,exo-norbornene dimethylester)- $b$-poly(norbornene exo- $n$-decylimide) (PN1D) block polymers. Samples were annealed as described in the Methods section. Synchrotron-source SAXS data were collected on heating between 25 and $200{ }^{\circ} \mathrm{C}$. Samples were heated to each temperature at $10{ }^{\circ} \mathrm{C} / \mathrm{min}$ and annealed for 15 minutes prior to collecting data. Note: B15-B18 are disordered at all temperatures and are omitted here.
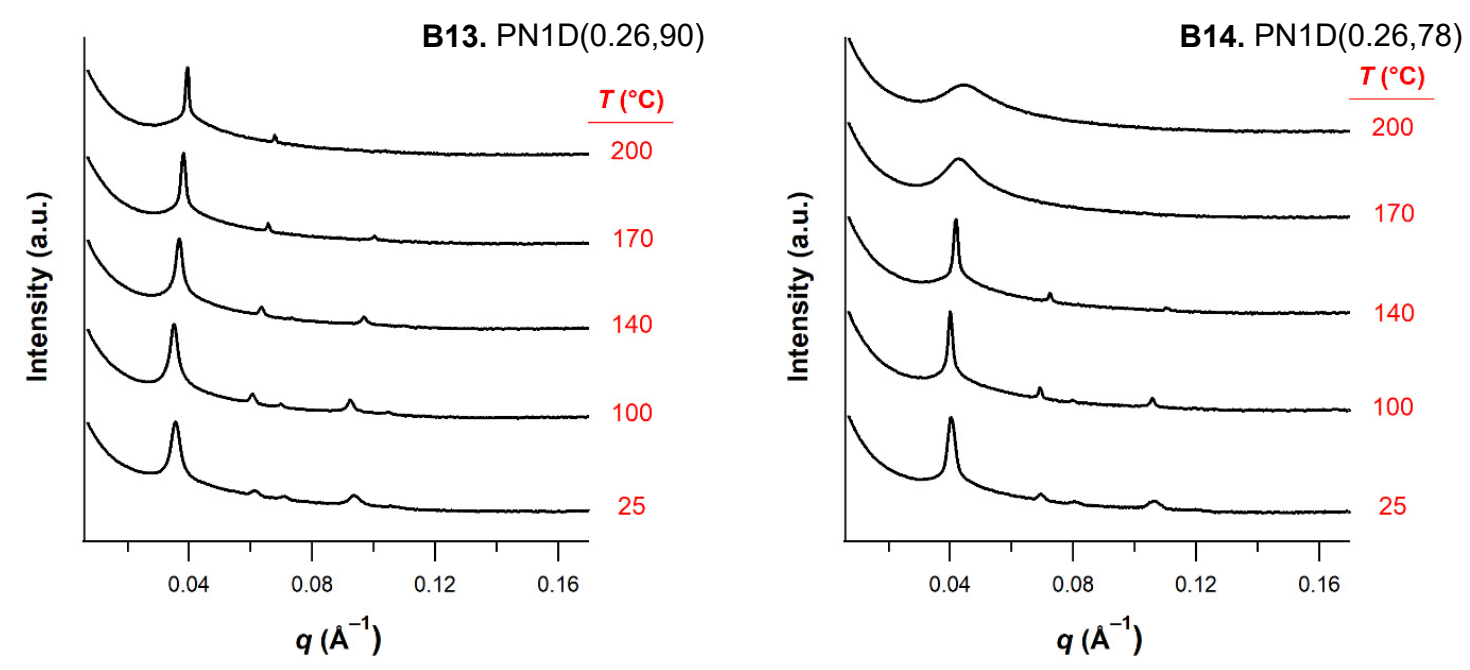
Figure S16 (Page 1/3). 1D azimuthally averaged SAXS patterns for poly(exo,exo-norbornene dimethylester)- $b$-poly(norbornene exo,exo-di- $n$-decylester) (PN2D) block polymers. Samples were annealed as described in the Methods section. Synchrotron-source SAXS data were collected on heating between 25 and $200{ }^{\circ} \mathrm{C}$. Samples were heated to each temperature at $10{ }^{\circ} \mathrm{C} / \mathrm{min}$ and annealed for 15 minutes prior to collecting data.
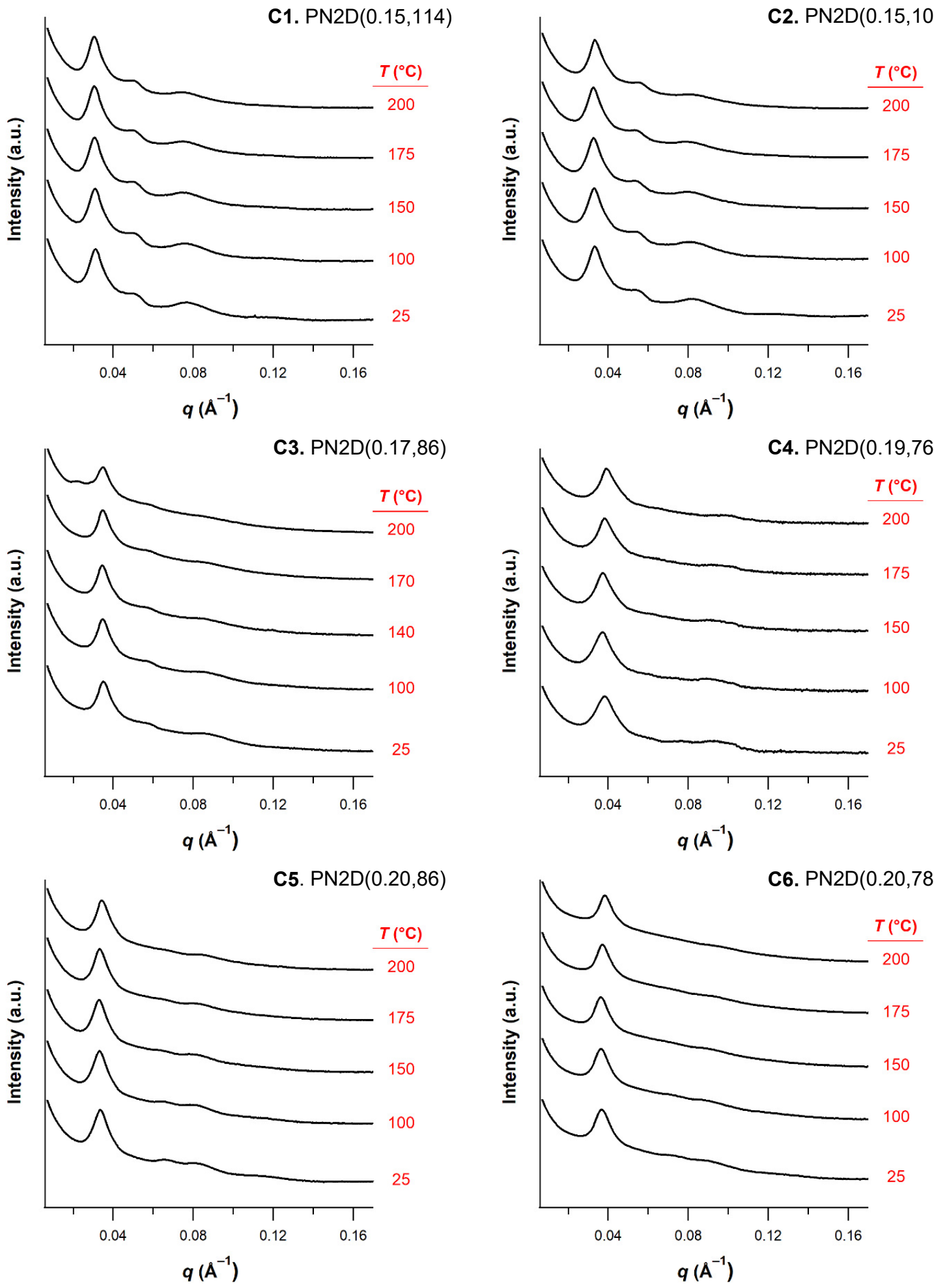
Figure S16 (Page 2/3). 1D azimuthally averaged SAXS patterns for poly(exo,exo-norbornene dimethylester)- $b$-poly(norbornene exo,exo-di- $n$-decylester) (PN2D) block polymers. Samples were annealed as described in the Methods section. Synchrotron-source SAXS data were collected on heating between 25 and $200{ }^{\circ} \mathrm{C}$. Samples were heated to each temperature at $10{ }^{\circ} \mathrm{C} / \mathrm{min}$ and annealed for 15 minutes prior to collecting data.
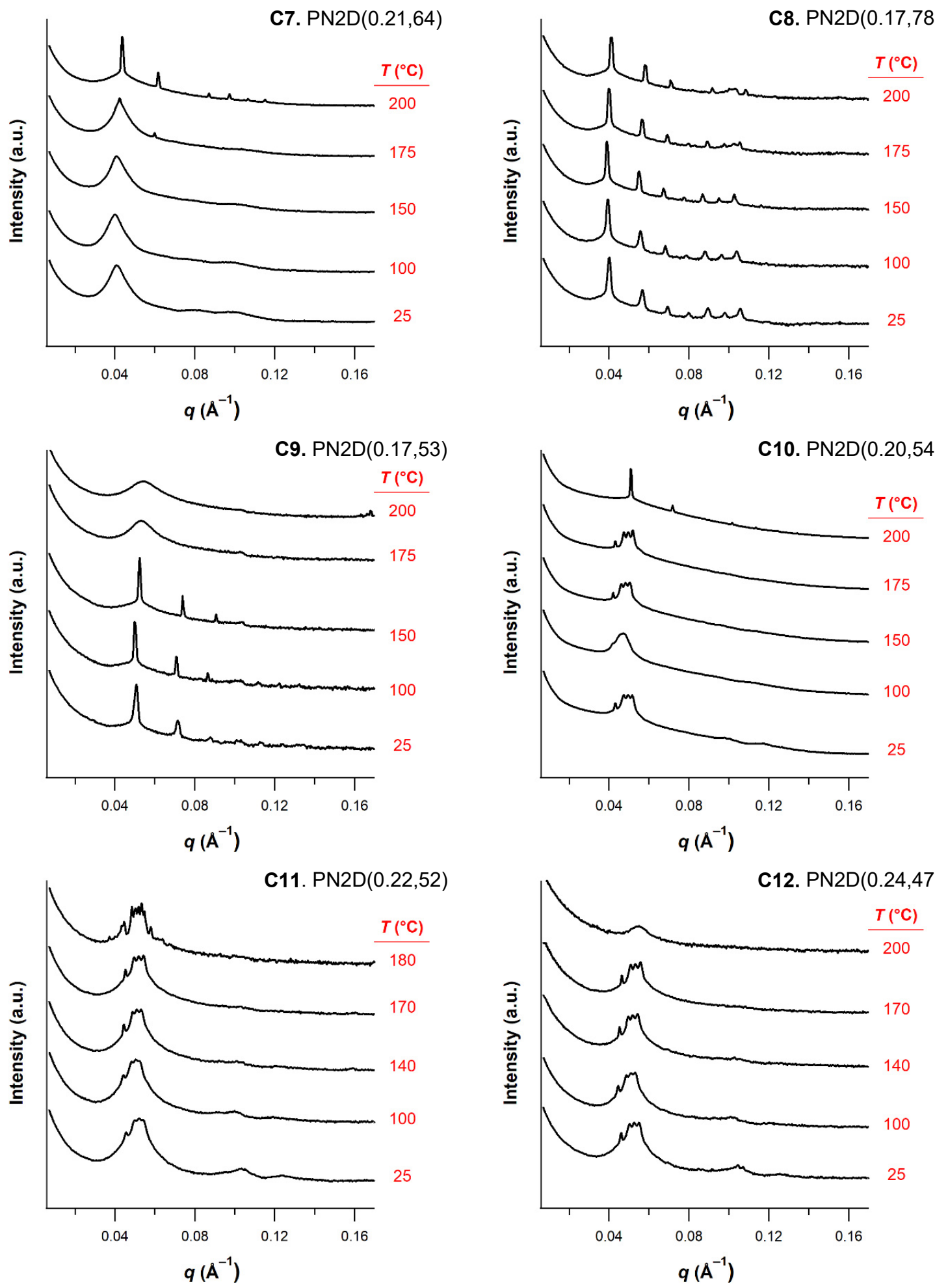
Figure S16 (Page 3/3). 1D azimuthally averaged SAXS patterns for poly(exo,exo-norbornene dimethylester)- $b$-poly(norbornene exo,exo-di- $n$-decylester) (PN2D) block polymers. Samples were annealed as described in the Methods section. Synchrotron-source SAXS data were collected on heating between 25 and $200{ }^{\circ} \mathrm{C}$. Samples were heated to each temperature at $10{ }^{\circ} \mathrm{C} / \mathrm{min}$ and annealed for 15 minutes prior to collecting data.
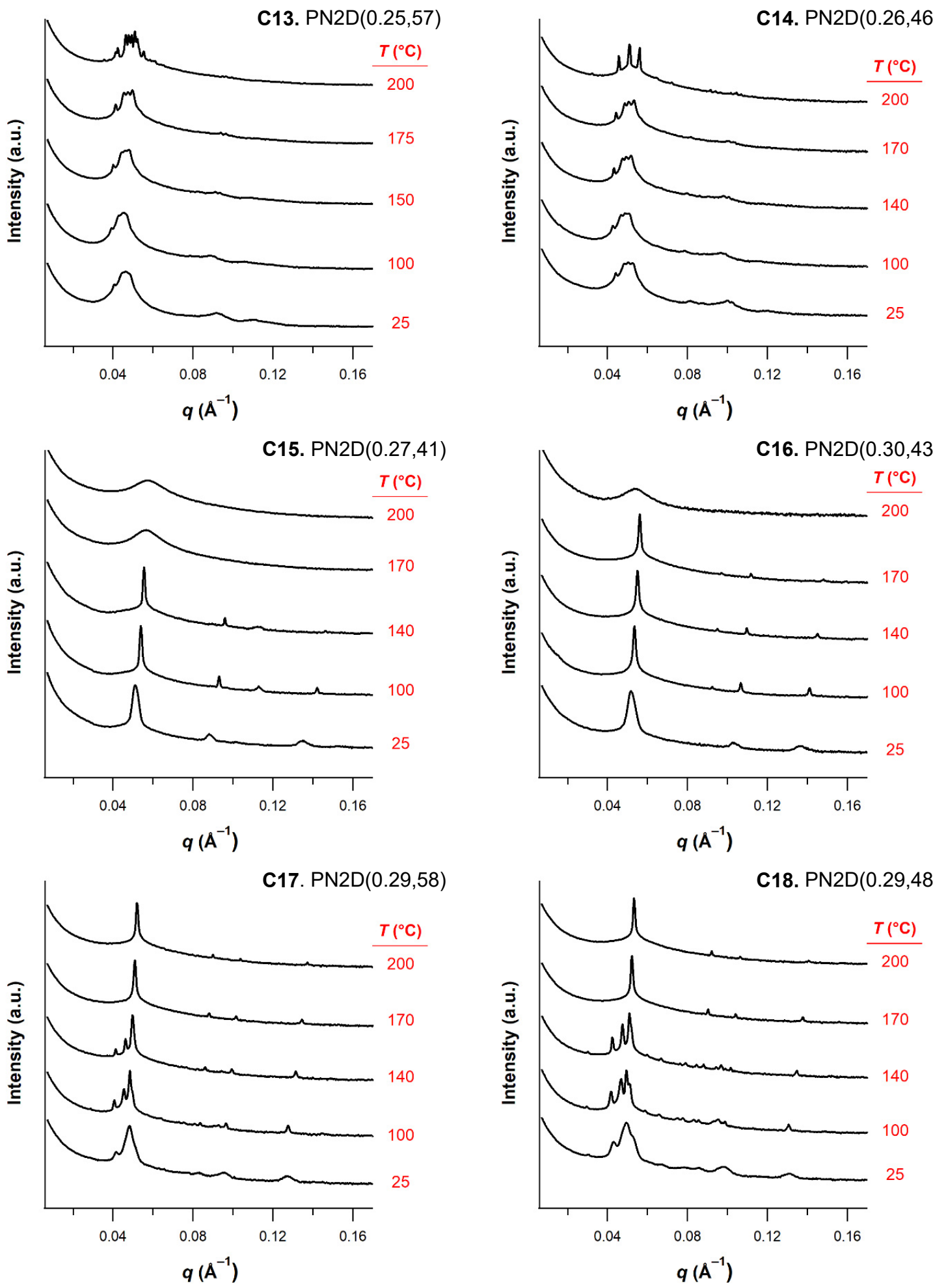
Figure S17 (Page 1/9). 1D azimuthally averaged SAXS patterns for poly(exo,exo-norbornene dimethylester)- $b$-poly(norbornene exo,exo-di- $n$-decylester) (PN2D) block polymers. Samples were not annealed before measurement. Synchrotron-source SAXS data were collected on heating between 25 and $200{ }^{\circ} \mathrm{C}$. Samples were heated to each temperature at $10{ }^{\circ} \mathrm{C} / \mathrm{min}$ and annealed for 15 minutes prior to collecting data.
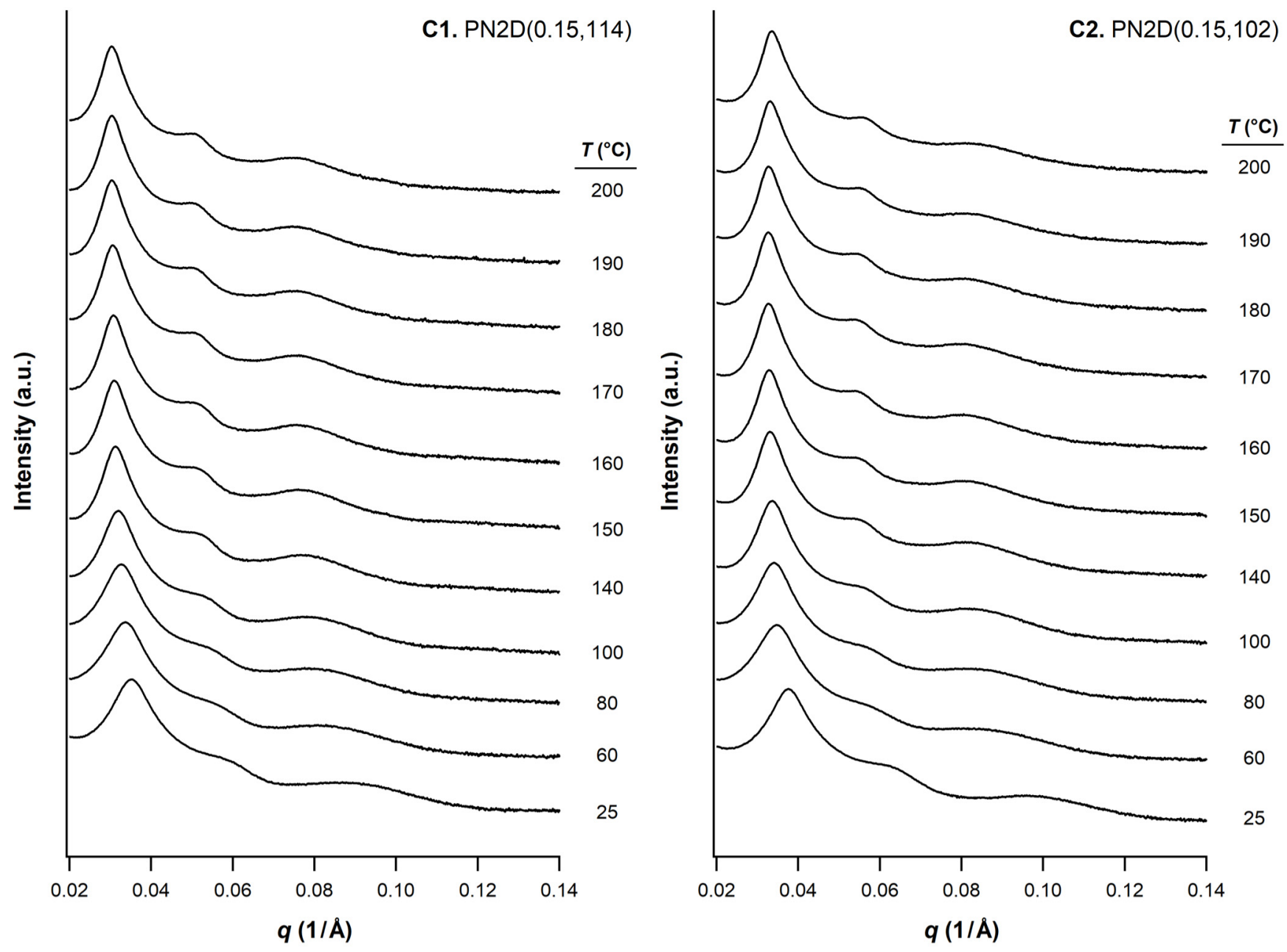
Figure S17 (Page 2/9). 1D azimuthally averaged SAXS patterns for poly(exo,exo-norbornene dimethylester)- $b$-poly(norbornene exo,exo-di- $n$-decylester) (PN2D) block polymers. Samples were not annealed before measurement. Synchrotron-source SAXS data were collected on heating between 25 and $200{ }^{\circ} \mathrm{C}$. Samples were heated to each temperature at $10{ }^{\circ} \mathrm{C} / \mathrm{min}$ and annealed for 15 minutes prior to collecting data.
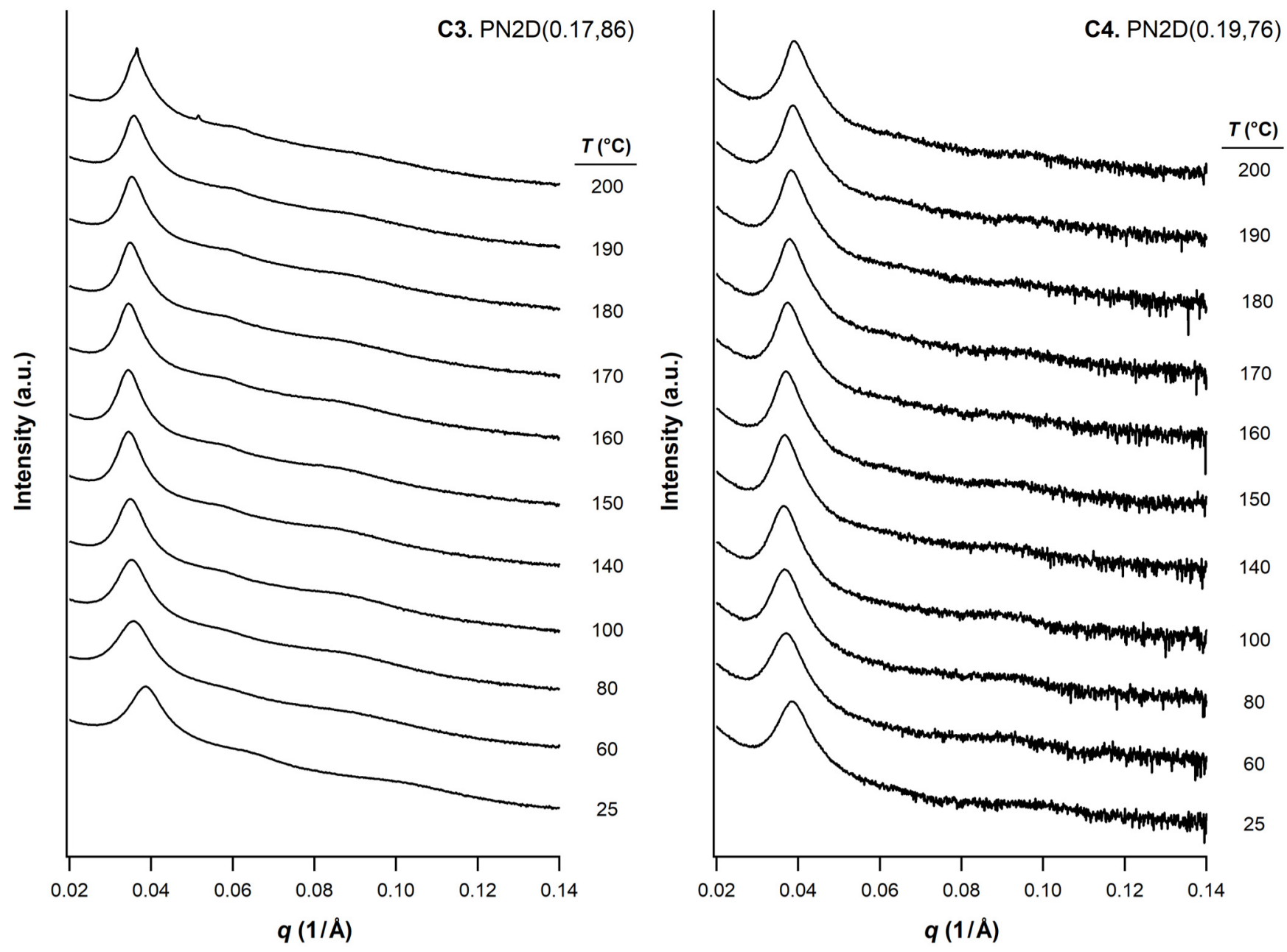
Figure S17 (Page 3/9). 1D azimuthally averaged SAXS patterns for poly(exo,exo-norbornene dimethylester)- $b$-poly(norbornene exo,exo-di- $n$-decylester) (PN2D) block polymers. Samples were not annealed before measurement. Synchrotron-source SAXS data were collected on heating between 25 and $200{ }^{\circ} \mathrm{C}$. Samples were heated to each temperature at $10{ }^{\circ} \mathrm{C} / \mathrm{min}$ and annealed for 15 minutes prior to collecting data.
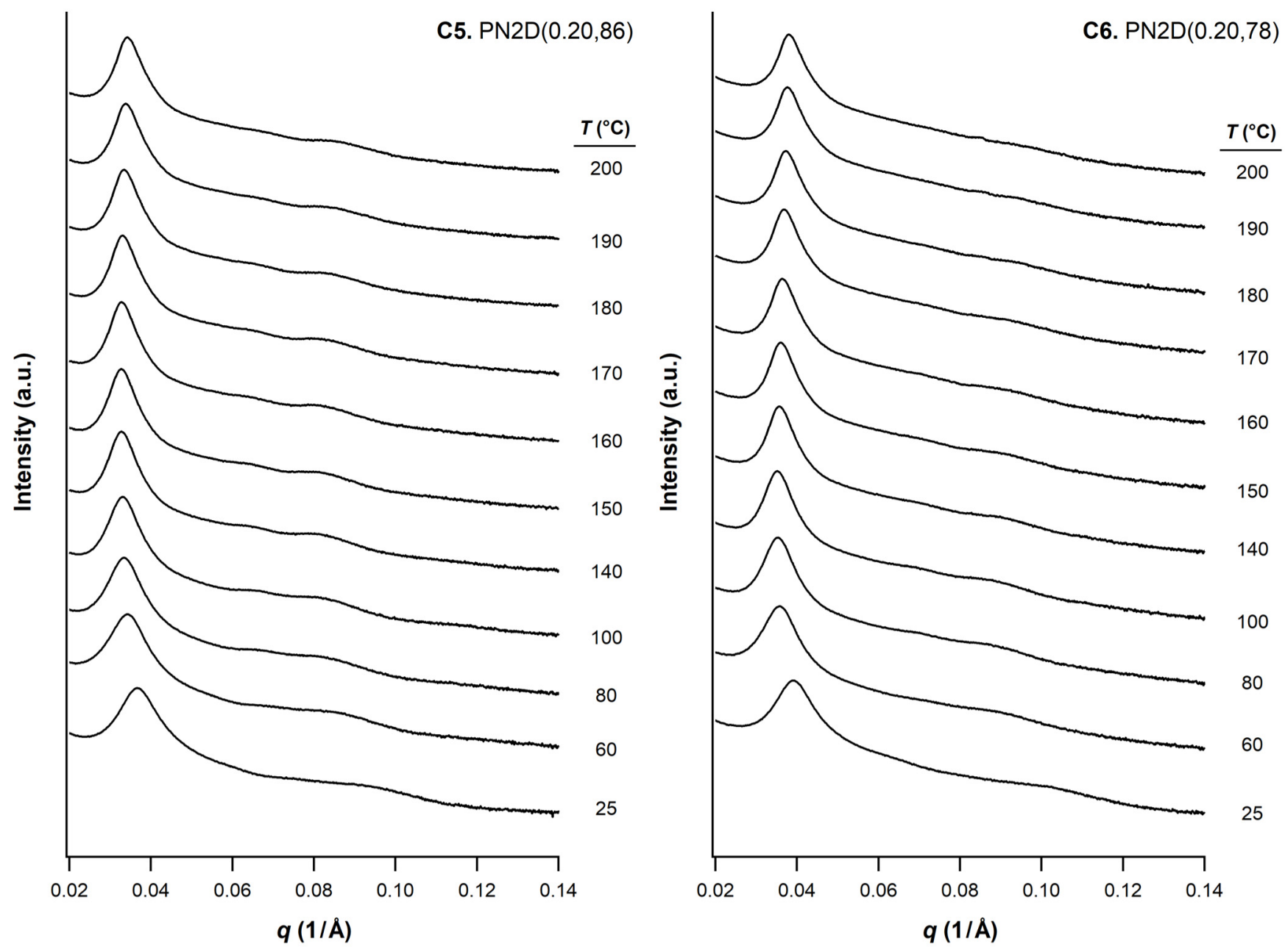
Figure S17 (Page 4/9). 1D azimuthally averaged SAXS patterns for poly(exo,exo-norbornene dimethylester)- $b$-poly(norbornene exo,exo-di- $n$-decylester) (PN2D) block polymers. Samples were not annealed before measurement. Synchrotron-source SAXS data were collected on heating between 25 and $200{ }^{\circ} \mathrm{C}$. Samples were heated to each temperature at $10{ }^{\circ} \mathrm{C} / \mathrm{min}$ and annealed for 15 minutes prior to collecting data.
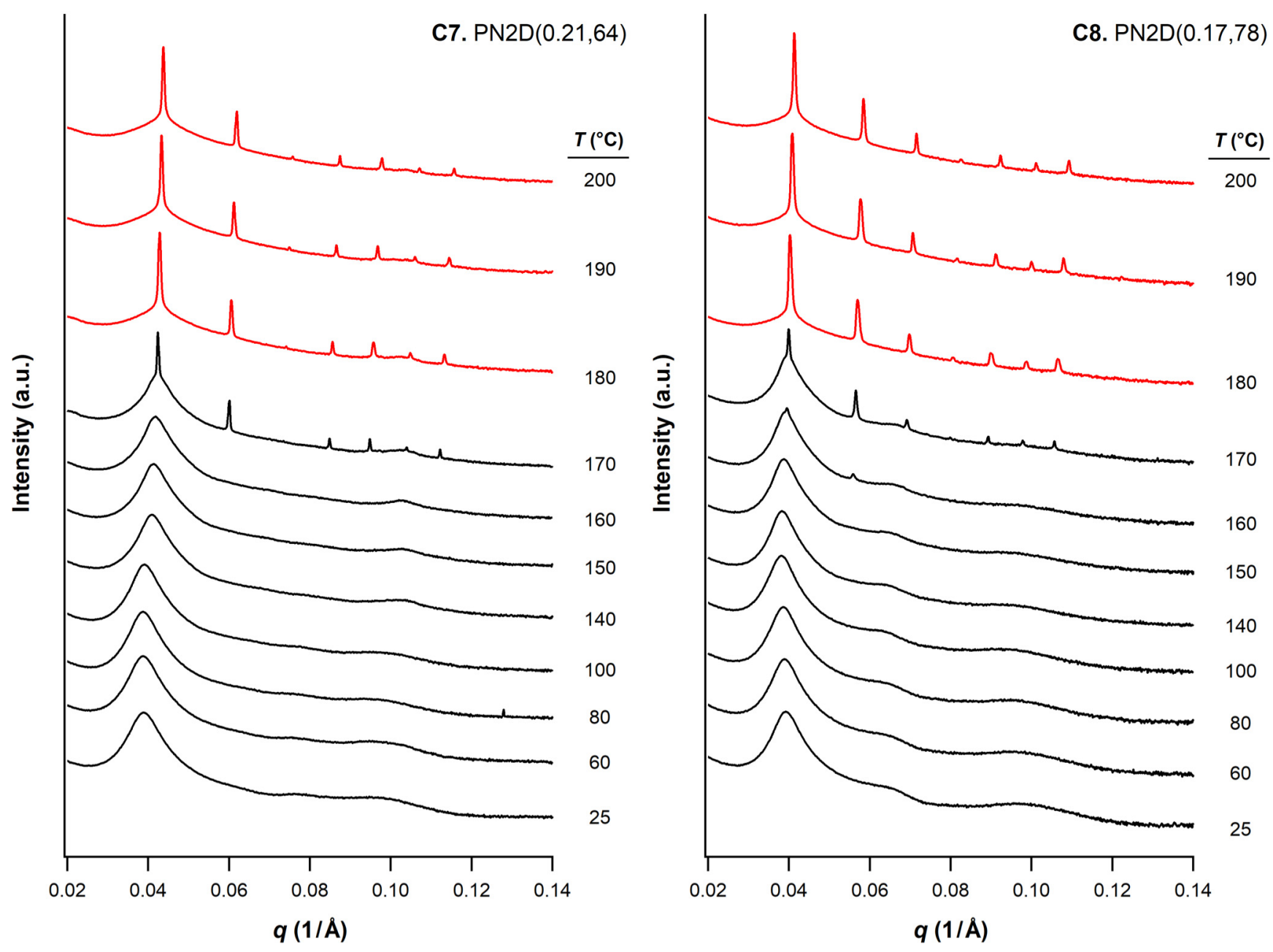
Figure S17 (Page 5/9). 1D azimuthally averaged SAXS patterns for poly(exo,exo-norbornene dimethylester)- $b$-poly(norbornene exo,exo-di- $n$-decylester) (PN2D) block polymers. Samples were not annealed before measurement. Synchrotron-source SAXS data were collected on heating between 25 and $200{ }^{\circ} \mathrm{C}$. Samples were heated to each temperature at $10{ }^{\circ} \mathrm{C} / \mathrm{min}$ and annealed for 15 minutes prior to collecting data.
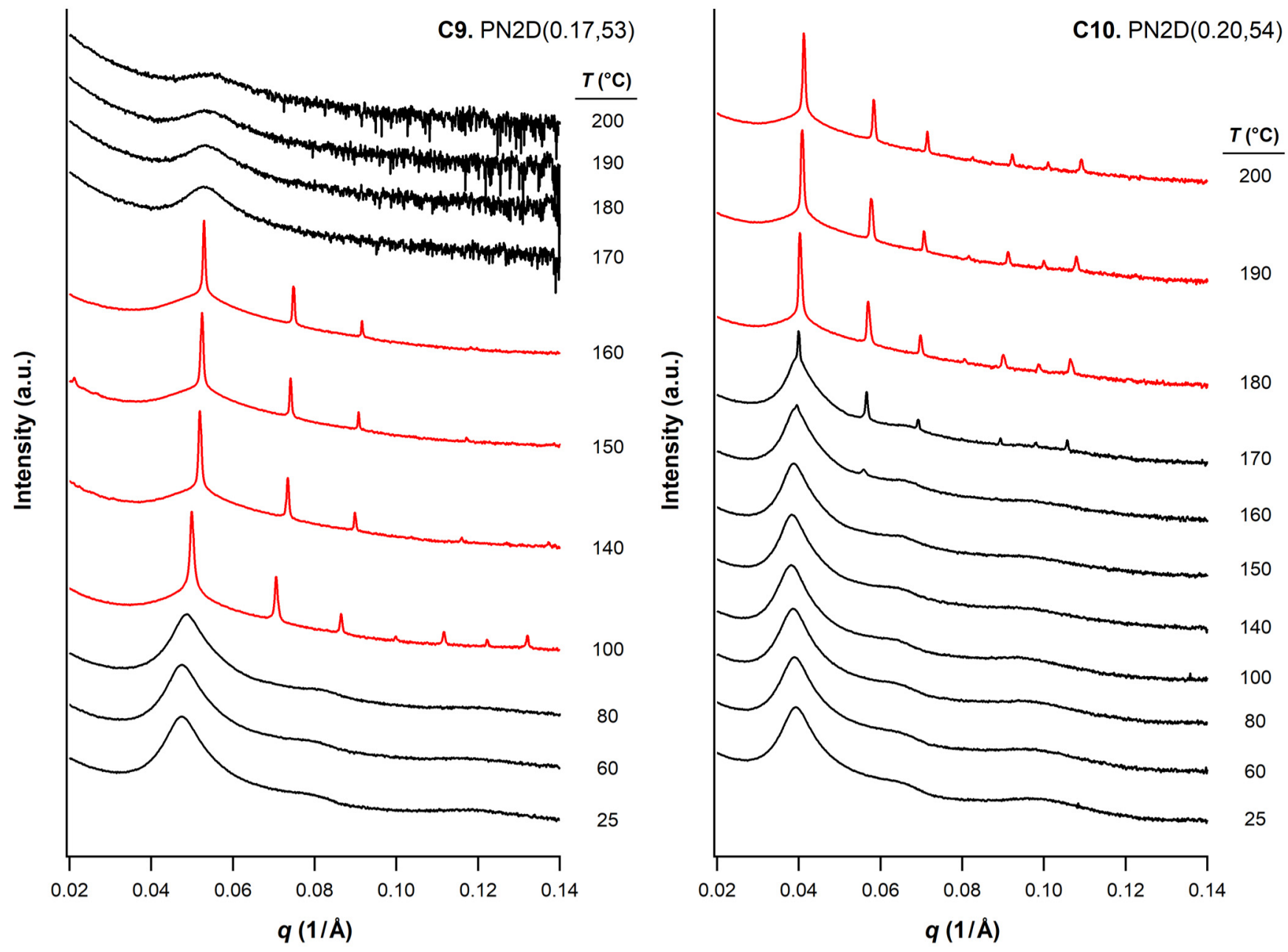
Figure S17 (Page 6/9). 1D azimuthally averaged SAXS patterns for poly(exo,exo-norbornene dimethylester)- $b$-poly(norbornene exo,exo-di- $n$-decylester) (PN2D) block polymers. Samples were not annealed before measurement. Synchrotron-source SAXS data were collected on heating between 25 and $200{ }^{\circ} \mathrm{C}$. Samples were heated to each temperature at $10{ }^{\circ} \mathrm{C} / \mathrm{min}$ and annealed for 15 minutes prior to collecting data.
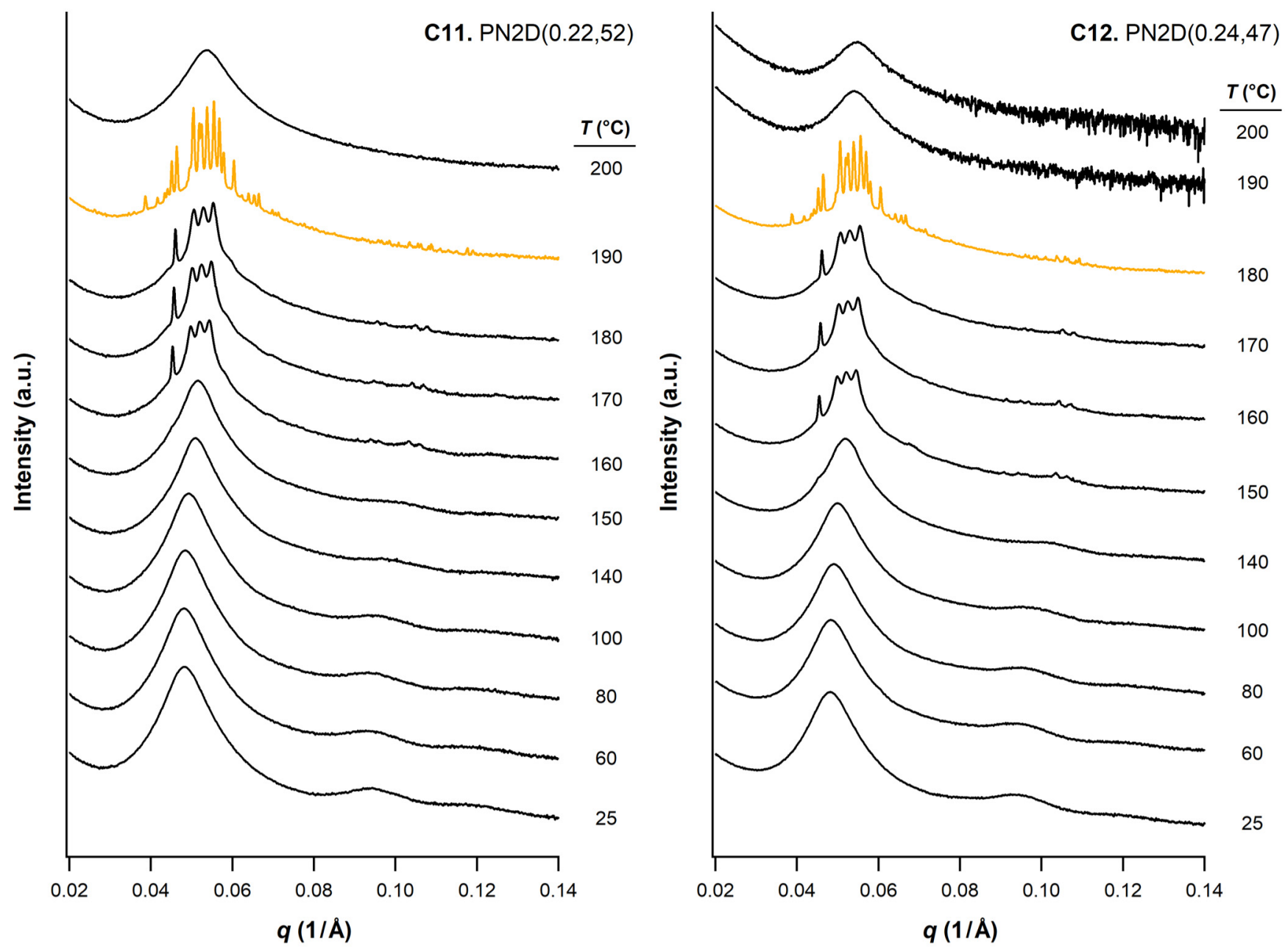
Figure S17 (Page 7/9). 1D azimuthally averaged SAXS patterns for poly(exo,exo-norbornene dimethylester)- $b$-poly(norbornene exo,exo-di- $n$-decylester) (PN2D) block polymers. Samples were not annealed before measurement. Synchrotron-source SAXS data were collected on heating between 25 and $200{ }^{\circ} \mathrm{C}$. Samples were heated to each temperature at $10{ }^{\circ} \mathrm{C} / \mathrm{min}$ and annealed for 15 minutes prior to collecting data.
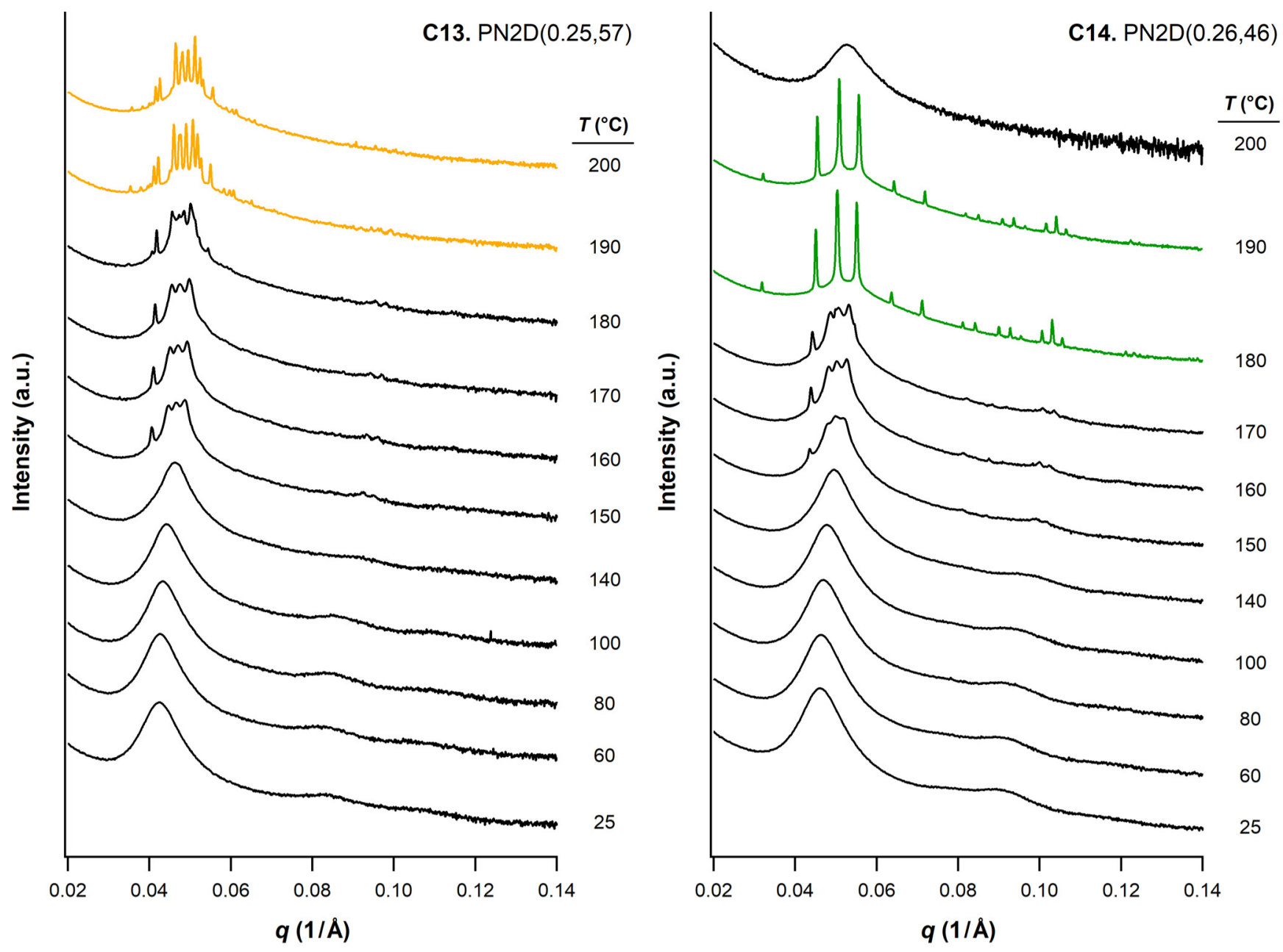
Figure S17 (Page 8/9). 1D azimuthally averaged SAXS patterns for poly(exo,exo-norbornene dimethylester)- $b$-poly(norbornene exo,exo-di- $n$-decylester) (PN2D) block polymers. Samples were not annealed before measurement. Synchrotron-source SAXS data were collected on heating between 25 and $200{ }^{\circ} \mathrm{C}$. Samples were heated to each temperature at $10{ }^{\circ} \mathrm{C} / \mathrm{min}$ and annealed for 15 minutes prior to collecting data.
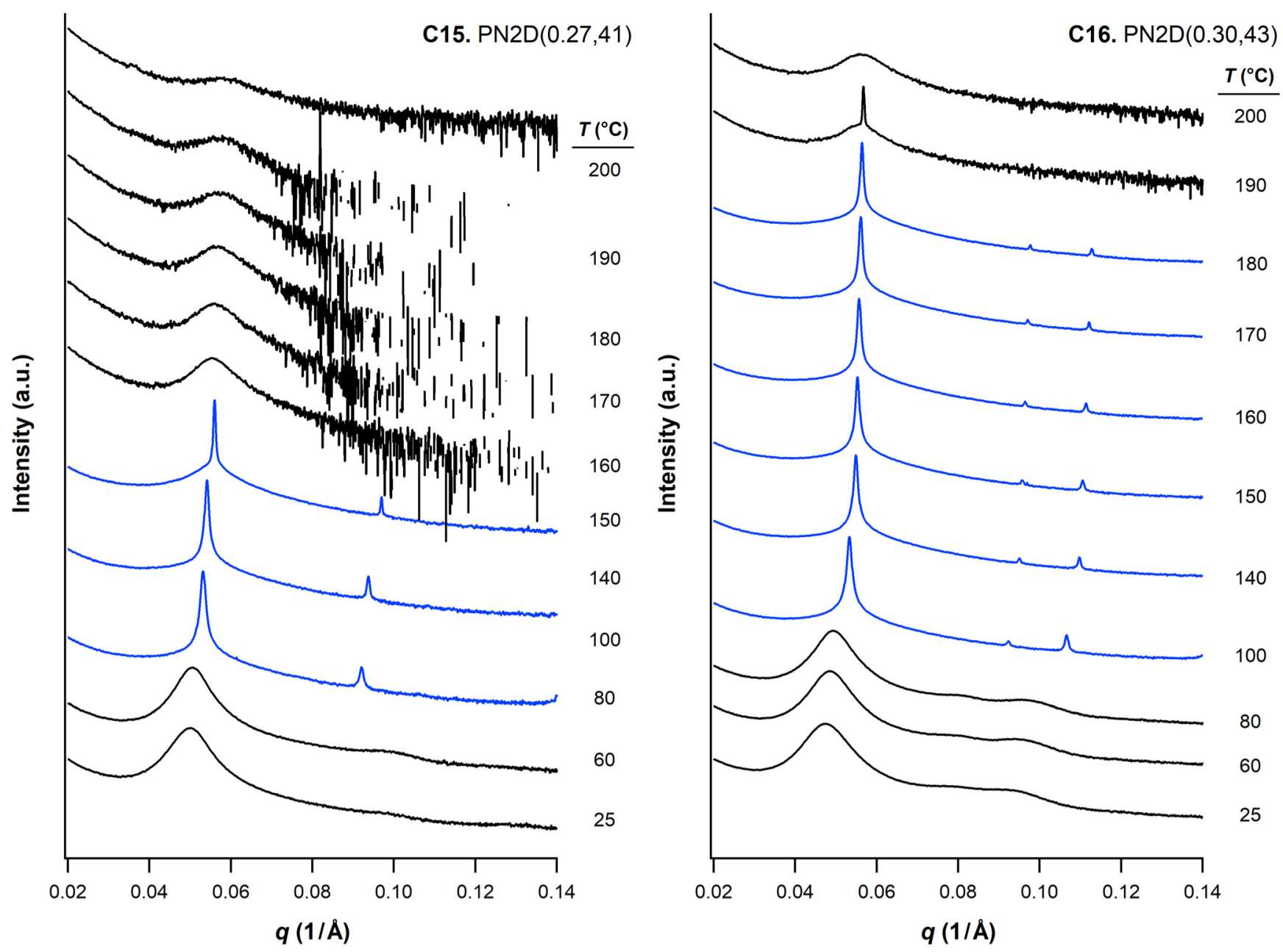
Figure S17 (Page 9/9). 1D azimuthally averaged SAXS patterns for poly(exo,exo-norbornene dimethylester)- $b$-poly(norbornene exo,exo-di- $n$-decylester) (PN2D) block polymers. Samples were not annealed before measurement. Synchrotron-source SAXS data were collected on heating between 25 and $200{ }^{\circ} \mathrm{C}$. Samples were heated to each temperature at $10{ }^{\circ} \mathrm{C} / \mathrm{min}$ and annealed for 15 minutes prior to collecting data.
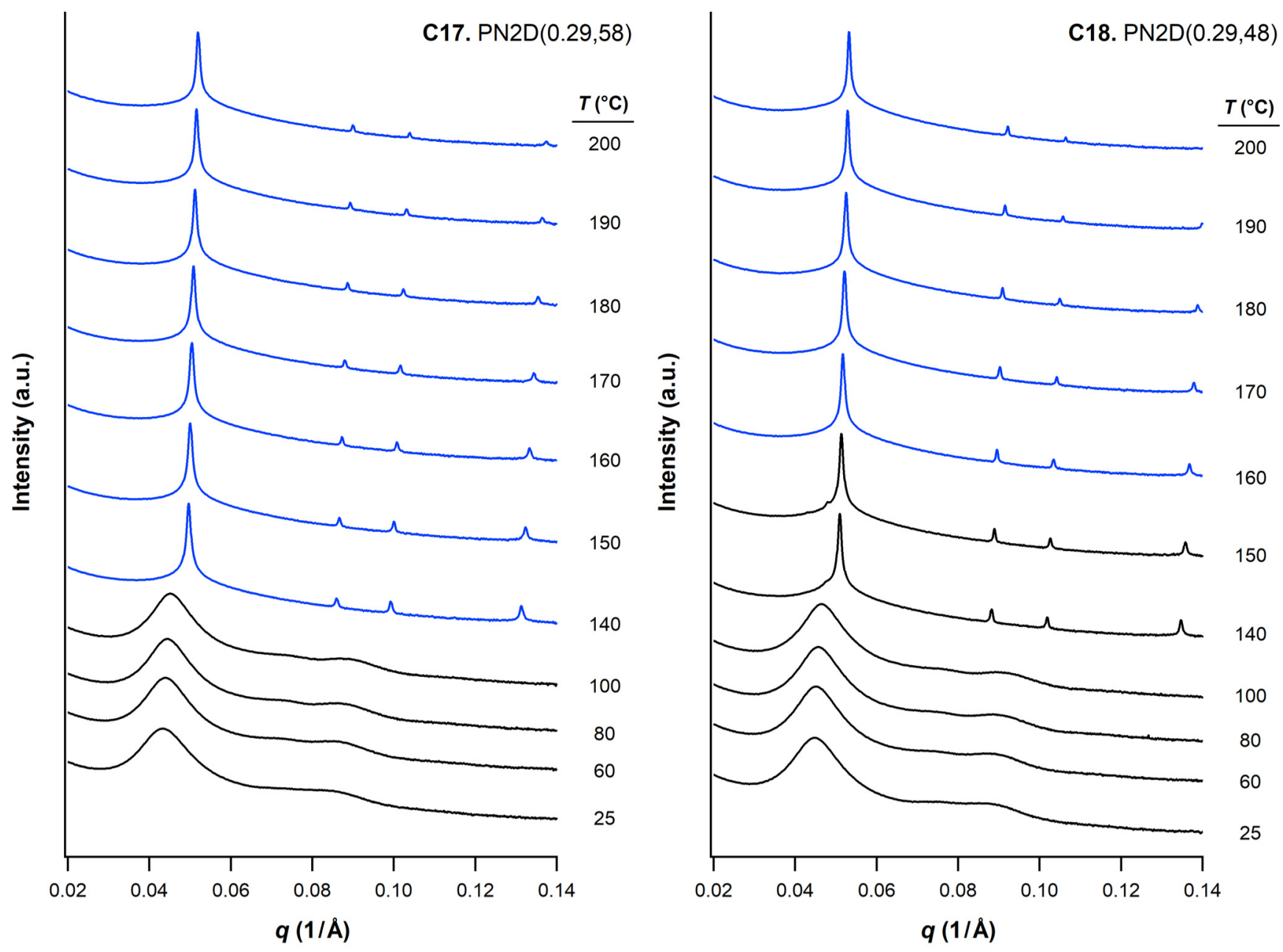


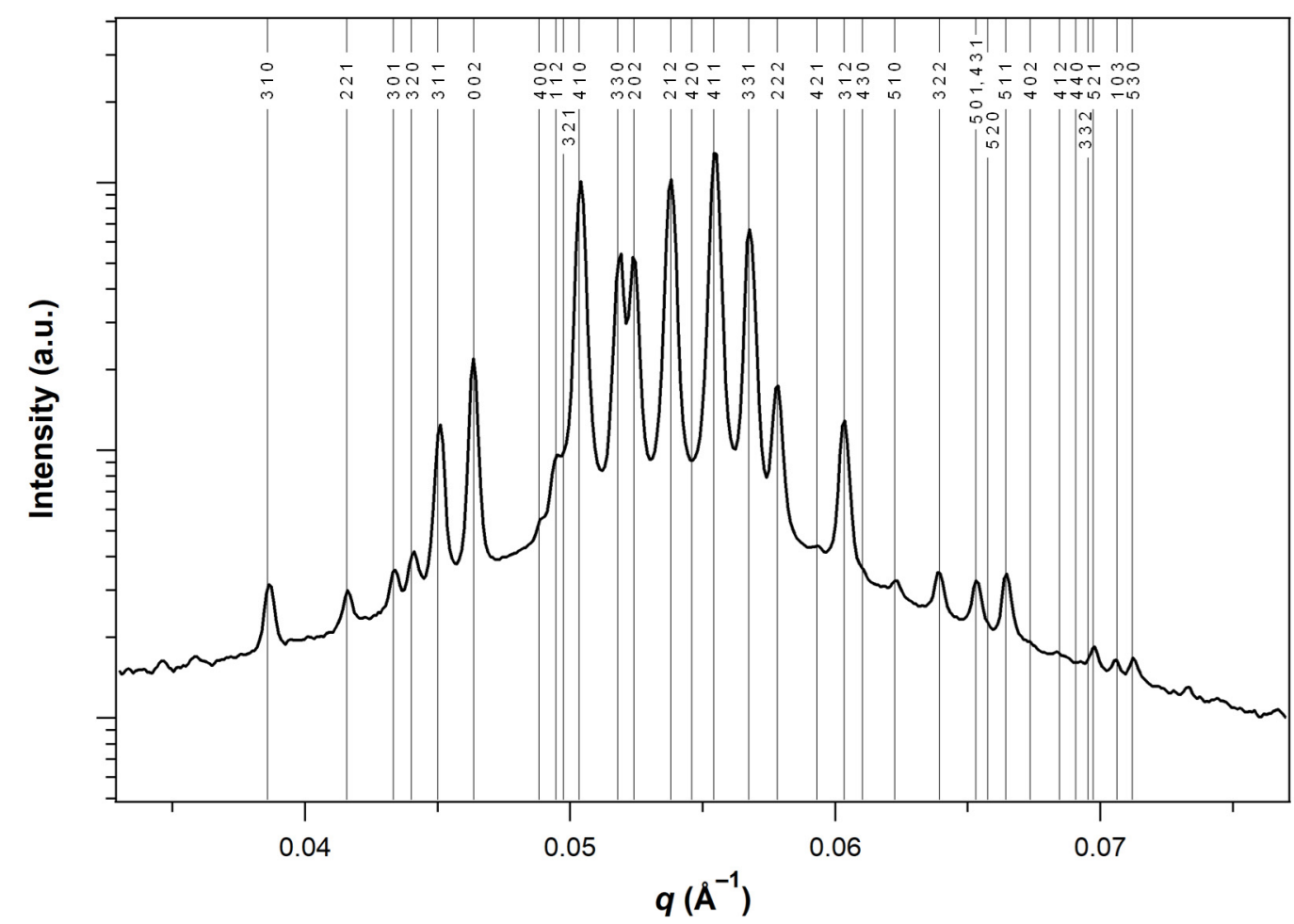

Frank-Kasper $\sigma$

$\mathrm{PH}_{2} / \mathrm{mnm}$

$a=514.5, c=270.9 \AA$

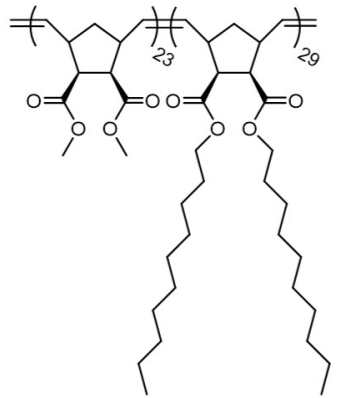

C11. $\mathrm{PN} 2 \mathrm{D}(0.22,52)$

Figure S18. 1D azimuthally averaged SAXS pattern for C11. PN2D $(0.22,52)$. The data were obtained at $190{ }^{\circ} \mathrm{C}$ upon direct heating from the unannealed, freeze-dried state. Vertical lines represent calculated reflections for the Frank-Kasper $\sigma$ phase (tetragonal space group, $P 4_{2} / \mathrm{mnm}$ ). 


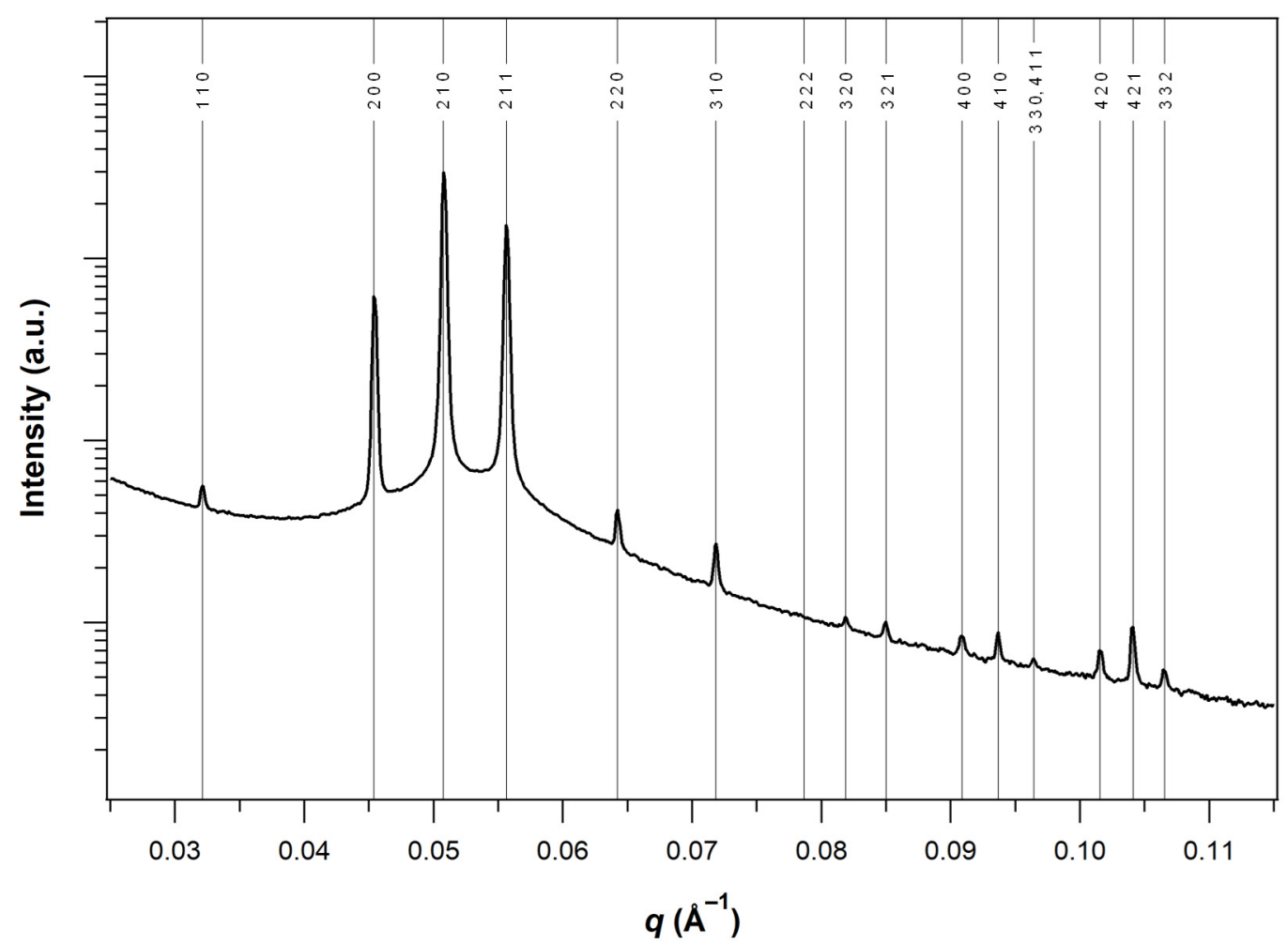

Frank-Kasper A15

$\operatorname{Pm} \overline{3} n$

$a=276.6 \AA$

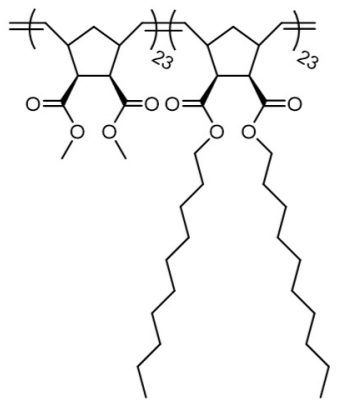

C14. $\mathrm{PN} 2 \mathrm{D}(0.26,46)$

Figure S19. 1D azimuthally averaged SAXS pattern for C14. PN2D $(0.26,46)$. The data were obtained at $190{ }^{\circ} \mathrm{C}$ upon direct heating from the unannealed, freeze-dried state. Vertical lines represent calculated reflections for the Frank-Kasper A15 phase (cubic space group, $\boldsymbol{P} \boldsymbol{m} \overline{\mathbf{3}} \boldsymbol{n}$ ). 


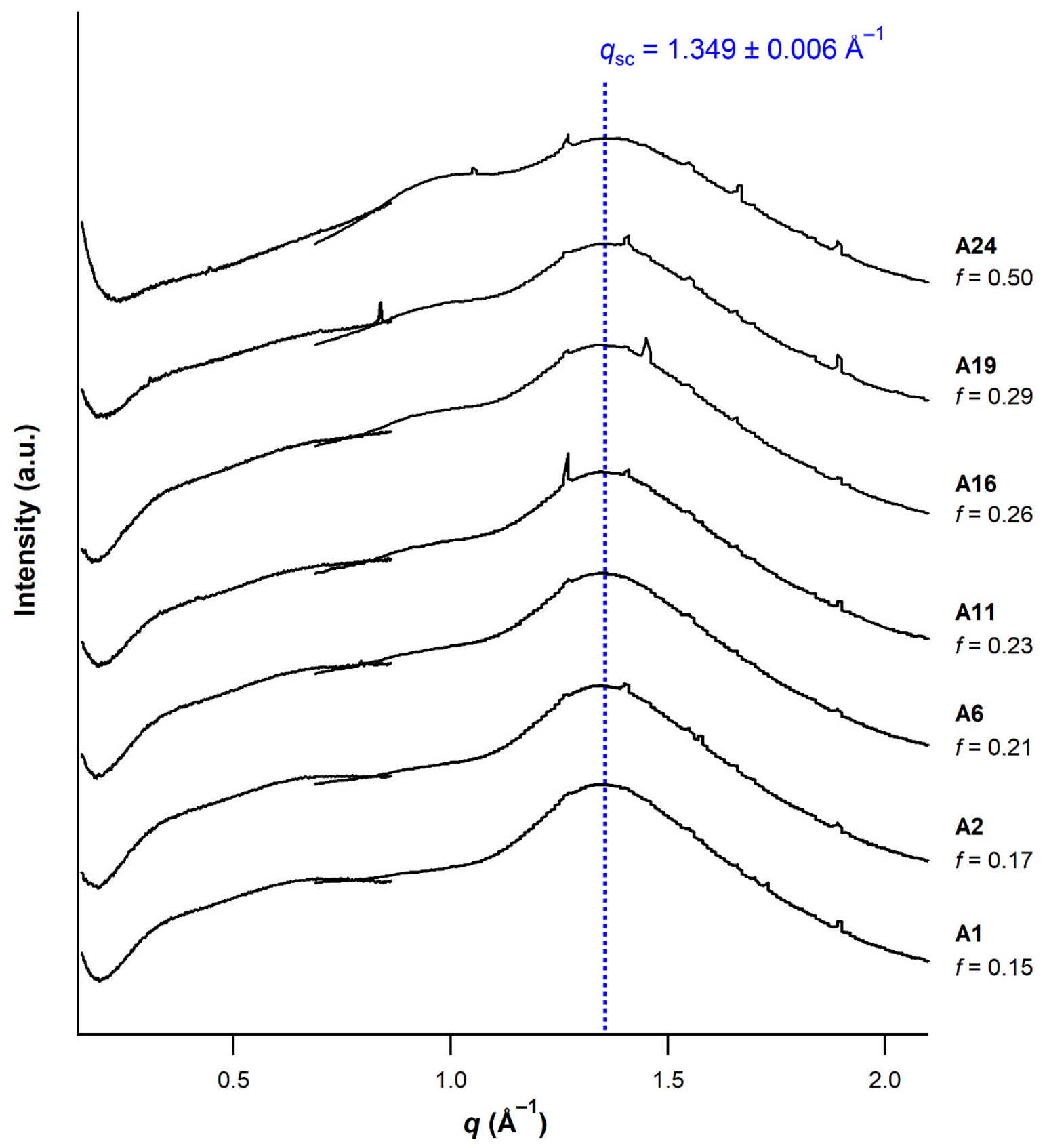

Figure S20. 1D-averaged mid- and wide-angle X-ray scattering (MAXS and WAXS) data for select PN2M- $b$-PN2B block polymers at $25^{\circ} \mathrm{C}$. Molecular characterization data for all samples is provided in Table S1. No peak corresponding to $q$ bb is observed in the MAXS (lower- $q$ ) range. In the WAXS range, $q_{\mathrm{sc}}=1.349 \pm 0.006 \AA^{-1}$ corresponds to the average distance between $n$-butyl side chains. 


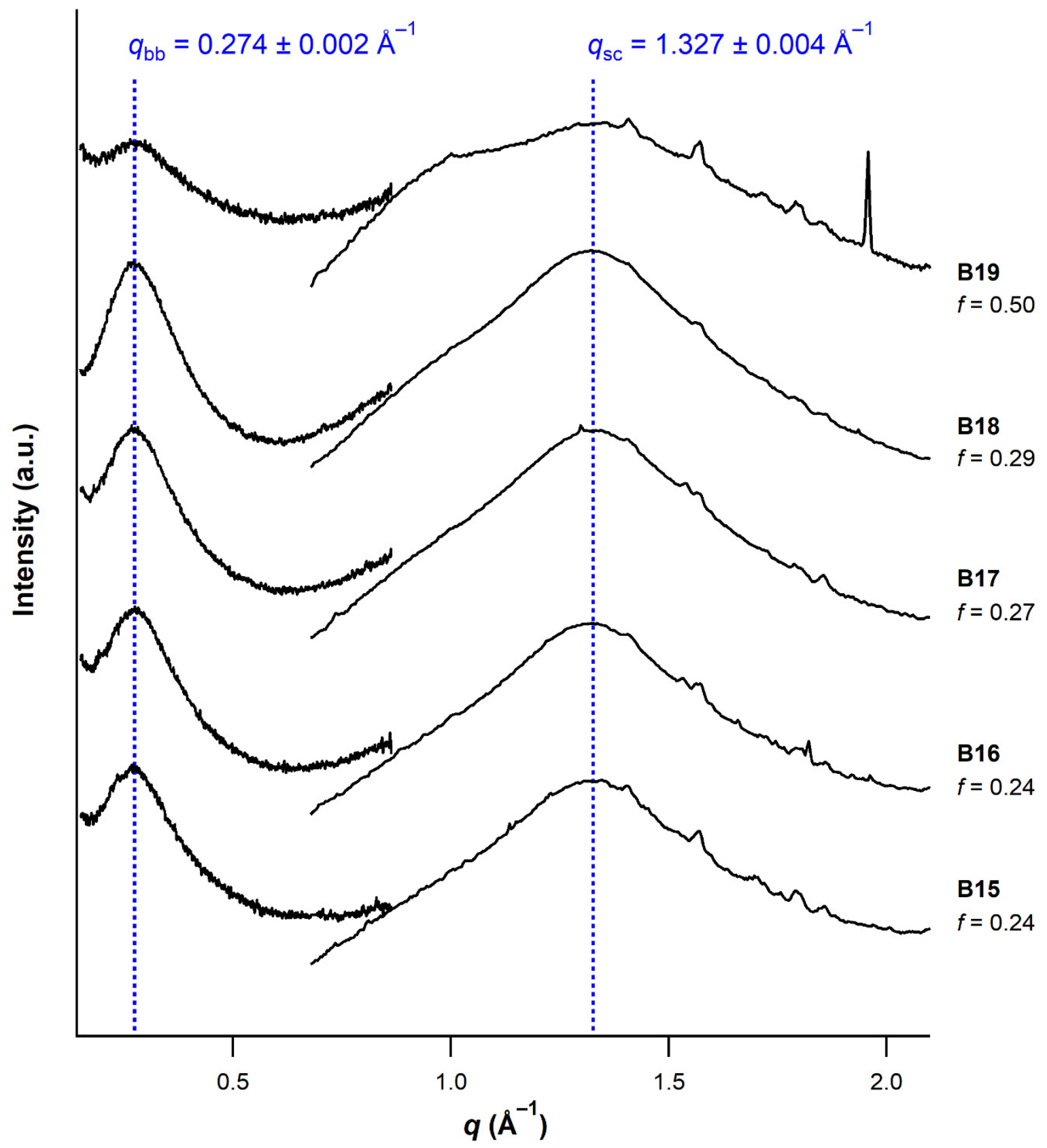

Figure S21. 1D-averaged mid- and wide-angle X-ray scattering (MAXS and WAXS) data for select PN2M- $b$-PN1D block polymers at $25^{\circ} \mathrm{C}$. Molecular characterization data for all samples is provided in Table S2. In the MAXS range, $q \mathrm{bb}=0.274 \pm 0.002 \AA^{-1}$. In the WAXS range, $q_{\mathrm{sc}}=1.327 \pm 0.004 \AA^{-1}$ corresponds to the average distance between $n$-decyl side chains. 
A. no side chain interdigitation

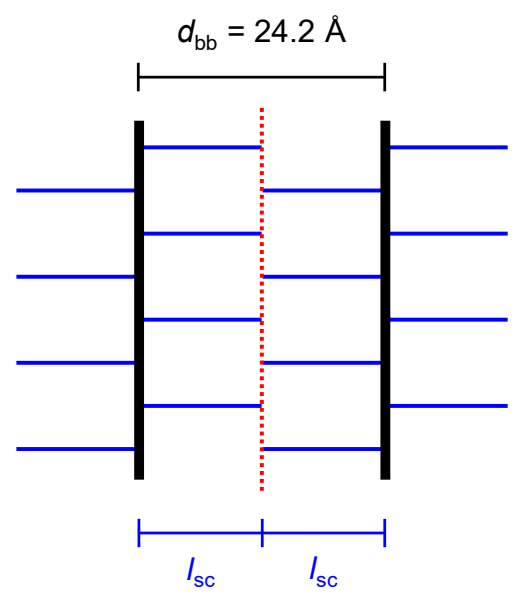

$$
\Rightarrow I_{\mathrm{sc}}=d_{\mathrm{bb}} / 2=12.1 \AA
$$

B. maximum side chain interdigitation

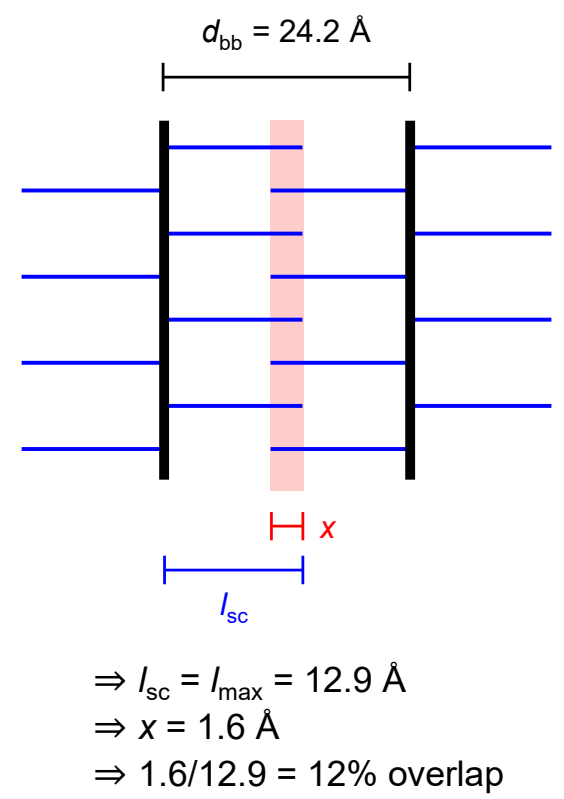

Figure S22. Schematic illustrations of the two limiting cases for estimating the side chain length $l_{\mathrm{sc}}$ from the backbone length $d_{\mathrm{bb}}$ (inferred from MAXS). (A) If no intermolecular overlap (i.e., no side chain interdigitation) occurs, $l_{\mathrm{sc}}=d_{\mathrm{bb}} / 2$. (B) If maximum overlap (i.e., maximum side chain interdigitation) occurs, $l_{\mathrm{sc}}=l_{\text {max. }}$. Values for PN2D are shown, where $d_{\mathrm{bb}}=24.2 \AA$ and $l_{\max }=12.9 \AA$, as calculated from Eq. 2. In (B), combining maximum extension and maximum interdigitation suggests that up to $12 \%$ overlap is possible.

\section{Estimation of statistical segment lengths by oscillatory shear rheology}

Statistical segment lengths $(b)$ were estimated for PN2M, PN2B, PN1D, and PN2D homopolymers from the plateau modulus $\left(G_{\mathrm{N}}\right){ }^{4-6} G_{\mathrm{N}}$ was determined for each homopolymer by oscillatory shear rheology as described in the Methods section. Note that the below derivation assumes Gaussian chain statistics, which (as discussed in the main text) may not be strictly valid for the polynorbornene polymers reported herein.

According to the equipartition theorem for $G_{\mathrm{N}}$, the molecular weight between entanglements $\left(M_{\mathrm{e}}\right)$ can be defined as follows:

$$
M_{e} \equiv \frac{\rho k N_{A v} T}{G_{N}}
$$

where $\rho$ is the density, $k$ is the Boltzmann constant, $N_{\mathrm{Av}}$ is the Avogadro constant, and $T$ is the temperature.

We then introduce the packing length $\left(p^{*}\right)$, which represents the ratio of the volume occupied by a chain to its mean-square end-to-end distance, ${ }^{4}$

$$
p^{*} \equiv \frac{M}{\rho N_{\mathrm{Av}}} \frac{1}{\left\langle R^{2}\right\rangle_{0}}
$$


where $M$ is the total molecular weight of the chain and $\left\langle R^{2}\right\rangle_{0}=N b^{2}$ is the mean-square end-to-end distance. Eq. S2 can be expressed in terms of the statistical segment length $(b)$ as

$$
p^{*}=\frac{M}{\rho N_{A v} N b^{2}}=\frac{m}{\rho N_{A v} b^{2}}
$$

where $m$ is the molecular weight per repeat unit. The molecular weight between entanglements can be expressed in terms of $p^{*}$ as

$$
M_{e}=\frac{\rho N_{A v}\left(p^{*}\right)^{3}}{\left(A^{\prime}\right)^{2}}
$$

where $A^{\prime}=0.0595$ is an empirically determined constant. (A detailed derivation can be found in Ref. 5, Ch. 11.6.2.)

Substituting Eqs. S3 into Eq. S4 and solving for $b$ obtains

$$
b=\frac{m^{1 / 2}}{M_{e}^{1 / 6}\left(A^{\prime} \rho N_{A v}\right)^{1 / 3}}
$$

For comparison across different systems, $b$ should be normalized to a common reference volume. Here, we use the arbitrary reference volume $v_{\text {ref }}=118 \AA^{3}$ to calculate normalized statistical segment lengths, $b_{\text {ref, }}$

$$
b_{\mathrm{ref}}=b\left(\frac{v_{\mathrm{ref}}}{v}\right)^{1 / 2}
$$

where $v=m / \rho N_{\mathrm{Av}}$ is the repeat unit volume.

These concepts can be used to calculate the self-concentration crossover parameter $\bar{N}_{\mathrm{x}}$, defined as the invariant entanglement degree of polymerization,

$$
\bar{N}_{\mathrm{x}}=\frac{N_{\mathrm{e}} b_{\mathrm{ref}}{ }^{6}}{v_{\mathrm{ref}}{ }^{2}}=\frac{M_{\mathrm{e}} b_{\mathrm{ref}}^{6}}{\rho N_{\mathrm{Av}} v_{\mathrm{ref}}{ }^{3}}
$$

where $N_{\mathrm{e}}=M_{\mathrm{e}} / \rho N_{\mathrm{Av}} v_{\text {ref }}$ is the normalized entanglement degree of polymerization. 


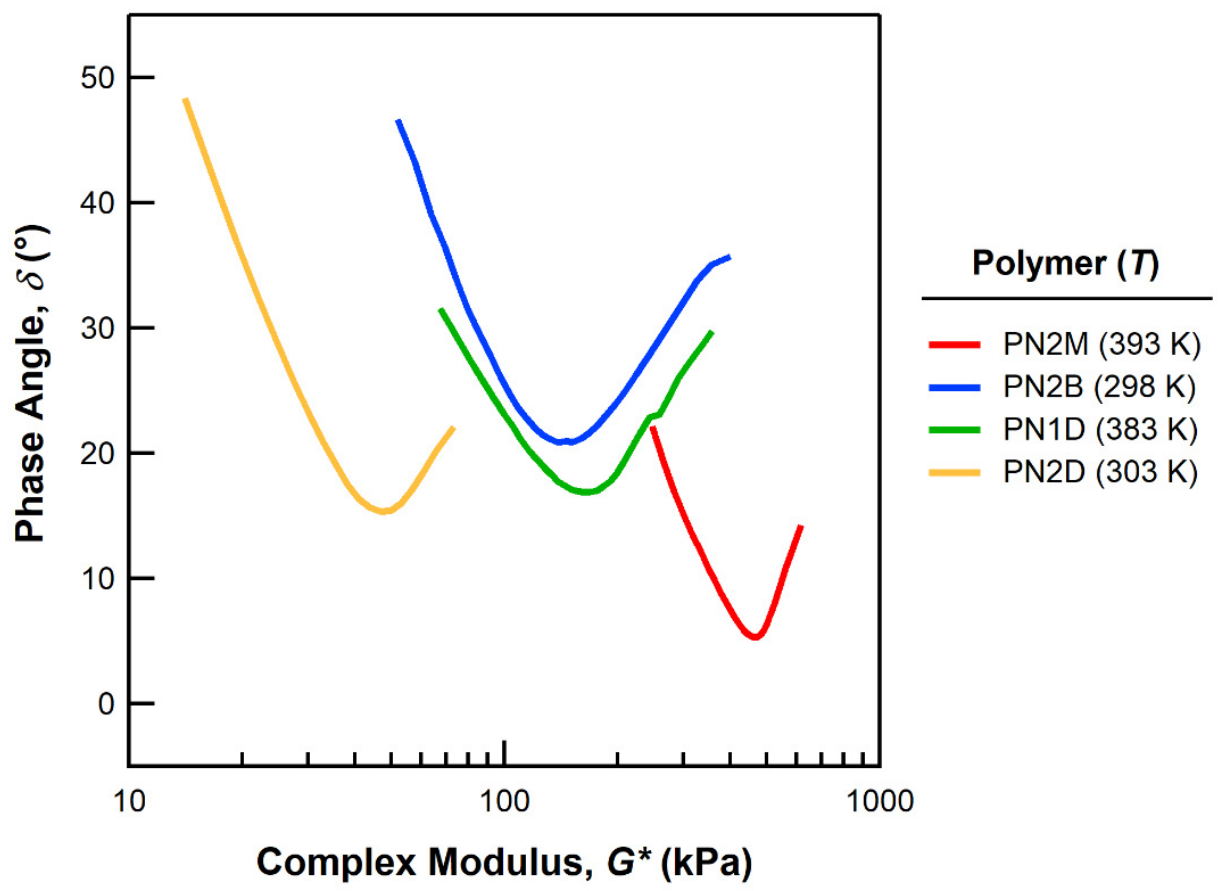

Figure S23. van Gurp-Palmen plots for the homopolymers poly(norbornene exo,exo-dimethylester) (PN2M, red), poly(norbornene exo,exo-di-n-butylester) (PN2B, blue), poly(norbornene exo- $n$-decylester) (PN1D, green), and poly(norbornene exo,exo-di- $n$-decylester) (PN2D, yellow). The temperatures $T$ at which the data were collected are provided in the legend. The plateau modulus $G_{\mathrm{N}}$ can be identified as the value of the complex modulus $G^{*}$ at the minimum in the phase angle $\delta$.

Table S8. Values used to calculate the entanglement molecular weight $\left(M_{\mathrm{e}}\right)$, normalized statistical segment length $\left(b_{\mathrm{ref}}\right)$, normalized entanglement degree of polymerization $\left(N_{\mathrm{e}}\right)$, and self-concentration crossover parameter $\left(\overline{\boldsymbol{N}}_{\mathbf{x}}\right)$.

\begin{tabular}{c|ccccccc} 
Polymer & $\begin{array}{c}\boldsymbol{\rho}^{\mathbf{a}} \\
\left(\mathbf{g} / \mathbf{c m}^{\mathbf{3}}\right)\end{array}$ & $\begin{array}{c}\boldsymbol{G}_{\mathbf{N}}^{\mathbf{b}} \\
(\mathbf{k P a})\end{array}$ & $\begin{array}{c}\boldsymbol{T} \\
(\mathbf{K})\end{array}$ & $\begin{array}{c}\boldsymbol{M}_{\mathbf{e}} \\
(\mathbf{k D a})\end{array}$ & $\begin{array}{c}\boldsymbol{b}_{\text {ref }} \mathbf{c}^{\mathbf{(}} \\
(\AA)\end{array}$ & $\boldsymbol{N}_{\mathrm{e}}$ & $\overline{\boldsymbol{N}}_{\mathbf{x}}$ \\
\hline PN2M & 1.22 & 460 & 393 & 8.6 & 5.84 & 100 & 280 \\
PN2B & 1.02 & 140 & 298 & 17.9 & 5.01 & 250 & 280 \\
PN1D & 1.01 & 165 & 383 & 19.5 & 5.06 & 270 & 320 \\
PN2D & 0.94 & 49 & 303 & 48.8 & 4.19 & 730 & 280
\end{tabular}

${ }^{a}$ Determined at room temperature using a density determination kit for Mettler-Toledo Excellence $\mathrm{XP} / \mathrm{XS}$ analytical balances.

${ }^{\text {b }}$ Determined by oscillatory shear rheology using $25 \mathrm{~mm}$ parallel plates at the temperature $T$ in the adjacent column.

${ }^{\mathbf{c}}$ Normalized to a common reference volume $v_{\text {ref }}=118 \AA^{3}$ according to Eq. S6. 


\section{References}

(1) Love, J. A.; Morgan, J. P.; Trnka, T. M.; Grubbs, R. H. A Practical and Highly Active Ruthenium-Based Catalyst that Effects the Cross Metathesis of Acrylonitrile. Angew. Chem. Int. Ed. 2002, 41, 4035-4037.

(2) Bates, C. M.; Chang, A. B.; Momčilović, N.; Jones, S. C.; Grubbs, R. H. ABA Triblock Brush Polymers: Synthesis, Self-Assembly, Conductivity, and Rheological Properties. Macromolecules 2015, 48, 4967.

(3) Chang, A. B.; Lin, T.-P.; Thompson, N. B.; Luo, S.-X.; Liberman-Martin, A. L.; Chen, H.-Y.; Lee, B.; Grubbs, R. H. Design, Synthesis, and Self-Assembly of Polymers with Tailored Graft Distributions. J. Am. Chem. Soc. 2017, 139, 17683.

(4) Fetters, L. J.; Lohse, D. J.; Richter, D.; Witten, T. A.; Zirkel, A. Connection between Polymer Molecular Weight, Density, Chain Dimensions, and Melt Viscoelastic Properties. Macromolecules 1994, 27, 46394647.

(5) Heimenz, P. C.; Lodge, T. P. Polymer Chemistry, 2nd ed.; CRC Press: Boca Raton, FL, 2007.

(6) Lewis, R. M.; Arora, A.; Beech, H. K.; Lee, B.; Lindsay, A. P.; Lodge, T. P.; Dorfman, K. D.; Bates, F. S. Role of Chain Length in the Formation of Frank-Kasper Phases in Diblock Copolymers. Phys. Rev. Lett. 2018, 121, 208002. 